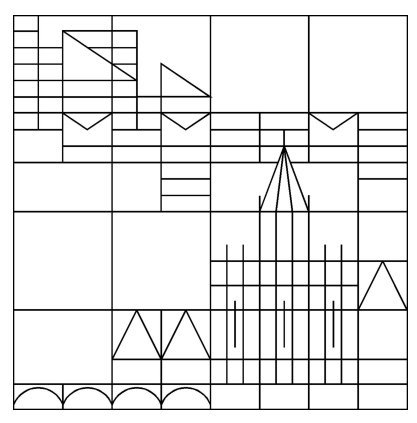

\title{
Estimates for solutions of a parameter-elliptic multi-order system of differential equations
}

\author{
Robert Denk \\ Melvin Faierman
}

Konstanzer Schriften in Mathematik

(vormals: Konstanzer Schriften in Mathematik und Informatik)

Nr. 257, September 2009

ISSN $1430-3558$

Konstanzer Online-Publikations-System (KOPS)

URN: http://nbn-resolving.de/urn:nbn:de:bsz:352-opus-86583

URL: http://kops.ub.uni-konstanz.de/volltexte/2009/8658/ 



\title{
ESTIMATES FOR SOLUTIONS OF A PARAMETER-ELLIPTIC MULTI-ORDER SYSTEM OF DIFFERENTIAL EQUATIONS
}

\author{
R. DENK AND M. FAIERMAN
}

\begin{abstract}
This paper is concerned with a boundary value problem defined over a bounded region of Euclidean space, and in particular it is devoted to the establishment of a priori estimates for solutions of a parameter-elliptic multiorder system of differential equations under limited smoothness assumptions. In this endeavour we extend the results of Agranovich, Denk, and Faierman pertaining to a priori estimates for solutions associated with a parameterelliptic scalar problem, as well as the results of various other authors who have extended the results of Agranovich et. al. from the scalar case to parameterelliptic systems of operators which are either of homogeneous type or have the property that the diagonal operators are all of the same order. In addition, we extend some results of Kozhevnikov and Denk and Volevich who have also dealt with sytems of the kind under consideration here, in that one of the works of Kozhevnikov deals only with $2 \times 2$ systems, while the other, as well as the work of the last two authors, do not cover Dirichlet boundary conditions.
\end{abstract}

\section{INTRODUCTION}

This paper is concerned with a boundary problem defined over a bounded region in $\mathbb{R}^{n}$, and in particular is devoted to the establishment of a priori estimates for solutions of a parameter-elliptic multi-order system of differential equations under limited smoothness assumptions. To elaborate on what was just said, let us now explain in more detail our two main objectives.

With regards to the first, we point out that very general results were derived in $[\mathrm{ADF}]$ pertaining to the spectral theory for scalar, non-selfadjoint elliptic boundary problems involving differential operators under limited smoothness assumptions and under a parameter-ellipticity condition. In particular, a method was developed there for deriving results pertaining to the eigenvalue asymptotics even under the limited conditions imposed. In [DFM] the techniques of $[\mathrm{ADF}]$ were used to extend the results for the scalar problem to that for a problem involving a parameter-elliptic system of differential operators of homogeneous type, and subsequently this result was extended in $[\mathrm{F}]$ to the case where only the diagonal operators of the system all had to be of the same order. It is important to mention at this point that all the spectral results derived in the above works depended fundamentally upon the establishment of a priori estimates for solutions of the boundary problem under consideration. Furthermore, in the above works, the a priori estimates could be established using standard methods, but when one attempts to extend the results of $[\mathrm{ADF}]$ to fully parameter-elliptic multi-order systems of differential operators,

Date: September 19, 2009.

1991 Mathematics Subject Classification. Primary 35J55; Secondary 35S15.

Key words and phrases. Parameter-ellipticity, multi-order systems, a priori estimates. 
one finds that the standard methods are no longer adequate, and new techniques must be introduced.

Accordingly, with all of this in mind, let us turn to the problem under consideration here. Let $N \in \mathbb{N}$ with $N>1$ and let $\left\{s_{j}\right\}_{1}^{N}$ and $\left\{t_{j}\right\}_{1}^{N}$ denote sequences of integers satisfying $s_{1} \geq s_{2} \geq \ldots \geq s_{N}, t_{1} \geq t_{2} \geq \ldots \geq t_{N} \geq 0$, and put $m_{j}=s_{j}+t_{j}$ for $j=1, \ldots, N$. We suppose that $m_{1}=m_{2}=\ldots=m_{k_{1}}>m_{k_{1}+1}=\ldots=m_{k_{d-1}}>$ $m_{k_{d-1}+1}=\ldots=m_{k_{d}}>0$, where $k_{d}=N$, put $\widetilde{m}_{j}=m_{k_{j}}$ for $j=1, \ldots, d$, and let $\widetilde{I}_{r}$ denote the $\left(k_{r}-k_{r-1}\right) \times\left(k_{r}-k_{r-1}\right)$ identity matrix for $r=1, \ldots, d$, where $k_{0}=0$. In the sequel we will use the notation $I_{\ell}$ to denote the $\ell \times \ell$ unit matrix for $\ell \in \mathbb{N}$ and also impose conditions which will ensure that for $r=1, \ldots, d$, the sum $\sum_{j=1}^{k_{r}} m_{j}$ is even; we henceforth denote this sum by $2 N_{r}$. Then with $\left\{\sigma_{j}\right\}_{1}^{\widetilde{N}}, \widetilde{N}=N_{d}$, denoting a sequence of integers such that $\max \left\{\sigma_{j}\right\}_{1}^{\widetilde{N}}<s_{N}$, we shall be concerned here with the boundary problem

$$
\begin{aligned}
A(x, D) u(x)-\lambda u(x) & =f(x) \text { in } \Omega, \\
B_{j}(x, D) u(x) & =g_{j}(x) \text { on } \Gamma \text { for } j=1, \ldots, \tilde{N},
\end{aligned}
$$

where $\Omega$ is a bounded region in $\mathbb{R}^{n}, n \geq 2$, with boundary $\Gamma, u(x)=\left(u_{1}(x), \ldots\right.$, $\left.u_{N}(x)\right)^{T}$, and $f(x)=\left(f_{1}(x), \ldots, f_{N}(x)\right)^{T}$ are $N \times 1$ matrix functions defined in $\Omega,{ }^{T}$ denotes transpose, the $g_{j}(x)$ are scalar functions defined on $\Gamma, A(x, D)$ is an $N \times N$ matrix operator whose entries $A_{j k}(x, D)$ are linear differential operators defined on $\Omega$ of order not exceeding $s_{j}+t_{k}$ and defined to be 0 if $s_{j}+t_{k}<0$, and $B_{j}(x, D), 1 \leq j \leq \widetilde{N}$, is a $1 \times N$ matrix operator whose entries $B_{j, k}(x, D)$ are linear differential operators defined on $\Gamma$ of order not exceeding $\sigma_{j}+t_{k}$, and defined to be 0 if $\sigma_{j}+t_{k}<0$. Our assumptions concerning the problem (1.1), (1.2) will be made precise in Section 2.

To motivate the second objective of this paper, let us point out that the first investigation into the spectral theory for a fully parameter-elliptic multi-order system of operators was instigated by Kozhevnikov [K1]. In this paper the author deals with a system of pseudodifferential operators acting over a compact manifold without boundary; and by introducing the so-called Kozhevnikov conditions, the author is able to establish a priori estimates for solutions as well as various other spectral results. In subsequent works [K2], [K3] the author deals with a genuine boundary problem under infinitely smooth conditions involving a parameter-elliptic multi-order system of differential operators acting over a bounded region in $\mathbb{R}^{n}$ and a system of differential operators defined on the boundary. In particular, in [K2] the author restricts himself to a $2 \times 2$ system of differential operators and a system of boundary operators which can be expressed in the form a a lower triangular block matrix, that is, in the terminology of this paper, we now have $N=$ $2, A(x, D)=\left(A_{j k}(x, D)\right)_{j, k=1}^{2}, B(x, D)=\left(B_{j k}(x, D)\right)_{\substack{j=1, \ldots, \mu / 2 \\ k=1,2}}=\left(T_{j k}(x, D)\right)_{j, k=1}^{2}$, with $T_{12}(x, D)=0$, where $\mu=\sum_{j=1}^{2}$ ord $A_{j j}(x, D)$. By introducing various conditions related to parameter-ellipticity, a result pertaining to the resolvent operator is established. In [K3] the author removes the restriction of [K2] that $N=2$, but now requires that the system of boundary operators admits a representation in the form of an upper triangular block matrix. Furthermore, he requires that $A(x, D)$ be of Petrovskii type. Then under various conditions, including those of [K1], the author derives some results pertaining to the resolvent operator. 
The problem considered in [K1] was also dealt with by Denk, Mennicken, and Volevich [DMV], but now in more detail. And by introducing conditions equivalent to those of Kozhevnikov and using the method of Newton polygons, the authors establish a priori estimates for solutions as well as various spectral results. In a subsequent work [DV], Denk and Volevich deal with a genuine boundary problem of the form (1.1), (1.2), but under the assumption that the operators $B_{j k}(x, D)$, if not identically zero, contain only top order terms and that these orders must satisfy a special condition. Then by appealing to the Kozhevnikov conditions as well as to the conditions of Vishik- Lyusternik [VL], the authors establish a priori estimates for solutions of the boundary problem (1.1), (1.2) for the case where $\Omega=\mathbb{R}_{+}^{n}$ and $\Gamma=\mathbb{R}^{n-1}$. What is novel in this latter work is the introduction of the Vishik-Lyusternik conditions, as this enables one to deal with multi-order systems of operators in the traditional way, that is, by means of contour integration.

Unfortunately, because of the restrictions imposed, both papers [K3] and [DV] are not even able to deal with the important problem where $s_{j}=t_{j}=t_{j}^{\prime}$ for $j=1, \ldots, N$, (here $\left\{t_{j}^{\prime}\right\}_{1}^{N}$ denotes a monotonic decreasing sequence of positive integers) and the boundary conditions are of Dirichlet type (see [ADN, Section 2], $[\mathrm{G}, \mathrm{p} .448])$. Of course the paper [K2] does include Dirichlet boundary conditions, but the requirement that $N=2$ is very restrictive.

Thus the first objective of this paper is to extend the results of [ADF] concerning solutions of the boundary problem for the scalar case to the non-scalar problem under consideration here, while our second objective is to extend the results of [K3] and $[\mathrm{DV}]$ by establishing a priori estimates for solutions of the boundary problem (1.1), (1.2) under boundary conditions which do include those of Dirichlet. And in this endeavour we shall make use of the conditions of both Kozhevnikov and VishikLyusternik (see Definitions 2.4 and 2.6 below). Our main result is then given in Theorem 2.8 below.

Let us also mention at this point that from a consideration of length, we have limited ourselves in this paper solely to the proof of the sufficiency part of Theorem 2.8 , that is to say, that the conditions cited in Definition 2.6 ensure the validity of this theorem. The converse of this result, that is, the proof of the necessity part of the theorem, will be left for a later work.

Finally, let us outline the contents of the paper. In Section 2 we introduce some terminology, definitions, and assumptions concerning the boundary problem (1.2), (1.2) which we require for our work and then present the main result of this work, namely Theorem 2.8 below. In Section 3 we restrict ourselves to the case where all the operators involved have constant coefficients and which act either over $\mathbb{R}^{n}$, without boundary conditions, or over $\mathbb{R}_{+}^{n}$, with boundary conditions defined on $\mathbb{R}^{n-1}$. All the preliminary results required for the proof of Theorem 2.8 are established here, with the main result being Proposition 3.6. These results are then used in Section 4 to prove Theorem 2.8.

\section{Preliminaries}

In this section we are going to introduce some terminology, definitions, and assumptions concerning the boundary problem (1.1), (1.2) which we require for our work and then state the main result of this paper, namely Theorem 2.8 below.

Accordingly, we let $x=\left(x_{1}, \ldots, x_{n}\right)=\left(x^{\prime}, x_{n}\right)$ denote a generic point in $\mathbb{R}^{n}$ and use the notation $D_{j}=-i \partial / \partial x_{j}, D=\left(D_{1}, \ldots D_{n}\right), D^{\alpha}=D_{1}^{\alpha_{1}} \cdots D_{n}^{\alpha_{n}}=D^{\prime \alpha^{\prime}} D_{n}^{\alpha_{n}}$, 
and $\xi=\xi_{1}^{\alpha_{1}} \ldots \xi_{n}^{\alpha_{n}}$ for $\xi=\left(\xi_{1}, \ldots, \xi_{n}\right)=\left(\xi^{\prime}, \xi_{n}\right) \in \mathbb{R}^{n}$, where $\alpha=\left(\alpha_{1}, \ldots, \alpha_{n}\right)=$ $\left(\alpha^{\prime}, \alpha_{n}\right)$ is a multi-index whose length $\sum_{j=1}^{n} \alpha_{j}$ is denoted by $|\alpha|$. Differentiation with respect to another variable, say $y \in \mathbb{R}^{n}$, instead of $x$ will be indicated by replacing $D, D^{\alpha}, D^{\prime \alpha^{\prime}}$, and $D_{n}^{\alpha_{n}}$ by $D_{y}, D_{y}^{\alpha}, D_{y^{\prime}}^{\prime \alpha^{\prime}}$, and $D_{y_{n}}^{\alpha_{n}}$, respectively. For $1<p<\infty, s \in \mathbb{N}_{0}=\mathbb{N} \cup\{0\}$, and $G$ an open set in $\mathbb{R}^{\ell}, \ell \in \mathbb{N}$, we let $W_{p}^{s}(G)$ denote the Sobolev space of order $s$ related to $L_{p}(G)$ and denote the norm in this space by $\|\cdot\|_{s, p, G}$, where $\|u\|_{s, p, G}=\left(\sum_{|\alpha| \leq s} \int_{G}\left|D^{\alpha} u(x)\right|^{p} d x\right)^{1 / p}$ for $u \in W_{p}^{s}(G)$. In addition we shall use norms depending upon a parameter $\lambda \in \mathbb{C} \backslash\{0\}$, namely for $1 \leq j \leq d$, we let

$$
\|u\|_{s, p, G}^{(j)}=\|u\|_{s, p, G}+|\lambda|^{s / \widetilde{m}_{j}}\|u\|_{0, p, G} \quad \text { for } u \in W_{p}^{s}(G) .
$$

We also let $\stackrel{\circ}{p}_{p}^{s}(G)$ denote the closure of $C_{0}^{\infty}(G)$ in $W_{p}^{s}(G)$.

In the sequel we shall also at times deal with the Bessel-potential space $H_{p}^{s}(G)$ for $0 \geq s \in \mathbb{Z}$ and equipped with norms depending upon the parameter $\lambda$. Namely for $u \in H_{p}^{s}(G)$ and $1 \leq j \leq d$, we introduce the norms $\|u\|_{s, p, G}^{(j)}=\left\|F^{-1}\langle\xi, \lambda\rangle_{j}^{s} F u\right\|_{0, p, \mathbb{R}^{n}}$ if $G=\mathbb{R}^{n}$ and $\|u\|_{s, p, G}^{(j)}=\inf \|v\|_{s, p, \mathbb{R}^{n}}^{(j)}$ otherwise, where the infimum is taken is taken over all $v \in H_{p}^{s}\left(\mathbb{R}^{n}\right)$ for which $u=v \mid G, F$ denotes the Fourier transformation in $\mathbb{R}^{n}(x \rightarrow \xi)$ and $\langle\xi, \lambda\rangle_{j}=\left(|\xi|^{2}+|\lambda|^{\frac{2}{m_{j}}}\right)^{\frac{1}{2}}$ (see [GK, Section 1], [T, p. 77]).

Assume for the moment that the boundary $\Gamma$ of $\Omega$ (see (1.1), (1.2)) is of class $C^{m-1,1}$ for some $m \in \mathbb{N}$, and let $s$ be an integer satisfying $1 \leq s \leq m$. Then for $G=\Omega \quad$ or $G=\mathbb{R}_{+}^{n}$, the vectors $u \in W_{p}^{s}(G)$ have boundary values $v=\left.u\right|_{\partial G}$ and we denote the space of these boundary values by $W_{p}^{s-1 / p}(\partial G)$ and denote by $\|\cdot\|_{s-1 / p, p, \partial G}$ the norm in this space, where $\|v\|_{s-1 / p, p, \partial G}=\inf \|u\|_{s, p, G}$ for $v \in W_{p}^{s-1 / p}(\partial G)$ and the infimum is taken over those $u \in W_{p}^{s}(G)$ for which $\left.u\right|_{\partial G}=v$ (see also [ADF, Section 2] and [Gr, p.20] for further definitions of $W_{p}^{s-1 / p}(\partial G)$ ). In addition we shall use norms depending upon a parameter $\lambda \in \mathbb{C} \backslash\{0\}$, namely for $1 \leq j \leq d$,

$$
\|v\|_{s-1 / p, p, \partial G}^{(j)}=\|v\|_{s-1 / p, p, \partial G}+|\lambda|^{(s-1 / p) / \widetilde{m}_{j}}\|v\|_{0, p, \partial G} \quad \text { for } v \in W_{p}^{s-1 / p}(\partial G),
$$

where $\|\cdot\|_{0, p, \partial G}$ denotes the norm in $L_{p}(\partial G)$.

Turning now to the boundary problem $(1.1),(1.2)$, let us write

$$
\begin{aligned}
& A_{j k}(x, D)=\sum_{|\alpha| \leq s_{j}+t_{k}} a_{\alpha}^{j k}(x) D^{\alpha} \quad \text { for } x \in \Omega \text { and } 1 \leq j, k \leq N, \\
& B_{j k}(x, D)=\sum_{|\alpha| \leq \sigma_{j}+t_{k}} b_{\alpha}^{j k}(x) D^{\alpha} \quad \text { for } x \in \Gamma \text { and } k=1, \ldots, N, j=1, \ldots, \tilde{N} .
\end{aligned}
$$

Observing that the orders of the operators $A_{j k}(x, D), B_{j k}(x, D)$ remain unchanged if we replace the sequences $\left\{s_{j}\right\}_{1}^{N},\left\{t_{j}\right\}_{1}^{N}$, and $\left\{\sigma_{j}\right\}_{1}^{\widetilde{N}}$ by $\left\{s_{j}-\sigma^{\dagger}\right\}_{1}^{N},\left\{t_{j}+\right.$ $\left.\sigma^{\dagger}\right\}_{1}^{N}$, and $\left\{\sigma_{j}-\sigma^{\dagger}\right\}_{1}^{\widetilde{N}}$, respectively, where $\sigma^{\dagger}=\max \left\{\sigma_{j}\right\}_{1}^{\widetilde{N}}+1$, we see that by making such substitutions if necessary, there is no loss of generality in henceforth supposing that $\sigma_{j}<0$ for $j=1, \ldots, \widetilde{N}$. Likewise, by replacing the sequences $\left\{s_{j}\right\}_{1}^{N},\left\{t_{j}\right\}_{1}^{N}$, and $\left\{\sigma_{j}\right\}_{1}^{\widetilde{N}}$ by $\left\{s_{j}-s_{N}\right\}_{1}^{N},\left\{t_{j}+s_{N}\right\}_{1}^{N}$, and $\left.\left\{\sigma_{j}-s_{N}\right\}_{1}^{\widetilde{N}}\right\}$, respectively, if necessary, we may also henceforth suppose that $s_{N} \geq 0$. 
Assumption 2.1. It will henceforth be supposed that $t_{j} \geq 0$ and $s_{j} \geq 0$ for $j=1, \ldots, N$, and that $\sigma_{j}<0$ for $j=1, \ldots, \widetilde{N}$.

Assumption 2.2. It will henceforth be supposed that: (1) $\Gamma$ is of class $C^{\kappa_{0}-1,1} \cap$ $C^{s_{1}}$, where $\kappa_{0}=\max \left\{t_{1}, \max \left\{-\sigma_{j}\right\}_{1}^{\widetilde{N}}\right\} ;(2)$ for each pair $j, k, a_{\alpha}^{j k} \in C^{s_{j}}(\bar{\Omega})$ for $|\alpha| \leq s_{j}+t_{k}$ if $s_{j}>0$, while if $s_{j}=0$, then $a_{\alpha}^{j k} \in L_{\infty}(\Omega)$ for $|\alpha|<s_{j}+t_{k}$ and $a_{\alpha}^{j k} \in C^{0}(\bar{\Omega})$ for $|\alpha|=s_{j}+t_{k}$; (3) for each pair $j, k, b_{\alpha}^{j k} \in C^{-\sigma_{j}-1,1}(\Gamma)$ for $|\alpha| \leq \sigma_{j}+t_{k}$.

For $\xi \in \mathbb{R}^{n}$ let

$$
\begin{gathered}
\stackrel{\circ}{A}(x, \xi)=\left(\stackrel{\circ}{A}_{j k}(x, \xi)\right)_{j, k}^{N} \text { for } x \in \bar{\Omega}, \\
\stackrel{\circ}{B}(x, \xi)=\left(\stackrel{\circ}{B}_{j k}(x, \xi)\right)_{\substack{j=1, \ldots, \widetilde{N} \\
k=1, \ldots, N}} \text { for } x \in \Gamma,
\end{gathered}
$$

and denote by $\stackrel{\circ}{B}_{j}(x, \xi)$ the $j$-th row of $\stackrel{\circ}{B}(x, \xi)$, where $\stackrel{\circ}{A}_{j k}(x, \xi)$ (resp. $\left.\stackrel{\circ}{B}_{j k}(x, \xi)\right)$ consists of those terms in $A_{j k}(x, \xi)$ (resp. $B_{j k}(x, \xi)$ ), which are just of order $s_{j}+t_{k}$ (resp. $\sigma_{j}+t_{k}$ ). Then in the sequel we shall also require the following notation. For $x \in \bar{\Omega}$ and $\xi \in \mathbb{R}^{n}$, let

$$
\begin{gathered}
\mathcal{A}_{11}^{(r)}(x, \xi)=\left(\AA_{j k}(x, \xi)\right)_{j, k=1}^{k_{r}} \text { for } 1 \leq r \leq d, \\
\mathcal{A}_{12}^{(r)}(x, \xi)=\left(\stackrel{\AA}{j k}_{j}(x, \xi)\right)_{\substack{j=1, \ldots, k_{r} \\
k=k_{r}+1, \ldots, N}}, \mathcal{A}_{21}^{(r)}(x, \xi)=\left(\AA_{j k}(x, \xi)\right)_{\substack{j=k_{r}+1, \ldots, N \\
k=1, \ldots, k_{r}}}, \text { and } \\
\mathcal{A}_{22}^{(r)}(x, \xi)=\left(\AA_{j k}(x, \xi)\right)_{j, k=k_{r}+1}^{N} \text { for } 1 \leq r \leq d-1 .
\end{gathered}
$$

Also for $x \in \Gamma, \xi \in \mathbb{R}^{n}$, and $1 \leq r \leq \ell_{1}, \ell \leq d$, let

$$
\begin{aligned}
& \mathcal{B}^{(r, \ell)}(x, \xi)=\left(\stackrel{\circ}{B}_{j k}(x, \xi)\right)_{\substack{j=N_{\ell-1}\left(1-\delta_{r, \ell}\right)+1, \ldots, N_{\ell} \\
k=1, \ldots, N}}, \\
& \mathcal{B}_{\ell_{1}, 1}^{(r, \ell)}(x, \xi)=\left(\stackrel{\circ}{B}_{j k}(x, \xi)\right)_{\substack{j=N_{\ell-1}\left(1-\delta_{r, \ell}\right)+1, \ldots, N_{\ell} \\
k=1, \ldots, k_{\ell}}},
\end{aligned}
$$

and

$$
\mathcal{B}_{\ell_{1}, 2}^{(r, \ell)}(x, \xi)=\left(\stackrel{\circ}{B}_{j k}(x, \xi)\right)_{\substack{j=N_{\ell-1}\left(1-\delta_{r, \ell}\right)+1, \ldots, N_{\ell} \\ k=k_{\ell_{1}}+1, \ldots, N}},
$$

where $\delta_{r, \ell}$ is the Kronecker delta. In addition we let $\widetilde{I}_{r, 0}=\operatorname{diag}\left(0 \cdot \widetilde{I}_{1}, \ldots, 0 \cdot \widetilde{I}_{r-1}, \widetilde{I}_{r}\right)$ for $r=2, \ldots, d$ and $\widetilde{I}_{1,0}=\widetilde{I}_{1}$.

Note that when $x^{0} \in \Gamma$ we can rewrite the boundary problem (1.1), (1.2) in terms of a local coordinate system at $x^{0}$ wherein $x^{0} \rightarrow 0$ and $\nu \rightarrow e_{n}$, where $\nu$ denotes the exterior normal to $\Gamma$ at $x^{0}$ and $\left(e_{1}, \ldots, e_{n}\right)$ denotes the standard basis in $\mathbb{R}^{n}$. Then supposing that this has been done, we shall in the sequel be concerned with the boundary problem

$$
\begin{aligned}
\stackrel{\circ}{A}(0, D) u(x)-\lambda u(x) & =f(x) \text { for } x \in \mathbb{R}_{+}^{n}, \\
\stackrel{\circ}{B}_{j}(0, D) u(x) & =g_{j}\left(x^{\prime}\right) \text { at } x_{n}=0 \text { for } j=1, \ldots, \widetilde{N},
\end{aligned}
$$

and corresponding to this boundary problem we define the associated symbols $\stackrel{\circ}{B}(0, \xi), \stackrel{\circ}{B}_{j, k}(0, \xi), \stackrel{\circ}{A}_{j, k}(0, \xi), \mathcal{A}_{j, k}^{(r)}(0, \xi), \mathcal{B}^{(r, \ell)}(0, \xi)$, and $\mathcal{B}_{\ell_{1}, j}^{(r, \ell)}(0, \xi)$ in precisely the same way their analogues were defined in the original coordinate system. 
Definition 2.3. Let $\mathcal{L}$ be a closed sector in the complex plane with vertex at the origin. Then the operator $A(x, D)-\lambda I_{N}$ will be called parameter-elliptic in $\mathcal{L}$ if $\operatorname{det}\left(\mathcal{A}_{11}^{(r)}(x, \xi)-\lambda \widetilde{I}_{r, 0}\right) \neq 0$ for $x \in \bar{\Omega}, \xi \in \mathbb{R}^{n} \backslash\{0\}$, and $\lambda \in \mathcal{L}, r=1, \ldots, d$.

In the sequel we let $\mathbb{C}_{ \pm}=\{z \in \mathbb{C}, \operatorname{Im} z \gtrless 0\}$.

Definition 2.4. Suppose that the operator $A(x, D)-\lambda I_{N}$ is parameter-elliptic in the sector $\mathcal{L}$ introduced above. Let $x^{0}$ be an arbitrary point of $\Gamma$ and assume that the boundary problem (1.1), (1.2) has been rewritten in a local coordinate system associated with $x^{0}$ in the manner just explained. Then the operator $A(x, D)-$ $\lambda I_{N}$ will be called properly parameter-elliptic in $\mathcal{L}$ if the following conditions are satisfied.

(1) The polynomial $\operatorname{det}\left(\mathcal{A}_{11}^{(r)}\left(0, \xi^{\prime}, z\right)-\lambda \widetilde{I}_{r, 0}\right)$ has precisely $N_{r}$ zeros lying in $\mathbb{C}_{+}$for $\xi^{\prime} \in \mathbb{R}^{n-1} \backslash\{0\}$ and $\lambda \in \mathcal{L}, r=1, \ldots, d$.

(2) The polynomial $\operatorname{det}\left(\mathcal{A}_{11}^{(r)}(0,0, z)-\lambda \widetilde{I}_{r, 0}\right)$ has precisely $N_{r}-N_{r-1}$ zeros lying in $\mathbb{C}_{+}$for $\lambda \in \mathcal{L} \backslash\{0\}, \quad r=2, \ldots, d$.

Remark 2.5. Referring to Condition (1) of Definition 2.4, we know from [AV, Section $2]$ that $\operatorname{det}\left(\mathcal{A}_{11}^{(r)}\left(0, \xi^{\prime}, z\right)-\lambda \widetilde{I}_{r, 0}\right)$ has precisely $N_{r}$ zeros in $\mathbb{C}_{+}$if $r=1$ or if $r>1$ and $n>2$. In the sequel, when proper parameter-ellipticity is supposed, it will be assumed that this is also the case when $r>1$ and $n=2$. Turning next to Condition (2) of the definition, it is clear that the number of zeros of the determinant in $\mathbb{C}_{+}$ (resp. $\mathbb{C}_{-}$) does not depend upon $\lambda$. Hence it follows from an expansion of the determinant in powers of $z$ and $\lambda$ that Condition (2) always holds if $\widetilde{m}_{r}$ is even or if $\widetilde{m}_{r}$ is odd, $k_{r}-k_{r-1}$ is even, and there is a $\lambda \in \mathcal{L} \backslash\{0\}$ such that $-\lambda \in \mathcal{L}$. Lastly we mention at this point that it is also clear from what was said above that Condition (2) is always satisfied if the operator $A(x, D)$ is essentially upper triangular at $x^{0}$ (see Definition 2.7 below)

Definition 2.6. Let $\mathcal{L}$ denote the sector introduced in Definition 2.3 above. Then we say that the boundary problem (1.1), (1.2) is parameter-elliptic in $\mathcal{L}$ if $A(x, D)-$ $\lambda I_{N}$ is properly parameter-elliptic in $\mathcal{L}$ and the following conditions are satisfied. Let $x^{0}$ be an arbitrary point of $\Gamma$ and suppose that the boundary problem (1.1), (1.2) has been rewritten in a local coordinate system associated with $x^{0}$, as explained above. Then

(1) the boundary problem on the half-line

$$
\begin{gathered}
\mathcal{A}_{11}^{(r)}\left(0, \xi^{\prime}, D_{n}\right) v\left(x_{n}\right)-\lambda \widetilde{I}_{r, 0} v\left(x_{n}\right)=0 \text { for } x_{n}>0, \\
\mathcal{B}_{r, 1}^{(r, r)}\left(0, \xi^{\prime}, D_{n}\right) v\left(x_{n}\right)=0 \text { at } x_{n}=0, \\
\left|v\left(x_{n}\right)\right| \rightarrow 0 \text { as } x_{n} \rightarrow \infty
\end{gathered}
$$

has only the trivial solution for $\xi^{\prime} \in \mathbb{R}^{n-1} \backslash\{0\}, \lambda \in \mathcal{L}$ and $1 \leq r \leq d$;

(2) the boundary problem on the half-line

$$
\begin{gathered}
\mathcal{A}_{11}^{(\ell)}\left(0,0, D_{n}\right) v\left(x_{n}\right)-\lambda \widetilde{I}_{\ell, 0} v\left(x_{n}\right)=0 \text { for } x_{n}>0, \\
\mathcal{B}_{\ell, 1}^{(r, \ell)}\left(0,0, D_{n}\right) v\left(x_{n}\right)=0 \text { at } x_{n}=0, \\
\left|v\left(x_{n}\right)\right| \rightarrow 0 \text { as } x_{n} \rightarrow \infty,
\end{gathered}
$$

has only the trivial solution for $\lambda \in \mathcal{L} \backslash\{0\}, 1 \leq r<d$ and $r<\ell \leq d$. 
For our purposes we need to introduce some further terminology. To this end we henceforth let $\pi_{1}(j)=r$ if $1 \leq j \leq N$ and $k_{r-1}<j \leq k_{r}$, and $\pi_{2}(j)=r$ if $1 \leq j \leq N_{d}$ and $N_{r-1}<j \leq N_{r}$, where $N_{0}=0$. In addition we let $\langle\xi\rangle=$ $\left(1+|\xi|^{2}\right)^{\frac{1}{2}},\left\langle\xi^{\prime}\right\rangle=\left(1+\left|\xi^{\prime}\right|^{2}\right)^{\frac{1}{2}}$, and $\left\langle\xi^{\prime}, \lambda\right\rangle_{j}=\left(\left|\xi^{\prime}\right|^{2}+|\lambda|^{\frac{2}{m_{j}}}\right)^{\frac{1}{2}}$ for $1 \leq j \leq d$. We also require the following definition.

Definition 2.7. Let $x^{0} \in \Gamma$. Then we say that the operator $A(x, D)$ is essentially upper triangular at $x^{0}$ if $a_{\alpha}^{j k}\left(x^{0}\right)=0$ for $|\alpha|=s_{j}+t_{k}, k_{\ell-1}<j \leq k_{\ell}, 1 \leq k \leq$ $k_{\ell-1}, \ell=2, \ldots, d$. Likewise we say that the operator $B(x, D)=\left(B_{j k}(x, D)\right)_{\substack{j=1, \ldots, \tilde{N} \\ k=1 \ldots, N}}$ is essentially upper triangular at $x^{0}$ if $b_{\alpha}^{j k}\left(x^{0}\right)=0$ for $|\alpha|=\sigma_{j}+t_{k}, N_{\ell-1}<j \leq$ $N_{\ell}, 1 \leq k \leq N_{\ell-1}, \ell=2, \ldots, d$.

We are now in a position to state the main result of this paper, namely Theorem 2.8 below, and which will be proved in Section 4. In this theorem we will require the further assumption, which will be made precise in Definition 3.13 below, that the operators $A(x, D)$ and $B(x, D)$ are compatible at each point of $\Gamma$. Hence for the moment let us state that this condition will always be satisfied if $B(x, D)$ is of Dirichlet type on $\Gamma$ or if the operators $A(x, D)$ and $B(x, D)$ are essentially upper triangular at every point of $\Gamma$.

Theorem 2.8. Suppose that the boundary problem (1.1), (1.2) is parameter-elliptic in $\mathcal{L}$. Suppose also that the operators $A(x, D)$ and $B(x, D)$ are compatible at every point of $\Gamma$. In addition, suppose that $B(x, D)$ is essentially upper triangular at every point of $\Gamma$. Then there exists a constant $\lambda^{0}=\lambda^{0}(p)>1$ such that for $\lambda \in \mathcal{L}$ with $|\lambda| \geq \lambda^{0}$, the boundary problem (1.1), (1.2) has a unique solution $u \in \prod_{j=1}^{N} W_{p}^{t_{j}}(\Omega)$ for every $f \in \prod_{j=1}^{N} H_{p}^{-s_{j}}(\Omega)$ and $g=\left(g_{1}, \ldots, g_{\widetilde{N}}\right)^{T} \in \prod_{j=1}^{\widetilde{N}} W_{p}^{-\sigma_{j}-\frac{1}{p}}(\Gamma)$, and the a priori estimate

$$
\sum_{j=1}^{N}\left\|u_{j}\right\|_{t_{j}, p, \Omega}\left(\pi_{1}(j)\right) \leq C\left(\sum_{j=1}^{N}\left\|f_{j}\right\|_{-s_{j}, p, \Omega}^{\left(\pi_{1}(j)\right)}+\sum_{j=1}^{\tilde{N}}\left\|g_{j}\right\|_{-\sigma_{j}-\frac{1}{p}, p, \Gamma}^{\left(\pi_{2}(j)\right)}\right)
$$

holds, where the constant $C$ does not depend upon the $f_{j}, g_{j}$, and $\lambda$.

Remark 2.9. The proof of Theorem 2.8 will depend upon the results of Section 3 and those of $[\mathrm{AV}]$, and as a consequence of these results it will also follow that the estimate (2.2) is 2 -sided, i.e., an estimate reverse to (2.2) holds. Indeed, we know from $[\mathrm{AV}]$ that we can cover $\bar{\Omega}$ by a finite number of open sets $\left\{U_{k}\right\}_{1}^{n_{1}}$, where $U_{k} \cap \Gamma \neq \emptyset$ if $k \leq n_{0}$ for some $n_{0}<n_{1}$, and $U_{k} \subset \Omega$ for $k>n_{0}$. If $\left\{\phi_{k}\right\}_{1}^{N_{1}}$ denotes a partition of unity subordinate to the covering $\left\{U_{k}\right\}_{1}^{n_{1}}$ such that $\operatorname{supp} \phi_{k} \cap \Gamma \neq \emptyset$ for $k \leq n_{0}$ and $\operatorname{supp} \phi_{k} \cap \Gamma=\emptyset$ otherwise, where supp denotes support, then a norm equivalent to the norm $\left\|f_{j}\right\|_{-s_{j}, p, \Omega}^{\left(\pi_{1}(j)\right)}$ defined above is given by

$$
\sum_{k=1}^{n_{0}}\left\|\phi_{k} f_{j}\right\|_{-s_{j}, p, \mathbb{R}_{+}^{n}}^{\left(\pi_{1}(j)\right)}+\sum_{k=n_{0}+1}^{n_{1}}\left\|\phi_{k} f_{j}\right\|_{-s_{j}, p, \mathbb{R}^{n}}^{\left(\pi_{1}(j)\right)}
$$

where the norm $\left\|\phi_{k} f_{j}\right\|_{-s_{j}, p, \mathbb{R}_{+}^{n}}^{\left(\pi_{1}(j)\right)}$ is taken in local coordinates. Since similar statements can be made for both $\left\|u_{j}\right\|_{t_{j}, p, \Omega}^{\left(\pi_{1}(j)\right)}$ and $\left\|g_{j}\right\|_{-\sigma_{j}-\frac{1}{p}, p, \Gamma}^{\left(\pi_{2}(j)\right)}$, the assertion concerning the 2-sidedness of (2.2) follows directly from the results of Section 3. 


\section{The Constant Coefficient Case}

In this section we are going to establish some results concerning the existence of and a priori estimates for solutions of a boundary problem involving constant coefficient systems which is related to (1.1), (1.2). These results will then be used in Section 4 to prove Theorem 2.8. To this end, let $x^{0} \in \Omega$ and let us fix our attention upon the differential equation

$$
\AA\left(x^{0}, D\right) u(x)-\lambda u(x)=f(x) \text { for } x \in \mathbb{R}^{n} \text { and } \lambda \in \mathcal{L} \backslash\{0\} .
$$

Then we have the following two results, where here and below we let $u_{j}$ and $f_{j}, 1 \leq$ $j \leq N$, denote the components of $u$ and $f$, respectively.

Proposition 3.1. Suppose that $u \in \prod_{j=1}^{N} W_{p}^{t_{j}}\left(\mathbb{R}^{n}\right)$ and that (3.1) holds. Then $f \in \prod_{j=1}^{N} H_{p}^{-s_{j}}\left(\mathbb{R}^{n}\right) \quad$ and $\quad \sum_{j=1}^{N}\left\|f_{j}\right\|_{-s_{j}, p, \mathbb{R}^{n}}^{\left(\pi_{1}(j)\right)} \leq C \sum_{j=1}^{N}\left\|u_{j}\right\|_{t_{j}, p, \mathbb{R}^{n}}^{\left(\pi_{1}(j)\right)}$, where the constant $C$ does not depend upon $u$ and $\lambda$.

Proposition 3.2. Suppose that the operator $A(x, D)-\lambda I_{N}$ is parameter-elliptic in $\mathcal{L}$ and that $f \in \prod_{j=1}^{N} H_{p}^{-s_{j}}\left(\mathbb{R}^{n}\right)$. Then there exists the constant $\lambda^{0}>0$ such that for $\lambda \in \mathcal{L}$ with $|\lambda| \geq \lambda^{0}$, the differential equation (3.1) has a unique solution $u \in$ $\prod_{j=1}^{N} W_{p}^{t_{j}}\left(\mathbb{R}^{n}\right) \quad$ and $\sum_{j=1}^{N}\left\|u_{j}\right\|_{t_{j}, p, \mathbb{R}^{n}}^{\left(\pi_{1}(j)\right)} \leq C \sum_{j=1}^{N}\left\|f_{j}\right\|_{-s_{j}, p, \mathbb{R}^{n}}^{\left(\pi_{1}(j)\right)}$, where the constant $C$ does not depend upon $f$ and $\lambda$.

We will only prove Proposition 3.2 as the proof of Proposition 3.1 follows directly from the definition and the Mikhlin-Lizorkin multiplier theorem.

Proof of Proposition 3.2. Under our assumptions we know from [DMV] and [K1] that for $\xi \in \mathbb{R}^{n}$ and $\lambda \in \mathcal{L}$ with $|\lambda| \geq \lambda^{0}, \AA\left(x^{0}, \xi\right)-\lambda I_{N}$ is invertible and

$$
\left|\operatorname{det}\left(\AA\left(x^{0}, \xi\right)-\lambda I_{N}\right)\right| \geq C \prod_{j=1}^{d}\langle\xi, \lambda\rangle_{j}^{2\left(N_{j}-N_{j-1}\right)},
$$

where the constant $C$ does not depend upon $\xi$ and $\lambda$. Furthermore, if we put $\left(\AA\left(x^{0}, \xi\right)-\lambda I_{N}\right)^{-1}=\left(\widetilde{a}_{j, k}(\xi, \lambda)\right)_{j, k=1}^{N}$, then the $\widetilde{a}_{j, k}(\xi, \lambda)$ are rational functions of their arguments, while it also follows from the references just cited that for any multi-index $\alpha$ whose entries are either 0 or 1 ,

$$
\left|\xi^{\alpha} D_{\xi}^{\alpha} \widetilde{a}_{j, k}(\xi, \lambda)\right| \leq C\langle\xi\rangle^{s_{j}+t_{k}}\langle\xi, \lambda\rangle_{\pi_{1}(j)}^{-m_{j}}\langle\xi, \lambda\rangle_{\pi_{1}(k)}^{-m_{k}}
$$

for all $\xi \in \mathbb{R}^{n}$ whose components are all non-zero, where the constant $C$ does not depend upon $\xi$ and $\lambda$.

Now observe that under a Fourier transformation (3.1) becomes

$$
\AA\left(x^{0}, \xi\right) F u(\xi)-\lambda F u(\xi)=F f(\xi) .
$$

Furthermore, in light of what was said above, we conclude that this equation has a unique solution in the space of tempered distributions on $\mathbb{R}^{n}$ given by $F u(\xi)=$ $\left(\AA\left(x^{0}, \xi\right)-\lambda I_{N}\right)^{-1} F f(\xi)$. Hence all of the assertions of the proposition follow immediately from this last result, the definitions of the terms involved, and the Mikhlin-Lizorkin multiplier theorem.

Let us suppose from now on that $x^{0} \in \Gamma$. Then assuming that the boundary problem (1.1), (1.2) has been rewritten in terms of the local coordinates at $x^{0}$ as 
explained in the text preceding Definition 2.3, let us fix our attention upon the problem in the half-space

$$
\AA(0, D) u(x)-\lambda u(x)=f(x) \text { for } x \in \mathbb{R}_{+}^{n} \text { and } \lambda \in \mathcal{L} \backslash\{0\} .
$$

Then from a consideration of the pairing between $H_{p}^{-s_{j}}\left(\mathbb{R}_{+}^{n}\right)$, equipped with the norm $\|\cdot\|_{-s_{j}, p, \mathbb{R}_{+}^{n}}^{\left(\pi_{1}(j)\right)}$, and its dual ${\stackrel{\circ}{W^{\prime}}}_{p_{j}}^{s_{j}}\left(\mathbb{R}_{+}^{n}\right)$, equipped with the norm $\|\cdot\|_{s_{j}, p^{\prime}, \mathbb{R}_{+}^{n}}^{\left(\pi_{1}(j)\right.}, 1 \leq$ $j \leq d, p^{\prime}=p /(p-1)$ (see [GK, Theorem 1.1]), we can easily derive the following analogue of Proposition 3.1.

Proposition 3.3. Suppose that $u \in \prod_{j=1}^{N} W_{p}^{t_{j}}\left(\mathbb{R}_{+}^{n}\right)$ and that (3.2) holds. Then $f \in \prod_{j=1}^{N} H_{p}^{-s_{j}}\left(\mathbb{R}_{+}^{n}\right) \quad$ and $\quad \sum_{j=1}^{N}\left\|f_{j}\right\|_{-s_{j}, p, \mathbb{R}_{+}^{n}}^{\left(\pi_{1}(j)\right)} \leq C \sum_{j=1}^{N}\left\|u_{j}\right\|_{t_{j}, p, \mathbb{R}_{+}^{n}}^{\left(\pi_{1}(j)\right)}$, where the constant $C$ does not depend upon $u$ and $\lambda$.

Proposition 3.4. Suppose that the operator $A(x, D)-\lambda I_{N}$ is parameter-elliptic in $\mathcal{L}$ and that $f \in \prod_{j=1}^{N} H_{p}^{-s_{j}}\left(\mathbb{R}_{+}^{n}\right)$. Then there exists a $\lambda^{0}>0$ such that for $\lambda \in \mathcal{L}$ with $|\lambda| \geq \lambda^{0}$, the differential equation (3.2) has a solution $u \in \prod_{j=1}^{N} W_{p}^{t_{j}}\left(\mathbb{R}_{+}^{n}\right)$ and $\sum_{j=1}^{N}\left\|u_{j}\right\|_{t_{j}, p, \mathbb{R}_{+}^{n}}^{\left(\pi_{1}(j)\right)} \leq C \sum_{j=1}^{N}\left\|f_{j}\right\|_{-s_{j}, p, \mathbb{R}_{+}^{n}}^{\left(\pi_{1}(j)\right)}$, where the constant $C$ does not depend upon $f$ and $\lambda$.

Proof. It follows from [T, Lemma 2.9.3, p.218] and [GK, Eqn.(1.27)] that there is a $\widetilde{f} \in \prod_{j=1}^{N} H_{p}^{-s_{j}}\left(\mathbb{R}^{n}\right)$ such that $\widetilde{f} \mid \mathbb{R}_{+}^{n}=f$ and

$$
\sum_{j=1}^{N}\left\|\widetilde{f}_{j}\right\|_{-s_{j}, p, \mathbb{R}^{n}}^{\left(\pi_{1}(j)\right)} \leq C \sum_{j=1}^{N}\left\|f_{j}\right\|_{-s_{j}, p, \mathbb{R}_{+}^{n}}^{\left(\pi_{1}(j)\right)},
$$

where the constant $C$ does not depend upon $f$ and $\lambda$. Hence if $\widetilde{u}$ denotes the solution of (3.1) when $f$ there is replaced by $\widetilde{f}$ and $u=\widetilde{u} \mid \mathbb{R}_{+}^{n}$, then the assertion of the proposition follows directly from Proposition 3.2.

Let us next fix our attention upon the boundary problem

$$
\begin{aligned}
\stackrel{\circ}{A}(0, D) u(x)-\lambda u(x) & =0 \text { for } x \in \mathbb{R}_{+}^{n}, \\
\stackrel{\circ}{B}_{j}(0, D) u(x) & =g_{j}\left(x^{\prime}\right) \text { at } x_{n}=0, j=1, \ldots, \widetilde{N},
\end{aligned}
$$

with $\lambda \in \mathbb{C} \backslash\{0\}$.

Proposition 3.5. Suppose that $u \in \prod_{j=1}^{N} W_{p}^{t_{j}}\left(\mathbb{R}_{+}^{n}\right)$ and that (3.4) holds. Then $g=\left(g_{1}, \ldots, g_{\tilde{N}}\right)^{T} \in \prod_{j=1}^{\tilde{N}} W_{p}^{-\sigma_{j}-\frac{1}{p}}\left(\mathbb{R}^{n-1}\right)$ and

$$
\sum_{j=1}^{\tilde{N}}\left\|g_{j}\right\|_{-\sigma_{j}-\frac{1}{p}, p, \mathbb{R}^{n-1}}^{\left(\pi_{2}(j)\right)} \leq C \sum_{j=1}^{N} \sum_{k=1}^{N}\left\|u_{k}\right\|_{t_{k}, p, \mathbb{R}_{+}^{n}}^{\left(\pi_{1}(j)\right.},
$$

where the constant $C$ does not depend upon $u$ and $\lambda$. Furthermore, if $B(x, D)$ is essentially upper triangular at $x^{0}$, then we may replace (3.5) by

$$
\sum_{j=1}^{\tilde{N}}\left\|g_{j}\right\|_{-\sigma_{j}-\frac{1}{p}, p, \mathbb{R}^{n-1}}^{\left(\pi_{2}(j)\right)} \leq C \sum_{j=1}^{N}\left\|u_{j}\right\|_{t_{j}, p, \mathbb{R}_{+}^{n}}^{\left(\pi_{1}(j)\right)}
$$


Proof. Let $1 \leq j \leq \widetilde{N}$ and let $\mu(j)=\pi_{2}(j)$ if $B(x, D)$ is essentially upper triangular at $x^{0}$ and let $\mu(j)=1$ otherwise. Then it follows from [ADF, Proposition 2.2] that

$$
\begin{aligned}
\left\|g_{j}\right\|_{-\sigma_{j}-\frac{1}{p}, p, \mathbb{R}^{n-1}}^{\left(\pi_{2}(j)\right)} & \leq\left. C_{1}\left\|\sum_{k=1}^{N} \sum_{|\alpha|=\sigma_{j}+t_{k}} b_{\alpha}^{j k}\left(x^{0}\right) D^{\alpha} u_{k}\right\|\right|_{-\sigma_{j}, p, \mathbb{R}_{+}^{n}} ^{\left(\pi_{2}(j)\right)} \\
& \leq C_{2} \sum_{k=\mu(j)}^{N} \sum_{|\alpha|=\sigma_{j}+t_{k}}\left(\left\|D^{\alpha} u_{k}\right\|_{-\sigma_{j}, p, \mathbb{R}_{+}^{n}}+|\lambda|^{\frac{-\sigma_{j}}{\bar{m}_{\pi_{2}(j)}}}\left\|D^{\alpha} u_{k}\right\|_{0, p, \mathbb{R}_{+}^{n}}\right) \\
& \leq C_{3} \sum_{k=\mu(j)}^{N}\left(\left\|u_{k}\right\|_{t_{k}, p, \mathbb{R}_{+}^{n}}+\left\|u_{k}\right\|_{t_{k}, p, \mathbb{R}_{+}^{n}}^{\left(\pi_{2}(j)\right)}\right) \\
& \leq 2 C_{3} \sum_{k=\mu(j)}^{N}\left\|u_{k}\right\|_{t_{k}, p, \mathbb{R}^{n}}^{\left(\pi_{2}(j)\right)}
\end{aligned}
$$

where the constants $C_{j}$ do not depend upon $u$ and $\lambda$. Hence all the assertions of the proposition follow from this last result.

We now come to the main result of this section.

Proposition 3.6. Suppose that the boundary problem (1.1), (1.2) is parameterelliptic in $\mathcal{L}$. Suppose also that the operators $A(x, D)$ and $B(x, D)$ are compatible at $x^{0}$. Then there exists a constant $\lambda^{0}=\lambda^{0}(p)>1$ such that for $\lambda \in \mathcal{L}$ with $|\lambda| \geq \lambda^{0}$, the boundary problem (3.3), (3.4) has a unique solution $u \in \prod_{j=1}^{N} W_{p}^{t_{j}}\left(\mathbb{R}_{+}^{n}\right)$ for every $g=\left(g_{1}, \ldots, g_{\widetilde{N}}\right)^{T} \in \prod_{j=1}^{\widetilde{N}} W_{p}^{-\sigma_{j}-\frac{1}{p}}\left(\mathbb{R}^{n-1}\right)$, and the a priori estimate

$$
\sum_{j=1}^{N}\left\|u_{j}\right\|_{t_{j}, p, \mathbb{R}_{+}^{n}}^{\left(\pi_{1}(j)\right.} \leq C \sum_{j=1}^{\tilde{N}}\left\|g_{j}\right\|_{-\sigma_{j}-\frac{1}{p}, p, \mathbb{R}^{n-1}}^{\left(\pi_{2}(j)\right)}
$$

holds, where the constant $C$ does not depend upon the $g_{j}$ and $\lambda$.

As a consequence of Propositions 3.4, 3.5, and 3.6 as well as from a standard argument, we obtain the following result.

Proposition 3.7. Suppose that the hypotheses of Proposition 3.6 hold. Suppose also that the operator $B(x, D)$ is essentially upper triangular at $x^{0}$. Then the exists a constant $\lambda^{0}=\lambda^{0}(p)>1$ such that for $\lambda \in \mathcal{L}$ with $|\lambda| \geq \lambda^{0}$, the boundary problem (3.2), (3.4) has a unique solution $u \in \prod_{j=1}^{N} W_{p}^{t_{j}}\left(\mathbb{R}_{+}^{n}\right)$ for every $f \in \prod_{j=1}^{N} H_{p}^{-s_{j}}\left(\mathbb{R}_{+}^{n}\right)$ and $g \in \prod_{j=1}^{\tilde{N}} W_{p}^{-\sigma_{j}-\frac{1}{p}}\left(\mathbb{R}^{n-1}\right)$, and the a priori estimate

$$
\sum_{j=1}^{N}\left\|u_{j}\right\|_{t_{j}, p, \mathbb{R}_{+}^{n}}^{\left(\pi_{1}(j)\right)} \leq C\left(\sum_{j=1}^{N}\left\|f_{j}\right\|_{-s_{j}, p, \mathbb{R}_{+}^{n}}^{\left(\pi_{1}(j)\right)}+\sum_{j=1}^{\tilde{N}}\left\|g_{j}\right\|_{-\sigma_{j}-\frac{1}{p}, j, p, \mathbb{R}^{n-1}}^{\left(\pi_{2}(j)\right)}\right)
$$

holds, where the constant $C$ does not depend upon $f, g$, and $\lambda$.

In order to prove Proposition 3.6, some preliminary results are required. Accordingly, let us fix $\lambda \in \mathcal{L}$ with $|\lambda|>1$ sufficiently large and let $\left\{\epsilon_{j}\right\}_{1}^{d}$ denote a sequence of numbers satisfying $0<\epsilon_{j}<1, j=1, \ldots, d$ (the magnitudes of $\lambda$ and 
the $\epsilon_{j}$ will be specified below). Also for $r=0, \ldots, d$, let us introduce functions $\psi_{r} \in C^{\infty}\left(\mathbb{R}^{n-1}\right)$ satisfying $0 \leq \psi_{r}\left(\xi^{\prime}\right) \leq 1$,

$$
\begin{aligned}
& \operatorname{supp} \psi_{0} \in\left\{\xi^{\prime} \in \mathbb{R}^{n-1}:\left|\xi^{\prime}\right| \leq \frac{3}{4} \epsilon_{1}|\lambda|^{1 / \tilde{m}_{1}}\right\} \text { and } \psi_{0}\left(\xi^{\prime}\right)=1 \text { for }\left|\xi^{\prime}\right| \leq \frac{1}{4} \epsilon_{1}|\lambda|^{1 / \tilde{m}_{1}}, \\
& \operatorname{supp} \psi_{r} \in\left\{\xi^{\prime} \in \mathbb{R}^{n-1}: \frac{1}{4} \epsilon_{r}|\lambda|^{1 / \tilde{m}_{r}} \leq\left|\xi^{\prime}\right| \leq \frac{3}{4} \epsilon_{r+1}|\lambda|^{1 / \tilde{m}_{r+1}}\right\} \\
& \text { and } \psi_{r}\left(\xi^{\prime}\right)=1 \text { for } \frac{3}{4} \epsilon_{r}|\lambda|^{1 / \widetilde{m}_{r}} \leq\left|\xi^{\prime}\right| \leq \frac{1}{4} \epsilon_{r+1}|\lambda|^{1 / \widetilde{m}_{r+1}}, 1 \leq r<d, \\
& \operatorname{supp} \psi_{d} \in\left\{\xi^{\prime} \in \mathbb{R}^{n-1}:\left|\xi^{\prime}\right| \geq \frac{1}{4} \epsilon_{d}|\lambda|^{1 / \tilde{m}_{d}}\right\} \text { and } \psi_{d}\left(\xi^{\prime}\right)=1 \text { for }\left|\xi^{\prime}\right| \geq \frac{3}{4} \epsilon_{d}|\lambda|^{1 / \widetilde{m}_{d},},
\end{aligned}
$$

while in addition $\sum_{r=0}^{d} \psi_{r}\left(\xi^{\prime}\right)=1$ and each $\psi_{r}$ is a Fourier multiplier of type $(p, p)$ whose norm is bounded by a constant not depending upon $\lambda$ and the $\epsilon_{j}$. Then in the sequel we will require the following three results.

Proposition 3.8. Suppose that the boundary problem (1.1),(1.2) is parameterelliptic in $\mathcal{L}$. Suppose also that $\lambda \in \mathcal{L}$ with $|\lambda|>1$ and that $0 \leq\left|\xi^{\prime}\right| \leq \frac{7}{8} \epsilon_{1}|\lambda|^{\frac{1}{m_{1}}}$. Then we can choose the numbers $\epsilon^{0}$ sufficiently small and $\lambda^{0}$ sufficiently large so that for $\epsilon_{1} \leq \epsilon^{0}$ and $|\lambda| \geq \lambda^{0}$, $\operatorname{det}\left(\AA\left(0, \xi^{\prime}, z\right)-\lambda I_{N}\right)$ has precisely $N_{d}(=\widetilde{N})$ zeros, say $\left\{z_{j}^{(0)}\left(\xi^{\prime}, \lambda\right)\right\}_{j=1}^{N_{d}}$, lying in $\mathbb{C}_{+}$, and satisfying

$$
\begin{aligned}
& \operatorname{Im} z_{j}^{(0)}\left(\xi^{\prime}, \lambda\right) \geq C_{1}\left\langle\xi^{\prime}, \lambda\right\rangle_{1}, \quad\left|z_{j}^{(0)}\left(\xi^{\prime}, \lambda\right)\right| \leq C_{2}\left\langle\xi^{\prime}, \lambda\right\rangle_{1}, j=1, \ldots, N_{1}, \\
& \operatorname{Im} z_{j}^{(0)}\left(\xi^{\prime}, \lambda\right) \geq C_{1}\left\langle\xi^{\prime}, \lambda\right\rangle_{\ell}, \quad\left|z_{j}^{(0)}\left(\xi^{\prime}, \lambda\right)\right| \leq C_{2}\left\langle\xi^{\prime}, \lambda\right\rangle_{\ell}, j=N_{\ell-1}+1, \ldots, N_{\ell}
\end{aligned}
$$

for $\ell=2, \ldots, d$, and where $C_{2}\left\langle\xi^{\prime}, \lambda\right\rangle_{\ell}<C_{1}\left\langle\xi^{\prime}, \lambda\right\rangle_{\ell+1}$ for $\ell=1 \ldots, d-1$, and the $C_{j}$ denote constants not depending upon $\xi^{\prime}$ and $\lambda$.

Proposition 3.9. Suppose that the boundary problem (1.1), (1.2) is parameterelliptic in $\mathcal{L}$. Suppose also that $1 \leq r<d$, that $\lambda \in \mathcal{L}$ with $|\lambda|>1$, and that $\frac{1}{8} \epsilon_{r}|\lambda|^{\frac{1}{m_{r}}} \leq\left|\xi^{\prime}\right| \leq \frac{7}{8} \epsilon_{r+1}|\lambda|^{\frac{1}{m_{r+1}}}$. Then for fixed $\epsilon_{r}$ we can choose the numbers $\epsilon^{0}$ sufficiently small and $\lambda^{0}$ sufficiently large so that for $\epsilon_{r+1} \leq \epsilon^{0}$ and $|\lambda| \geq \lambda^{0}$, $\operatorname{det}\left(\AA\left(0, \xi^{\prime}, z\right)-\lambda I_{N}\right)$ has precisely $N_{d}$ zeros, say $\left\{z_{j}^{(r)}\left(\xi^{\prime}, \lambda\right)\right\}_{j=1}^{N_{d}}$, lying in $\mathbb{C}_{+}$ and satisfying

$$
\begin{array}{rlrl}
\operatorname{Im} z_{j}^{(r)}\left(\xi^{\prime}, \lambda\right) & \geq C_{1}\left\langle\xi^{\prime}, \lambda\right\rangle_{r}, & \left|z_{j}^{(r)}\left(\xi^{\prime}, \lambda\right)\right| \leq C_{2}\left\langle\xi^{\prime}, \lambda\right\rangle_{r}, j=1, \ldots, N_{r}, \\
\operatorname{Im} z_{j}^{(r)}\left(\xi^{\prime}, \lambda\right) \mid \geq C_{1}\left\langle\xi^{\prime}, \lambda\right\rangle_{\ell}, & \left|z_{j}^{(r)}\left(\xi^{\prime}, \lambda\right)\right| \leq C_{2}\left\langle\xi^{\prime}, \lambda\right\rangle_{\ell}, j=N_{\ell-1}, \ldots, N_{\ell}
\end{array}
$$

for $\ell=r+1, \ldots, d$, and where $C_{2}\left\langle\xi^{\prime}, \lambda\right\rangle_{\ell}<C_{1}\left\langle\xi^{\prime}, \lambda\right\rangle_{\ell+1}$ for $\ell=r, \ldots, d-1$, and the $C_{j}$ denote positive constants not depending upon $\xi^{\prime}$ and $\lambda$.

Proposition 3.10. Suppose that the boundary problem (1.1), (1.2) is parameterelliptic in $\mathcal{L}$. Suppose also that $\lambda \in \mathcal{L}$ with $|\lambda|>1$ and that $\frac{1}{8} \epsilon_{d}|\lambda|^{\frac{1}{m_{d}}} \leq\left|\xi^{\prime}\right|<\infty$. Then for fixed $\epsilon_{d}$ we can choose the number $\lambda^{0}$ sufficiently large so that for $|\lambda| \geq \lambda^{0}$, $\operatorname{det}\left(\AA\left(0, \xi^{\prime}, z\right)-\lambda I_{N}\right)$ has precisely $N_{d}$ zeros, say $\left\{z_{j}^{(d)}\left(\xi^{\prime}, \lambda\right)\right\}_{j=1}^{N_{d}}$, lying in $\mathbb{C}_{+}$and satisfying

$$
\operatorname{Im} z_{j}^{(d)}\left(\xi^{\prime}, \lambda\right) \geq C_{1}\left\langle\xi^{\prime}, \lambda\right\rangle_{d}, \quad\left|z_{j}^{(d)}\left(\xi^{\prime}, \lambda\right)\right| \leq C_{2}\left\langle\xi^{\prime}, \lambda\right\rangle_{d} \text { for } j=1, \ldots, N_{d},
$$

where the $C_{j}$ denote constants not depending upon $\xi^{\prime}$ and $\lambda$.

Since the proofs of Propositions 3.8 and 3.10 are similar to that of Proposition 3.9 , we will only prove this latter proposition. 
Proof of Proposition 3.9. To begin with let us observe that

$$
\AA\left(0, \xi^{\prime}, z\right)-\lambda I_{N}=\left(\begin{array}{cc}
\mathcal{A}_{11}^{(r)}\left(0, \xi^{\prime}, z\right)-\lambda I_{k_{r}} & \mathcal{A}_{12}^{(r)}\left(0, \xi^{\prime}, z\right) \\
\mathcal{A}_{21}^{(r)}\left(0, \xi^{\prime}, z\right) & \mathcal{A}_{22}^{(r)}\left(0, \xi^{\prime}, z\right)-\lambda I_{N-k_{r}}
\end{array}\right)
$$

and that

$$
\mathcal{A}_{11}^{(r)}\left(0, \xi^{\prime}, z\right)-\lambda I_{k_{r}}=\mathcal{A}_{11}^{(r)}\left(0, \xi^{\prime}, z\right)-\lambda \widetilde{I}_{r, 0}-\lambda\left(I_{k_{r}}-\widetilde{I}_{r, 0}\right)
$$

Then as a consequence of our hypotheses we know that $\operatorname{det}\left(\mathcal{A}_{11}^{(r)}\left(0, \xi^{\prime}, z\right)-\lambda \widetilde{I}_{r, 0}\right)$ has precisely $N_{r}$ zeros lying in $\mathbb{C}_{+}$and that there is a closed contour $\gamma_{r}^{+}\left(\xi^{\prime}, \lambda\right) \subset$ $\mathbb{C}_{+}$containing all these zeros in its interior such that for $z \in \gamma_{r}^{+}\left(\xi^{\prime}, \lambda\right), \operatorname{Im} z \geq$ $C_{1}\left\langle\xi^{\prime}, \lambda\right\rangle_{r},|z| \leq C_{2}\left\langle\xi^{\prime}, \lambda\right\rangle_{r}$, and

$$
C_{3}\left\langle\xi^{\prime}, \lambda\right\rangle_{r}^{2 N_{r}} \leq\left|\operatorname{det}\left(\mathcal{A}_{11}^{(r)}\left(0, \xi^{\prime}, z\right)-\lambda \widetilde{I}_{r, 0}\right)\right| \leq C_{4}\left\langle\xi^{\prime}, \lambda\right\rangle_{r}^{2 N_{r}},
$$

where the constants $C_{j}$ do not depend upon $\xi^{\prime}, z$, and $\lambda$. Furthermore, it is easy to show that for $z \in \gamma_{r}^{+}\left(\xi^{\prime}, \lambda\right)$,

$$
\begin{aligned}
& \left|\operatorname{det}\left(\mathcal{A}_{11}^{(r)}\left(0, \xi^{\prime}, z\right)-\lambda I_{k_{r}}\right)-\operatorname{det}\left(\mathcal{A}_{11}^{(r)}\left(0, \xi^{\prime}, z\right)-\lambda \widetilde{I}_{r, 0}\right)\right| \leq \\
& C \sum_{\ell=1}^{k_{r-1}} \sum_{1 \leq i(1)<\ldots<i(\ell) \leq k_{r-1}} \prod_{k=1}^{\ell}\left(\frac{|\lambda|^{1 / m_{i(k)}}}{\left\langle\xi^{\prime}, \lambda\right\rangle_{r}}\right)^{m_{i(k)}}\left\langle\xi^{\prime}, \lambda\right\rangle_{r}^{2 N_{r}},
\end{aligned}
$$

where the constant $C$ does not depend upon $\xi^{\prime}, z$, and $\lambda$, while by employing the Laplace method of expanding a determinant, we can also show that, apart from a constant not depending upon $\xi^{\prime}, z$, and $\lambda$, the term $\mid \operatorname{det}\left(\AA\left(0, \xi^{\prime}, z\right)-\lambda I_{N}\right)$ $\operatorname{det}\left(\mathcal{A}_{11}^{(r)}\left(0, \xi^{\prime}, z\right)-\lambda I_{k_{r}}\right) \lambda^{N-k_{r}} \mid$ is bounded by the sum

$$
\begin{aligned}
& \left|\operatorname{det}\left(\mathcal{A}_{11}^{(r)}\left(0, \xi^{\prime}, z\right)-\lambda I_{k_{r}}\right)\right| \sum_{\mu=0}^{N-k_{r}-1}\left(\left\langle\xi^{\prime}, z\right\rangle^{\widetilde{m}_{r+1}}\right)^{N-k_{r}-\mu}|\lambda|^{\mu}+ \\
& \sum_{q}\left(\left\langle\xi^{\prime}, z\right\rangle^{\sum_{\mu=1}^{q} m_{i_{\mu}}} \sum_{j_{1}<\ldots<j_{\nu}}\left\langle\xi^{\prime}, z\right\rangle^{\sum_{\mu=1}^{\nu} m_{j_{\mu}}|\lambda|^{k_{r}-q-\nu}}\right) \times \\
& \left(\left\langle\xi^{\prime}, z\right\rangle^{\sum_{\mu=1}^{q} m_{\tilde{i}_{\mu}}} \sum_{\tilde{j}_{1}<\ldots<\tilde{j}_{\nu}}\left\langle\xi^{\prime}, z\right\rangle^{\left.\sum_{\mu=1}^{\nu} m_{\tilde{j}_{\mu}}|\lambda|^{N-k_{r}-q-\nu}\right) .}\right.
\end{aligned}
$$

Here $\left\langle\xi^{\prime}, z\right\rangle=\left(\left|\xi^{\prime}\right|^{2}+|z|^{2}\right)^{\frac{1}{2}}, \sum_{q}$ indicates that the summation is over those $q$ satisfying $1 \leq q \leq k_{r}$ (resp. $1 \leq q \leq N-k_{r}$ ) if $2 k_{r} \leq N$ (resp. $2 k_{r}>N$ ), and $\left\{i_{\mu}\right\}_{\mu=1}^{q}$ and $\left\{j_{\mu}\right\}_{\mu=1}^{\nu}$ denote distinct sequences of integers satisfying $1 \leq i_{1}<\ldots<$ $i_{q} \leq k_{r}$ and $1 \leq j_{1}<\ldots<j_{\nu} \leq k_{r}, 1 \leq \nu \leq k_{r}-q$, respectively, while $\left\{\widetilde{i}_{\mu}\right\}_{\mu=1}^{q}$ and $\left\{\widetilde{j}_{\mu}\right\}_{\mu=1}^{\nu}$ denote distinct sequences of integers satisfying $k_{r}+1 \leq \widetilde{i}_{1}<\ldots<\widetilde{i}_{q} \leq N$ and $k_{r}+1 \leq \widetilde{j}_{1}<\ldots<\widetilde{j}_{\nu} \leq N, 1 \leq \nu \leq N-k_{r}-q$, respectively, and where the summation $\sum_{j_{1}<\ldots<j_{\nu}}$ in (3.6) is to be replaced by 1 if $q=k_{r}$ and the summation $\sum_{\widetilde{j}_{1}<\ldots<\tilde{j}_{\nu}}$ in (3.6) is to be replaced by 1 if $q=N-k_{r}$. Hence it follows from Rouché's theorem that if $\epsilon_{r+1}$ is chosen sufficiently small and $|\lambda|$ sufficiently large, then $\operatorname{det}\left(\AA\left(0, \xi^{\prime}, z\right)-\lambda I_{N}\right)$ has precisely $N_{r}$ zeros contained in $\gamma_{r}^{+}\left(\xi^{\prime}, \lambda\right)$. 
Suppose next that $\ell>r$. Then we can write

$$
\AA\left(0, \xi^{\prime}, z\right)-\lambda I_{N}=\left(\begin{array}{cc}
\mathcal{A}_{11}^{(\ell)}\left(0, \xi^{\prime}, z\right)-\lambda I_{k_{\ell}} & \mathcal{A}_{12}^{(\ell)}\left(0, \xi^{\prime}, z\right) \\
\mathcal{A}_{21}^{(\ell)}\left(0, \xi^{\prime}, z\right) & \mathcal{A}_{22}^{(\ell)}\left(0, \xi^{\prime}, z\right)-\lambda I_{N-k_{\ell}}
\end{array}\right)
$$

if $\ell<d$, and where (3.7) must be modified in an obvious way if $\ell=d$. In addition we have

$\mathcal{A}_{11}^{(\ell)}\left(0, \xi^{\prime}, z\right)-\lambda I_{k_{\ell}}=\mathcal{A}_{11}^{(\ell)}(0,0, z)-\lambda \widetilde{I}_{\ell, 0}+\mathcal{A}_{11}^{(\ell)}\left(0, \xi^{\prime}, z\right)-\mathcal{A}_{11}(0,0, z)-\lambda\left(I_{k_{\ell}}-\widetilde{I}_{\ell, 0}\right)$.

Then as a consequence of our hypotheses we know that $\operatorname{det}\left(\mathcal{A}_{11}^{(\ell)}(0,0, z)-\lambda \widetilde{I}_{\ell, 0}\right)$ has precisely $N_{\ell}-N_{\ell-1}$ zeros lying in $\mathbb{C}_{+}$and that there is a closed contour $\gamma_{r, \ell}^{+}(\lambda) \subset$ $\mathbb{C}_{+}$containing all these zeros in its interior such that for $z \in \gamma_{r, \ell}^{+}(\lambda), \operatorname{Im} z \geq$ $C_{1}^{\prime}|\lambda|^{\frac{1}{\bar{m}_{\ell}}},|z| \leq C_{2}^{\prime}|\lambda|^{\frac{1}{\bar{m}_{\ell}}}$ and $C_{3}^{\prime}|\lambda|^{\frac{2 N_{\ell}}{\bar{m}_{\ell}}} \leq\left|\operatorname{det}\left(\mathcal{A}_{11}^{(\ell)}(0,0, z)-\lambda \widetilde{I}_{\ell, 0}\right)\right| \leq C_{4}^{\prime}|\lambda|^{\frac{2 N_{\ell}}{\bar{m}_{\ell}}}$, where the constants $C_{j}^{\prime}$ do not depend upon $\lambda$. Furthermore, we can show that for $z \in \gamma_{r, \ell}^{+}(\lambda)$,

$$
\begin{aligned}
& \left|\operatorname{det}\left(\mathcal{A}_{11}^{(\ell)}\left(0, \xi^{\prime}, z\right)-\lambda I_{k_{\ell}}\right)-\operatorname{det}\left(\mathcal{A}_{11}^{(\ell)}(0,0, z)-\lambda \widetilde{I}_{\ell, 0}\right)\right| \\
& \leq C\left(|\lambda|^{\frac{1}{\bar{m}_{\ell-1}}-\frac{1}{\bar{m}_{\ell}}}+\delta_{\ell, r+1} \epsilon_{r+1}\right)|\lambda|^{\frac{2 N_{\ell}}{\bar{m}_{\ell}}}
\end{aligned}
$$

where $\delta_{\ell, r+1}$ denotes the Kronecker delta and the constant $C$ does not depend upon $\xi^{\prime}, z$, and $\lambda$, and that, apart from a constant not depending upon $\xi^{\prime}, z$ and $\lambda$, $\left|\operatorname{det}\left(\AA\left(0, \xi^{\prime}, z\right)-\lambda I_{N}\right)-\operatorname{det}\left(\mathcal{A}_{11}^{(\ell)}\left(0, \xi^{\prime}, z\right)-\lambda I_{k_{\ell}}\right) \lambda^{N-k_{\ell}}\right|$ is bounded by the expression (3.6) if we replace $r$ there by $\ell$. It follows again from Rouché's theorem that if $\epsilon_{r+1}$ is chosen sufficiently small and $|\lambda|$ sufficiently large, then $\operatorname{det}\left(\AA\left(0, \xi^{\prime}, z\right)-\right.$ $\left.\lambda I_{N}\right)$ has precisely $N_{\ell}-N_{\ell-1}$ zeros contained in $\gamma_{r, \ell}^{+}(\lambda)$. Hence since $\ell$ was chosen arbitrary, this completes the proof of the proposition.

Turning again to Propositions 3.8-3.10 and supposing henceforth that the boundary problem (1.1), (1.2) is parameter-elliptic in $\mathcal{L}$ and that $\epsilon^{0}$ has been chosen sufficiently small and $\lambda^{0}$ sufficiently large so that the conclusions of these propositions hold, let us suppose that $0 \leq r \leq d$ and that $0 \leq\left|\xi^{\prime}\right| \leq \frac{7}{8} \epsilon_{1} \mid \lambda^{\frac{1}{m_{1}}}$ if $r=0$, $\frac{1}{8} \epsilon_{r}|\lambda|^{\frac{1}{\bar{m}_{r}}} \leq\left|\xi^{\prime}\right| \leq \frac{7}{8} \epsilon_{r+1}|\lambda|^{\frac{1}{\bar{m}_{r+1}}}$ if $1 \leq r<d$, and $\frac{1}{8} \epsilon_{d}|\lambda|^{\frac{1}{m_{d}}} \leq\left|\xi^{\prime}\right|<\infty$ if $r=d$. Then it follows from $[\mathrm{V}]$ that the set of solutions of the differential equation

$$
\AA\left(0, \xi^{\prime}, D_{n}\right) u\left(x_{n}\right)-\lambda u\left(x_{n}\right)=0 \text { for } x_{n}>0
$$

which decay exponentially at $\infty$ form a vector space of dimension $N_{d}$. Furthermore, this vector space is precisely the vector space spanned by the columns of the matrix

$$
\int_{\gamma_{r}^{+}\left(\xi^{\prime}, \lambda\right)} e^{i x_{n} z}\left(\AA\left(0, \xi^{\prime}, z\right)-\lambda I_{N}\right)^{-1}\left(I_{N}, z I_{N}, \ldots, z^{m_{1}-1} I_{N}\right) d z
$$

if $r=d$, while if $r<d$, then it is precisely the direct sum of the vector space (of dimension $N_{r}$ ) spanned by the columns of (3.9) and the $N-r$ vector spaces (of dimension $N_{\ell}-N_{\ell-1}, \ell=r+1, \ldots, d$ ) spanned by the columns of each of the matrices

$$
\int_{\gamma_{r, \ell}^{+}(\lambda)} e^{i x_{n} z}\left(\AA\left(0, \xi^{\prime}, z\right)-\lambda I_{N}\right)^{-1}\left(I_{N}, z I_{N}, \ldots, z^{m_{1}-1} I_{N}\right) d z
$$


for $\ell=r+1, \ldots, d$. Note that here the contours $\gamma_{0}^{+}\left(\xi^{\prime}, \lambda\right)$ (resp. $\left.\gamma_{d}^{+}\left(\xi^{\prime}, \lambda\right)\right)$ and the $\gamma_{0, \ell}^{+}(\lambda)$ are defined in an analogous fashion to the way they were defined in the proof of Proposition 3.9 for the case $0<r<d$.

Proposition 3.11. Suppose that the boundary problem (1.1), (1.2) is parameterelliptic in $\mathcal{L}$ and that $\lambda \in \mathcal{L}$. Then we can choose the constants $\epsilon^{0}$ and $\lambda^{0}$ of Propositions 3.8-3.10 sufficiently small and sufficiently large, respectively, so that the following results hold.

If $0 \leq\left|\xi^{\prime}\right| \leq \frac{7}{8} \epsilon_{1}|\lambda|^{\frac{1}{m_{1}}}$, then

$$
\begin{aligned}
& \operatorname{rank} \int_{\gamma_{0}^{+}\left(\xi^{\prime}, \lambda\right)} \mathcal{B}^{(1,1)}\left(0, \xi^{\prime}, z\right)\left(\AA\left(0, \xi^{\prime}, z\right)-\lambda I_{N}\right)^{-1}\left(I_{N}, z I_{N}, \ldots, z^{m_{1}-1} I_{N}\right) d z \\
& \quad=N_{1}, \quad \text { and } \\
& \operatorname{rank} \int_{\gamma_{0, \ell}^{+}(\lambda)} \mathcal{B}^{(1, \ell)}\left(0, \xi^{\prime}, z\right)\left(\AA\left(0, \xi^{\prime}, z\right)-\lambda I_{N}\right)^{-1}\left(I_{N}, z I_{N}, \ldots, z^{m_{1}-1} I_{N}\right) d z \\
& \quad=N_{\ell}-N_{\ell-1} \quad \text { for } \ell=2, \ldots, d ;
\end{aligned}
$$

if $1 \leq r \leq d$ and $\frac{1}{8} \epsilon_{r}|\lambda|^{\frac{1}{m_{r}}} \leq\left|\xi^{\prime}\right| \leq \frac{7}{8} \epsilon_{r+1}|\lambda|^{\frac{1}{m_{r+1}}} \quad$ if $r<d$ or $\frac{1}{8} \epsilon_{d}|\lambda|^{\frac{1}{m_{d}}} \leq\left|\xi^{\prime}\right|<\infty$ if $r=d$, then

$$
\begin{aligned}
& \operatorname{rank} \int_{\gamma_{r}^{+}\left(\xi^{\prime}, \lambda\right)} \mathcal{B}^{(r, r)}\left(0, \xi^{\prime}, z\right)\left(\AA\left(0, \xi^{\prime}, z\right)-\lambda I_{N}\right)^{-1}\left(I_{N}, z I_{N}, \ldots, z^{m_{1}-1} I_{N}\right) d z \\
& \quad=N_{r}, \quad \text { and } \\
& \operatorname{rank} \int_{\gamma_{r, \ell}^{+}(\lambda)} \mathcal{B}^{(r, \ell)}\left(0, \xi^{\prime}, z\right)\left(\AA\left(0, \xi^{\prime}, z\right)-\lambda I_{N}\right)^{-1}\left(I_{N}, z I_{N}, \ldots, z^{m_{1}-1} I_{N}\right) d z \\
& \quad=N_{\ell}-N_{\ell-1} \quad \text { for } \ell=r+1, \ldots, d \text { if } r<d .
\end{aligned}
$$

Proof. We will only prove the proposition for the case $1 \leq r<d$; the remaining cases can be similarly treated. Accordingly, referring to the proof of Proposition 3.9 for notation, we see that for $z \in \gamma_{r}^{+}\left(\xi^{\prime}, \lambda\right)$ we have the representation

$$
\begin{array}{r}
\AA\left(0, \xi^{\prime}, z\right)-\lambda I_{N}=\left(\begin{array}{cc}
\mathcal{A}_{11}^{(r)}\left(0, \xi^{\prime}, z\right)-\lambda \widetilde{I}_{r, 0} & 0 \\
0 & -\lambda I_{N-k_{r}}
\end{array}\right) \\
+\left(\begin{array}{cc}
\lambda\left(\widetilde{I}_{r, 0}-I_{k_{r}}\right) & \mathcal{A}_{12}^{(r)}\left(0, \xi^{\prime}, z\right) \\
\mathcal{A}_{21}^{(r)}\left(0, \xi^{\prime}, z\right) & \mathcal{A}_{22}^{(r)}\left(0, \xi^{\prime}, z\right)
\end{array}\right)
\end{array}
$$

Furthermore, it follows from the proof of Proposition 3.9 that for each fixed triple $\left(\xi^{\prime}, z, \lambda\right)$, the operator $\mathcal{A}_{11}^{(r)}\left(0, \xi^{\prime}, z\right)-\lambda \widetilde{I}_{r, 0}: \mathbb{C}^{k_{r}} \rightarrow \mathbb{C}^{k_{r}}$ is bounded and invertible, while if we denote by $\widetilde{a}_{j k}\left(\xi^{\prime}, z, \lambda\right)$ the entry in the $j$-th row and $k$-th column of $\left(\mathcal{A}_{11}^{(r)}-\lambda \widetilde{I}_{r, 0}\right)^{-1}$, then $\widetilde{a}_{j k}\left(\xi^{\prime}, z, \lambda\right)$ is a rational function of its arguments and satisfies the quasi-homogeneity condition $\widetilde{a}_{j k}\left(\rho \xi^{\prime}, \rho z, \rho^{\widetilde{m}_{r}} \lambda\right)=\rho^{-t_{j}-s_{k}} \widetilde{a}_{j k}\left(\xi^{\prime}, z, \lambda\right)$ for $\rho>0$. It is now clear that $\left|\widetilde{a}_{j k}\left(\xi^{\prime}, z, \lambda\right)\right| \leq C\left\langle\xi^{\prime}, \lambda\right\rangle_{r}^{-t_{j}-s_{k}}$, where here and for the remainder of this proof $C$ denotes a generic constant that does not depend upon the variables indicated, and that the entry in the $j$-th row and $k$-th column of $\lambda\left(\widetilde{I}_{r, 0}-I_{k_{r}}\right)\left(\mathcal{A}_{11}^{(r)}\left(0, \xi^{\prime}, z\right)-\lambda \widetilde{I}_{r, 0}\right)^{-1}$ is $\lambda \widetilde{a}_{j k}\left(\xi^{\prime}, z, \lambda\right)$ if $j \leq k_{r-1}$ and is 0 otherwise. Thus if we let $\rho=\left\langle\xi^{\prime}, \lambda\right\rangle_{r}, \eta^{\prime}=\rho^{-1} \xi^{\prime}, \zeta=\rho^{-1} z, \mu=\rho^{-\widetilde{m}_{r}} \lambda$, and assume 
henceforth that $|\lambda|$ has been chosen sufficiently large, it is now a simple matter to show that if we put

$$
\begin{aligned}
& H_{11}\left(\xi^{\prime}, z, \lambda\right)=\lambda\left(\widetilde{I}_{r, 0}-I_{k_{r}}\right)\left(\mathcal{A}_{11}^{(r)}\left(0, \xi^{\prime}, z\right)-\lambda \widetilde{I}_{r, 0}\right)^{-1} \\
& =H_{11}\left(\eta^{\prime}, \zeta, \mu, \rho\right) \\
& =\mu \rho^{\widetilde{m}_{r}} \operatorname{diag}\left(\rho^{-t_{1}}, \ldots, \rho^{-t_{k_{r-1}}}, 0 \widetilde{I}_{r}\right)\left(\widetilde{a}_{j k}\left(\eta^{\prime}, \zeta, \mu\right)\right)_{j, k=1}^{k_{r}} \operatorname{diag}\left(\rho^{-s_{1}}, \ldots, \rho^{-s_{k_{r}}}\right),
\end{aligned}
$$

then the matrix $I_{k_{r}}+H_{11}\left(\xi^{\prime}, z, \lambda\right)$ is invertible and admits the representation $\left(I_{k_{r}}+H_{11}\left(\xi^{\prime}, z, \lambda\right)\right)^{-1}=I_{k_{r}}+\widetilde{H}_{11}\left(\xi^{\prime}, z, \lambda\right)$, where

$$
\begin{aligned}
\widetilde{H}_{11}\left(\xi^{\prime}, z, \lambda\right) & =\widetilde{H}_{11}\left(\eta^{\prime}, \zeta, \mu, \rho\right) \\
& =\operatorname{diag}\left(\rho^{s_{1}}, \ldots, \rho^{s_{k_{r}}}\right)\left(\widetilde{H}_{11}^{j k}\left(\eta^{\prime}, \zeta, \mu, \rho\right)\right)_{j, k=1}^{k_{r}} \operatorname{diag}\left(\rho^{-s_{1}}, \ldots, \rho^{-s_{k_{r}}}\right)
\end{aligned}
$$

and $\widetilde{H}_{11}^{j k}\left(\eta^{\prime}, \zeta, \mu, \rho\right)$ is a rational function of its arguments and $\left|\widetilde{H}_{11}^{j k}\left(\eta^{\prime}, \zeta, \mu, \rho\right)\right| \leq$ $C|\lambda|^{1-\frac{\widetilde{m}_{j}}{m_{r}}} \quad$ if $\quad j \leq k_{r-1}$ and is 0 otherwise.

Next let us put

$$
H\left(\xi^{\prime}, z, \lambda\right)=\left(\begin{array}{ll}
H_{11}\left(\xi^{\prime}, z, \lambda\right) & -H_{12}\left(\xi^{\prime}, z, \lambda\right) \\
H_{21}\left(\xi^{\prime}, z, \lambda\right) & -H_{22}\left(\xi^{\prime}, z, \lambda\right)
\end{array}\right)
$$

where $H_{12}\left(\xi^{\prime}, z, \lambda\right)=\lambda^{-1} \mathcal{A}_{12}^{(r)}\left(0, \xi^{\prime}, z\right)$,

$$
H_{21}\left(\xi^{\prime}, z, \lambda\right)=\mathcal{A}_{21}^{(r)}\left(0, \xi^{\prime}, z\right)\left(\mathcal{A}_{11}^{(r)}\left(0, \xi^{\prime}, z\right)-\lambda \widetilde{I}_{r, 0}\right)^{-1}
$$

and $H_{22}\left(\xi^{\prime}, z, \lambda\right)=\lambda^{-1} \mathcal{A}_{22}^{(r)}\left(0, \xi^{\prime}, z\right)$. Thus it follows from the foregoing results and (3.12) that

$$
\AA\left(0, \xi^{\prime}, z\right)-\lambda I_{N}=\left(I_{N}+H\left(\xi^{\prime}, z, \lambda\right)\right)\left(\begin{array}{cc}
\mathcal{A}_{11}^{(r)}\left(0, \xi^{\prime}, z\right)-\lambda \widetilde{I}_{r, 0} & 0 \\
0 & -\lambda I_{N-k_{r}},
\end{array}\right),
$$

while a factorization in the sense of Schur (see [G, Theorem 3.16, p.302]) gives

$$
\begin{aligned}
I_{N}+H\left(\xi^{\prime}, z, \lambda\right)= & \left(\begin{array}{cc}
I_{k_{r}} & 0 \\
H_{21}\left(\xi^{\prime}, z, \lambda\right)\left(I_{k_{r}}+H_{11}\left(\xi^{\prime}, z, \lambda\right)\right)^{-1} & I_{N-k_{r}}
\end{array}\right) \\
& \times\left(\begin{array}{cc}
I_{k_{r}}+H_{11}\left(\xi^{\prime}, z, \lambda\right) & 0 \\
0 & I_{N-k_{r}}-H^{\dagger}\left(\xi^{\prime}, z, \lambda\right)
\end{array}\right) \\
& \times\left(\begin{array}{ccc}
I_{k_{r}} & -\left(I_{k_{r}}+H_{11}\left(\xi^{\prime}, z, \lambda\right)\right)^{-1} H_{12}\left(\xi^{\prime}, z, \lambda\right) \\
0 & I_{N-k_{r}}
\end{array}\right),
\end{aligned}
$$

where

$$
\begin{aligned}
& H^{\dagger}\left(\xi^{\prime}, z, \lambda\right)=H_{22}\left(\xi^{\prime}, z, \lambda\right)-H_{21}\left(\xi^{\prime}, z, \lambda\right)\left(I_{k_{r}}+H_{11}\left(\xi^{\prime}, z, \lambda\right)\right)^{-1} H_{12}\left(\xi^{\prime}, z, \lambda\right) \\
& =H^{\dagger}\left(\eta^{\prime}, \zeta, \mu, \rho\right) \\
& =\operatorname{diag}\left(\rho^{s_{k_{r}+1}}, \ldots, \rho^{s_{N}}\right)\left(H_{j k}^{\dagger}\left(\eta^{\prime}, \zeta, \mu, \rho\right)\right)_{j, k=k_{r}+1}^{N} \operatorname{diag}\left(\rho^{-s_{k_{r}+1}}, \ldots, \rho^{-s_{N}}\right),
\end{aligned}
$$

and where $H_{j k}^{\dagger}\left(\eta^{\prime}, \zeta, \mu, \rho\right)$ is a rational function of its arguments and

$$
\left|H_{j k}^{\dagger}\left(\eta^{\prime}, \zeta, \mu, \rho\right)\right| \leq C\left(\epsilon_{r+1}^{\widetilde{m}_{r+1}}+|\lambda|^{\frac{\widetilde{m}_{r+1}}{\tilde{m}_{r}}-1}\right) .
$$


Thus if we henceforth suppose that $\epsilon^{0}$ has been chosen sufficiently small and $\lambda^{0}$ sufficiently large, then $I_{N-k_{r}}-H^{\dagger}\left(\xi^{\prime}, z, \lambda\right)$ is invertible and we have $\left(I_{N-k_{r}}-\right.$ $\left.H^{\dagger}\left(\xi^{\prime}, z, \lambda\right)\right)^{-1}=I_{N-k_{r}}+\widetilde{H}^{\dagger}\left(\xi^{\prime}, z, \lambda\right)$, where

$$
\begin{aligned}
& \widetilde{H}^{\dagger}\left(\xi^{\prime}, z, \lambda\right)=\widetilde{H}^{\dagger}\left(\eta^{\prime}, \zeta, \mu, \rho\right) \\
& =\operatorname{diag}\left(\rho^{s_{k_{r}+1}}, \ldots, \rho^{s_{N}}\right)\left(\widetilde{H}_{j k}^{\dagger}\left(\eta^{\prime}, \zeta, \mu, \rho\right)\right)_{j, k=k_{r}+1, \ldots, N} \operatorname{diag}\left(\rho^{-s_{k_{r}+1}}, \ldots, \rho^{-s_{N}}\right)
\end{aligned}
$$

and $\widetilde{H}_{j k}^{\dagger}\left(\eta^{\prime}, \zeta, \mu, \rho\right)$ is a rational function of its arguments and

$$
\left|\widetilde{H}_{j k}^{\dagger}\left(\eta^{\prime}, \zeta, \mu, \rho\right)\right| \leq C\left(\epsilon_{r+1}^{\widetilde{m}_{r+1}}+|\lambda|^{\frac{\widetilde{m}_{r+1}}{\tilde{m}_{r}}-1}\right) .
$$

An immediate consequence of this last result is that $\AA\left(0, \xi^{\prime}, z\right)-\lambda I_{N}$ is invertible and

$$
\begin{aligned}
& \left(\AA\left(0, \xi^{\prime}, z\right)-\lambda I_{N}\right)^{-1}=\left(\begin{array}{cc}
\left(\mathcal{A}_{11}^{(r)}\left(0, \xi^{\prime}, z\right)-\lambda \widetilde{I}_{r, 0}\right)^{-1} & 0 \\
0 & -\lambda^{-1} I_{N-k_{r}}
\end{array}\right) \\
& \times\left(I_{N}+H^{\sharp}\left(\xi^{\prime}, z, \lambda\right)\right),
\end{aligned}
$$

where

$$
\begin{aligned}
H^{\sharp}\left(\xi^{\prime}, z, \lambda\right) & =\left(\begin{array}{ll}
H_{11}^{\sharp}\left(\xi^{\prime}, z, \lambda\right) & H_{12}^{\sharp}\left(\xi^{\prime}, z, \lambda\right) \\
H_{21}^{\sharp}\left(\xi^{\prime}, z, \lambda\right) & H_{22}^{\sharp}\left(\xi^{\prime}, z, \lambda\right)
\end{array}\right), \\
H_{11}^{\sharp}\left(\xi^{\prime}, z, \lambda\right) & =\widetilde{H}_{11}\left(\xi^{\prime}, z, \lambda\right)+\left(I_{k_{r}}+\widetilde{H}_{11}\left(\xi^{\prime}, z, \lambda\right)\right) H_{12}\left(\xi^{\prime}, z, \lambda\right) H_{21}^{\sharp}\left(\xi^{\prime}, z, \lambda\right), \\
H_{12}^{\sharp}\left(\xi^{\prime}, z, \lambda\right) & =\left(I_{k_{r}}+\widetilde{H}_{11}\left(\xi^{\prime}, z, \lambda\right)\right) H_{12}\left(\xi^{\prime}, z, \lambda\right)\left(I_{N-k_{r}}+\widetilde{H}^{\dagger}\left(\xi^{\prime}, z, \lambda\right)\right), \\
H_{21}^{\sharp}\left(\xi^{\prime}, z, \lambda\right) & =-\left(I_{N-k_{r}}+\widetilde{H}^{\dagger}\left(\xi^{\prime}, z, \lambda\right)\right) H_{21}\left(\xi^{\prime}, z, \lambda\right)\left(I_{k_{r}}+\widetilde{H}_{11}\left(\xi^{\prime}, z, \lambda\right)\right), \\
H_{22}^{\sharp}\left(\xi^{\prime}, z, \lambda\right) & =\widetilde{H}^{\dagger}\left(\xi^{\prime}, z, \lambda\right) .
\end{aligned}
$$

Furthermore, we can write

$$
\begin{aligned}
& H_{11}^{\sharp}\left(\xi^{\prime}, z, \lambda\right)=H_{11}^{\sharp}\left(\eta^{\prime}, \zeta, \mu, \rho\right) \\
& =\operatorname{diag}\left(\rho^{s_{1}}, \ldots, \rho^{s_{k_{r}}}\right)\left(H_{11}^{\sharp j k}\left(\eta^{\prime}, \zeta, \mu, \rho\right)\right)_{j, k=1, \ldots, k_{r}} \operatorname{diag}\left(\rho^{-s_{1}}, \ldots, \rho^{-s_{k_{r}}}\right),
\end{aligned}
$$

where $H_{11}^{\sharp j k}\left(\eta^{\prime}, \zeta, \mu, \rho\right)$ is a rational function of its arguments and

$$
\begin{aligned}
& \left|H_{11}^{\sharp j k}\left(\eta^{\prime}, \zeta, \mu, \rho\right)\right| \leq C\left(\epsilon_{r+1}^{\widetilde{m}_{r+1}}+|\lambda|^{-1+\frac{\widetilde{m}_{r+1}}{m_{r}}}+|\lambda|^{1-\frac{\widetilde{m}_{r-1}}{m_{r}}}\right), \\
& H_{21}^{\sharp}\left(\xi^{\prime}, z, \lambda\right)=H_{21}^{\sharp}\left(\eta^{\prime}, \zeta, \mu, \rho\right) \\
& =\operatorname{diag}\left(\rho^{s_{k_{r}+1}}, \ldots, \rho^{s_{N}}\right)\left(H_{21}^{\sharp j k}\left(\eta^{\prime}, \zeta, \mu, \rho\right)\right)_{\substack{j=k_{r}+1, \ldots, N \\
k=1, \ldots, k_{r}}} \operatorname{diag}\left(\rho^{-s_{1}}, \ldots, \rho^{-s_{k_{r}}}\right),
\end{aligned}
$$

where $H_{21}^{\sharp j k}\left(\eta^{\prime}, \zeta, \mu, \rho\right)$ is a rational function of its arguments and

$$
\left|H_{21}^{\sharp j k}\left(\eta^{\prime}, \zeta, \mu, \rho\right)\right| \leq C .
$$

Let us next observe that under our assumptions concerning $\xi^{\prime}$ and $\lambda,\left(\eta^{\prime}, \mu\right) \in \Sigma_{r}$, where 


$$
\begin{aligned}
\Sigma_{r} & =\left\{\left(\widetilde{\eta}^{\prime}, \widetilde{\mu}\right) \in \mathbb{R}^{n-1} \times \mathcal{L}:\left|\widetilde{\eta}^{\prime}\right|^{2}+|\widetilde{\mu}|^{\frac{2}{m_{r}}}=1,\left|\widetilde{\eta}^{\prime}\right| \geq \frac{1}{8} \epsilon_{r}|\widetilde{\mu}|^{\frac{1}{m_{r}}}\right\} \\
& \subset \widetilde{\Sigma}_{r}=\left\{\left(\widetilde{\eta}^{\prime}, \widetilde{\mu}\right) \in \mathbb{R}^{n-1} \times \mathcal{L}:\left(1+\frac{64}{\epsilon_{r}^{2}}\right)^{-\frac{1}{2}} \leq\left|\widetilde{\eta}^{\prime}\right| \leq 1,0 \leq|\widetilde{\mu}| \leq 1\right\} .
\end{aligned}
$$

It also follows from Definition 2.6 that for $\left(\widetilde{\eta}^{\prime}, \widetilde{\mu}\right) \in \widetilde{\Sigma}_{r}, \operatorname{det}\left(\mathcal{A}_{11}^{(r)}\left(0, \widetilde{\eta}^{\prime}, \zeta\right)-\widetilde{\mu} \widetilde{I}_{r, 0}\right)$ has precisely $N_{r}$ zeros lying in $\mathbb{C}_{+}$and that there is a closed contour $\gamma_{r}^{+} \subset \mathbb{C}_{+}$, not depending upon $\widetilde{\eta}^{\prime}$ and $\widetilde{\mu}$, containing all these zeros in its interior. And it is to be understood henceforth that the contour $\gamma_{r}^{+}\left(\xi^{\prime}, \lambda\right)$ introduced above is obtained from $\gamma_{r}^{+}$by dilation by the factor $\rho$. Furthermore, as a consequence of Definition 2.6 we also know from $[\mathrm{V}]$ that if we fix our attention upon a particular pair $\left(\widetilde{\eta}^{\prime}, \widetilde{\mu}\right) \in \widetilde{\Sigma}_{r}$, then there exists matrices $J$ and $Z(\zeta)$ depending upon $\widetilde{\eta}^{\prime}$ and $\widetilde{\mu}$, such that the matrix

$$
K_{1}\left(\widetilde{\eta}^{\prime}, \widetilde{\mu}\right)=\int_{\gamma_{r}^{+}} \mathcal{B}_{r, 1}^{(r, r)}\left(0, \widetilde{\eta}^{\prime}, \zeta\right)\left(\mathcal{A}_{11}^{(r)}\left(0, \widetilde{\eta}^{\prime}, \zeta\right)-\widetilde{\mu} \widetilde{I}_{r, 0}\right)^{-1} J Z(\zeta) d \zeta
$$

has rank $N_{r}$. Here $J$ denotes a $k_{r} \times N_{r}$ matrix with the property that each of its columns has precisely one non-zero component, namely 1 , and

$$
Z(\zeta)=\operatorname{diag}\left(\zeta^{q(1)}, \ldots, \zeta^{q\left(N_{r}\right)}\right),
$$

where the $q(j)$ denote non-negative integers not exceeding $m_{1}-1$. Thus

$$
\operatorname{det} K_{1}\left(\widetilde{\eta}^{\prime}, \widetilde{\mu}\right)>c
$$

for some $c>0$.

As a consequence of (3.15), (3.16) we see that for the values of $\xi^{\prime}$ and $\lambda$ under consideration here, the rank of the matrix

$$
\int_{\gamma_{r}^{+}\left(\xi^{\prime}, \lambda\right)} \mathcal{B}_{r, 1}^{(r, r)}\left(\xi^{\prime}, z\right)\left(\mathcal{A}_{11}^{(r)}\left(0, \xi^{\prime}, z\right)-\lambda \widetilde{I}_{r, 0}\right)^{-1} J Z(z) d z
$$

is $N_{r}$ and that (3.17) can be written in the form

$$
\rho \operatorname{diag}\left(\rho^{\sigma_{1}}, \ldots, \rho^{\sigma_{N_{r}}}\right) K_{1}\left(\eta^{\prime}, \mu\right) \operatorname{diag}\left(\rho^{q(1)-s_{k(1)}}, \ldots, \rho^{q\left(N_{r}\right)-s_{k\left(N_{r}\right)}}\right),
$$

where $1 \leq k(j) \leq k_{r}$ for $1 \leq j \leq N_{r}$. Hence referring to (3.10), let us now use this fact to show that if $\epsilon^{0}$ is chosen sufficiently small and $\lambda^{0}$ sufficiently large, then the rank of

$$
\int_{\gamma_{r}^{+}\left(\xi^{\prime}, \lambda\right)} \mathcal{B}^{(r, r)}\left(0, \xi^{\prime}, z\right)\left(\AA\left(0, \xi^{\prime}, z\right)-\lambda I_{N}\right)^{-1}\left(\begin{array}{l}
J \\
0
\end{array}\right) Z(z) d z
$$

is $N_{r}$. Indeed, it follows from (3.14) that the expression (3.19) can be expressed as the sum of the expression (3.18) plus

where

$$
\begin{aligned}
& \rho \operatorname{diag}\left(\rho^{\sigma_{1}}, \ldots, \rho^{\sigma_{N_{r}}}\right)\left(\int_{\gamma_{r}^{+}} \widetilde{K}_{2}\left(\eta^{\prime}, \zeta, \mu, \rho\right) J Z(\zeta) d \zeta\right) \\
& \times \operatorname{diag}\left(\rho^{q(1)-s_{k(1)}}, \ldots, \rho^{q\left(N_{r}\right)-s_{k\left(N_{r}\right)}}\right),
\end{aligned}
$$

$$
\begin{aligned}
\widetilde{K}_{2}\left(\eta^{\prime}, \zeta, \mu, \rho\right) & =\mathcal{B}_{11}^{(r, r)}\left(0, \eta^{\prime}, \zeta\right)\left(\mathcal{A}_{11}^{(r)}\left(0, \eta^{\prime}, \zeta\right)-\mu \widetilde{I}_{r, 0}\right)^{-1} H_{11}^{\sharp}\left(\eta^{\prime}, \zeta, \mu, \rho\right) \\
& -\left(\mu \rho^{\widetilde{m}_{r}}\right)^{-1} \mathcal{B}_{11}^{(r, r)}\left(0, \eta^{\prime}, \zeta\right) \operatorname{diag}\left(\rho^{m_{k_{r}+1}}, \ldots, \rho^{m_{N}}\right) H_{21}^{\sharp}\left(\eta^{\prime}, \zeta, \mu, \rho\right)
\end{aligned}
$$


is an $N_{r} \times N_{r}$ matrix function whose entry in the $j$-th row and $k$-th column, $k_{j k}\left(\eta^{\prime}, \zeta, \mu, \rho\right)$ is a rational function of its arguments and

$$
\left|k_{j k}\left(\eta^{\prime}, \zeta, \mu, \rho\right)\right| \leq C\left(\epsilon_{r+1}^{\widetilde{m}_{r+1}}+|\lambda|^{\frac{\widetilde{m}_{r_{r+1}}}{m_{r}}-1}+|\lambda|^{1-\frac{\widetilde{m}_{r-1}}{\tilde{m}_{r}}}\right) .
$$

It follows immediately from these results that if we suppose that $\epsilon^{0}$ is chosen sufficiently small and $\lambda^{0}$ sufficiently large, then the matrix (3.19) has rank $N_{r}$, which proves the proposition for the case under consideration.

Suppose next that $r<\ell \leq d$ and that $z \in \gamma_{r, \ell}^{+}(\lambda)$. Then as a consequence of our hypotheses we know that $\left(\mathcal{A}_{11}^{(\ell)}(0,0, z)-\lambda \widetilde{I}_{\ell, 0}\right)$ is invertible and if we let $\left(\mathcal{A}_{11}^{(\ell)}(0,0, z)-\lambda \widetilde{I}_{\ell, 0}\right)^{-1}=\left(\widetilde{a}_{j k}^{(\ell)}(z, \lambda)\right)_{j, k=1}^{k_{\ell}}$, then $\widetilde{a}_{j k}^{(\ell)}(z, \lambda)$ is a rational functional function of its arguments and satisfies the quasi-homogeneity condition $\widetilde{a}_{j k}^{(\ell)}\left(\rho z, \rho^{\tilde{m}_{\ell}} \lambda\right)=\rho^{-t_{j}-s_{k}} \widetilde{a}_{j k}^{(\ell)}(z, \lambda)$ for $\rho>0$. Let us now introduce the matrix function $H^{(\ell)}\left(\xi^{\prime}, z, \lambda\right)=\left(H_{j k}^{(\ell)}\left(\xi^{\prime}, z, \lambda\right)\right)_{j, k=1}^{2}$, where

$$
\begin{aligned}
H_{11}^{(\ell)}\left(\xi^{\prime}, z, \lambda\right)= & \left(\mathcal{A}_{11}^{(\ell)}\left(0, \xi^{\prime}, z\right)-\mathcal{A}_{11}^{(\ell)}(0,0, z)+\lambda\left(\widetilde{I}_{\ell, 0}-I_{k_{\ell}}\right)\right. \\
& \times\left(\mathcal{A}_{11}^{(\ell)}(0,0, z)-\lambda \widetilde{I}_{\ell, 0}\right)^{-1},
\end{aligned}
$$

and if $\ell<d$, then $H_{12}^{\ell}\left(\xi^{\prime}, z, \lambda\right)=\lambda^{-1} \mathcal{A}_{12}^{(\ell)}\left(\xi^{\prime}, z\right)$,

$$
H_{21}^{(\ell)}\left(\left(\xi^{\prime}, z, \lambda\right)=\mathcal{A}_{21}^{(\ell)}\left(\xi^{\prime}, z\right)\left(\mathcal{A}_{11}^{(\ell)}(0,0, z)-\lambda \widetilde{I}_{\ell, 0}\right)^{-1},\right.
$$

and $H_{22}^{(\ell)}\left(\xi^{\prime}, z, \lambda\right)=\lambda^{-1} \mathcal{A}_{22}^{(\ell)}\left(\xi^{\prime}, z\right)$. Then proceeding as in the previous case, we can write

$$
\begin{aligned}
& H_{11}^{(\ell)}\left(\xi^{\prime}, z, \lambda\right)=H_{11}^{(\ell)}\left(\eta_{\ell}^{\prime}, \zeta_{\ell}, \mu_{\ell}, \rho_{\ell}\right) \\
& =\operatorname{diag}\left(\rho_{\ell}^{s_{1}}, \ldots, \rho_{\ell}^{s_{k_{\ell}}}\right)\left(H_{11}^{(\ell j k)}\left(\eta_{\ell}^{\prime}, \zeta_{\ell}, \mu_{\ell}, \rho_{\ell}\right)\right)_{j, k=1}^{k_{\ell}} \operatorname{diag}\left(\rho_{\ell}^{-s_{1}}, \ldots, \rho_{\ell}^{-s_{k_{\ell}}}\right),
\end{aligned}
$$

where $\rho_{\ell}=|\lambda|^{\frac{1}{m_{\ell}}}, \eta_{\ell}^{\prime}=\rho_{\ell}^{-1} \xi^{\prime}, \zeta_{\ell}=\rho_{\ell}^{-1} z, \mu_{\ell}=\rho_{\ell}^{-\widetilde{m}_{\ell}} \lambda$, and $H_{11}^{(\ell) j k}\left(\eta_{\ell}^{\prime}, \zeta_{\ell}, \mu_{\ell}, \rho_{\ell}\right)$ is a rational functional of its arguments and

$$
\left|H_{11}^{(\ell) j k}\left(\eta_{\ell}^{\prime}, \zeta_{\ell}, \mu_{\ell}, \rho_{\ell}\right)\right| \leq C\left(\delta_{r+1, \ell} \epsilon_{r+1}+\rho_{\ell}^{\tilde{m}_{\ell}-\widetilde{m}_{\ell-1}}\right),
$$

where here and below $C$ denotes a generic constant which does not depend upon any of the variables indicated. Hence it follows that for $\epsilon^{0}$ sufficiently small and $\lambda^{0}$ sufficiently large, $I_{k_{\ell}}+H_{11}^{(\ell)}\left(\xi^{\prime}, z, \lambda\right)$ is invertible and we have

$$
\begin{aligned}
\left(I_{k_{\ell}}+H_{11}^{(\ell)}\left(\xi^{\prime}, z, \lambda\right)\right)^{-1} & =\operatorname{diag}\left(\rho_{\ell}^{s_{1}}, \ldots, \rho_{\ell}^{s_{\ell}}\right)\left(I_{k_{\ell}}+\widetilde{H}^{(\ell)}\left(\eta_{\ell}^{\prime}, \zeta_{\ell}, \mu_{\ell}, \rho_{\ell}\right)\right) \\
& \times \operatorname{diag}\left(\rho_{\ell}^{-s_{1}}, \ldots, \rho_{\ell}^{-s_{k_{\ell}}}\right)
\end{aligned}
$$

where $\widetilde{H}_{11}^{(\ell)}\left(\eta_{\ell}^{\prime}, \zeta_{\ell}, \mu_{\ell}, \rho_{\ell}\right)=\left(\widetilde{H}_{11}^{(\ell) j k}\left(\eta_{\ell}^{\prime}, \zeta_{\ell}, \mu_{\ell}, \rho_{\ell}\right)\right)_{j, k=1}^{k_{\ell}}$ and $\widetilde{H}_{11}^{(\ell) j k}\left(\eta_{\ell}^{\prime}, \zeta_{\ell}, \mu_{\ell}, \rho_{\ell}\right)$ is a rational functional function of its arguments and is bounded in modulus by the expression on the right side of (3.21). 
Suppose next that $\ell<d$. Then proceeding as we did in our previous case, let us now put

$H^{\dagger(\ell)}\left(\xi^{\prime}, z, \lambda\right)=H_{22}^{(\ell)}\left(\xi^{\prime}, z, \lambda\right)-H_{21}^{(\ell)}\left(\xi^{\prime}, z, \lambda\right)\left(I_{k_{\ell}}+H_{11}^{(\ell)}\left(\xi^{\prime}, z, \lambda\right)\right)^{-1} H_{12}^{(\ell)}\left(\xi^{\prime}, z, \lambda\right)$.

Then we can write

$$
\begin{aligned}
& H^{\dagger(\ell)}\left(\xi^{\prime}, z, \lambda\right)=H^{\dagger(\ell)}\left(\eta_{\ell}^{\prime}, \zeta_{\ell}, \mu_{\ell}, \rho_{\ell}\right) \\
& =\operatorname{diag}\left(\rho_{\ell}^{s_{k_{\ell}+1}}, \ldots, \rho_{\ell}^{s_{N}}\right)\left(H_{j k}^{\dagger(\ell)}\left(\eta_{\ell}^{\prime}, \zeta_{\ell}, \mu_{\ell}, \rho_{\ell}\right)\right)_{j, k=k_{\ell}+1}^{N} \operatorname{diag}\left(\rho_{\ell}^{-s_{k_{\ell}+1}}, \ldots, \rho_{\ell}^{-s_{N}}\right),
\end{aligned}
$$

where it follows from its definition that $H_{j k}^{\dagger(\ell)}\left(\eta_{\ell}^{\prime}, \zeta_{\ell}, \mu_{\ell}, \rho_{\ell}\right)$ is a rational function of its arguments and is bounded in modulus by $C|\lambda|^{-1+\frac{\widetilde{m}_{\ell+1}}{\bar{m}_{\ell}}}$. Hence if we suppose that $\lambda^{0}$ is sufficiently large, then $I_{N-k_{\ell}}-H^{\dagger(\ell)}\left(\xi^{\prime}, z, \lambda\right)$ is invertible, and if we write

$$
\left(I_{N-k_{\ell}}-H^{\dagger(\ell)}\left(\xi^{\prime}, z, \lambda\right)\right)^{-1}=I_{N-k_{\ell}}+\widetilde{H}^{\dagger(\ell)}\left(\xi^{\prime}, z, \lambda\right)
$$

and

$$
\begin{aligned}
& \widetilde{H}^{\dagger(\ell)}\left(\xi^{\prime}, z, \lambda\right)=\widetilde{H}^{\dagger(\ell)}\left(\eta_{\ell}^{\prime}, \zeta_{\ell}, \mu_{\ell}, \rho_{\ell}\right) \\
& =\operatorname{diag}\left(\rho_{\ell}^{s_{k_{\ell}+1}}, \ldots, \rho_{\ell}^{s_{N}}\right)\left(\widetilde{H}_{j k}^{\dagger(\ell)}\left(\eta_{\ell}^{\prime}, \zeta_{\ell}, \mu_{\ell}, \rho_{\ell}\right)\right)_{j, k=k_{\ell}+1}^{N} \operatorname{diag}\left(\rho_{\ell}^{-s_{k_{\ell}+1}}, \ldots, \rho_{\ell}^{-s_{N}}\right),
\end{aligned}
$$

then $\widetilde{H}_{j k}^{\dagger(\ell)}\left(\eta_{\ell}^{\prime}, \zeta_{\ell}, \mu_{\ell}, \rho_{\ell}\right)$ is a rational function of its arguments and is bounded in modulus by $C|\lambda|^{-1+\frac{\widetilde{m}_{\ell+1}}{\bar{m}_{\ell}}} \rho_{\ell}^{s_{j}-s_{k}}$.

In light of these definitions, we can now proceed as in the first part of the proof to show that for $\ell<d$,

$$
\begin{aligned}
\left(\AA\left(0, \xi^{\prime}, z\right)-\lambda I_{N}\right)^{-1} & =\left(\begin{array}{cc}
\left(\mathcal{A}_{11}^{(\ell)}(0,0, z)-\lambda \widetilde{I}_{\ell, 0}\right)^{-1} & 0 \\
0 & -\lambda^{-1} I_{N-k_{\ell}}
\end{array}\right) \\
& \times\left(I_{N}+H^{\sharp(\ell)}\left(\xi^{\prime}, z, \lambda\right)\right),
\end{aligned}
$$

where

$$
\begin{aligned}
& H^{\sharp(\ell)}\left(\xi^{\prime}, z, \lambda\right)=\left(H_{j k}^{\sharp(\ell)}\left(\xi^{\prime}, z, \lambda\right)\right)_{j, k=1}^{2}, \\
& H_{11}^{\sharp(\ell)}\left(\xi^{\prime}, z, \lambda\right)=\widetilde{H}_{11}^{(\ell)}\left(\xi^{\prime}, z, \lambda\right)+\left(I_{k_{\ell}}+\widetilde{H}_{11}^{(\ell)}\left(\xi^{\prime}, z, \lambda\right)\right) H_{12}^{(\ell)}\left(\xi^{\prime}, z, \lambda\right) H_{21}^{\sharp(\ell)}\left(\xi^{\prime}, z, \lambda\right), \\
& H_{12}^{\sharp(\ell)}\left(\xi^{\prime}, z, \lambda\right)=\left(I_{k_{\ell}}+\widetilde{H}_{11}^{(\ell)}\left(\xi^{\prime}, z, \lambda\right)\right) H_{12}^{(\ell)}\left(\xi^{\prime}, z, \lambda\right)\left(I_{N-k_{\ell}}+\widetilde{H}^{\dagger(\ell)}\left(\xi^{\prime}, z, \lambda\right)\right), \\
& H_{21}^{\sharp(\ell)}\left(\xi^{\prime}, z, \lambda\right)=-\left(I_{N-k_{\ell}}+\widetilde{H}^{\dagger(\ell)}\left(\xi^{\prime}, z, \lambda\right)\right) H_{21}^{(\ell)}\left(\xi^{\prime}, z, \lambda\right)\left(I_{k_{\ell}}+\widetilde{H}_{11}^{(\ell)}\left(\xi^{\prime}, z, \lambda\right)\right), \\
& H_{22}^{\sharp(\ell)}\left(\xi^{\prime}, z, \lambda\right)=I_{N-k_{\ell}}+\widetilde{H}^{\dagger(\ell)}\left(\xi^{\prime}, z, \lambda\right),
\end{aligned}
$$

and where these results are to be modified in an obvious way if $\ell=d$. Note also that

$$
\begin{aligned}
& H_{11}^{\sharp(\ell)}\left(\xi^{\prime}, z, \lambda\right)=H_{11}^{\sharp(\ell)}\left(\eta_{\ell}^{\prime}, \zeta_{\ell}, \mu_{\ell}, \rho_{\ell}\right) \\
& =\operatorname{diag}\left(\rho_{\ell}^{s_{1}}, \ldots, \rho_{\ell}^{s_{k_{\ell}}}\right)\left(H_{11, j k}^{\sharp(\ell)}\left(\eta_{\ell}^{\prime}, \zeta_{\ell}, \mu_{\ell}, \rho_{\ell}\right)\right)_{j, k=1}^{k_{\ell}} \operatorname{diag}\left(\rho_{\ell}^{-s_{1}}, \ldots, \rho_{\ell}^{-s_{k_{\ell}}}\right),
\end{aligned}
$$


where $H_{11, j k}^{\sharp(\ell)}\left(\eta_{\ell}^{\prime}, \zeta_{\ell}, \mu_{\ell}, \rho_{\ell}\right)$ is a rational functional function of its arguments and is bounded in modulus by $C\left(|\lambda|^{-1+\frac{\widetilde{m}_{\ell+1}}{\bar{m}_{\ell}}}+|\lambda|^{1-\frac{\widetilde{m}_{\ell-1}}{\bar{m}_{\ell}}}+\epsilon_{r+1}\right)$, and if $\ell<d$, then

$$
\begin{aligned}
H_{21}^{\sharp(\ell)}\left(\xi^{\prime}, z, \lambda\right) & =H_{21}^{\sharp(\ell)}\left(\eta^{\prime}, \zeta_{\ell}, \mu_{\ell}, \rho_{\ell}\right) \\
& =\operatorname{diag}\left(\rho_{\ell}^{s_{k_{\ell}+1}}, \ldots, \rho_{\ell}^{s_{N}}\right)\left(H_{21, j k}^{\sharp(\ell)}\left(\eta_{\ell}^{\prime}, \zeta_{\ell}, \mu_{\ell}, \rho_{\ell}\right)\right)_{\substack{j=k_{\ell}+1, \ldots, N \\
k=1, \ldots, k_{\ell}}} \\
& \times \operatorname{diag}\left(\rho_{\ell}^{-s_{1}}, \ldots, \rho_{\ell}^{-s_{k_{\ell}}}\right),
\end{aligned}
$$

where $H_{21, j k}^{\sharp(\ell)}\left(\eta_{\ell}^{\prime}, \zeta_{\ell}, \mu_{\ell}, \rho_{\ell}\right)$ is a rational function of its arguments and is bounded in modulus by $C$.

Let us next observe that under our assumptions concerning $\xi^{\prime}$ and $\lambda, \mu_{\ell} \in$ $\mathcal{L}_{1}=\{\widetilde{\mu} \in \mathcal{L}|| \widetilde{\mu} \mid=1\}$. It also follows from Definition 2.4 that for $\widetilde{\mu} \in \mathcal{L}_{1}$, $\operatorname{det}\left(\mathcal{A}_{11}^{(\ell)}(0,0, \zeta)-\widetilde{\mu} \widetilde{I}_{\ell, 0}\right)$ has precisely $N_{\ell}-N_{\ell-1}$ zeros lying in $\mathbb{C}_{+}$and that there is a closed contour $\gamma_{r, \ell}^{+} \subset \mathbb{C}_{+}$, not depending upon $\widetilde{\mu}$, containing all these zeros in its interior. And it is to be understood henceforth that the contour $\gamma_{r, \ell}^{+}(\lambda)$ introduced above is obtained from $\gamma_{r, \ell}^{+}$by dilation by the factor $\rho_{\ell}$. Furthermore, as consequence of Definition 2.6 it also follows from [V] that if we fix our attention upon a particular $\tilde{\mu} \in \mathcal{L}_{1}$, then there exist matrices $J^{(\ell)}$ and $Z^{(\ell)}\left(\zeta_{\ell}\right)$, depending upon $\widetilde{\mu}$, such that

$$
K_{1}^{(\ell)}(0, \widetilde{\mu})=\int_{\gamma_{r, \ell}^{+}} \mathcal{B}_{\ell, 1}^{(r, \ell)}\left(0,0, \zeta_{\ell}\right)\left(\mathcal{A}_{11}^{(\ell)}\left(0,0, \zeta_{\ell}\right)-\widetilde{\mu} \widetilde{I}_{\ell, 0}\right)^{-1} J^{(\ell)} Z^{(\ell)}\left(\zeta_{\ell}\right) d \zeta_{\ell}
$$

has rank $N_{\ell}-N_{\ell-1}$. Here $J^{(\ell)}$ is a $k_{\ell} \times\left(N_{\ell}-N_{\ell-1}\right)$ matrix having the same properties as the matrix $J$ of $(3.15)$ and $Z^{(\ell)}\left(\zeta_{\ell}\right)=\operatorname{diag}\left(\zeta_{\ell}^{q(1)}, \ldots, \zeta_{\ell}^{q\left(N_{\ell}-N_{\ell-1}\right)}\right)$ and the $q(j)$ are defined as in the text following (3.15). Then

$$
\operatorname{det} K_{1}^{(\ell)}(0, \widetilde{\mu})>c^{(\ell)}
$$

for some constant $c^{(\ell)}>0$.

As a consequence of (3.23) and (3.24) we see that for the values of $\xi^{\prime}$ and $\lambda$ under consideration here the rank of the matrix

$$
\int_{\gamma_{r, \ell}^{+}(\lambda)} \mathcal{B}_{\ell, 1}^{(r, \ell)}(0, z)\left(\mathcal{A}_{11}^{(\ell)}(0,0, z)-\lambda \widetilde{I}_{\ell, 0}\right)^{-1} J^{(\ell)} Z^{(\ell)}(z) d z
$$

is $N_{\ell}-N_{\ell-1}$ and that (3.25) can be written in the form

$$
\begin{aligned}
& \rho_{\ell} \operatorname{diag}\left(\rho_{\ell}^{\sigma_{N_{\ell-1}+1}}, \ldots, \rho_{\ell}^{\sigma_{N_{\ell}-N_{\ell-1}}}\right) K_{1}^{(\ell)}\left(0, \mu_{\ell}\right) \\
& \quad \times \operatorname{diag}\left(\rho_{\ell}^{q(1)-s_{k(1)}}, \ldots, \rho_{\ell}^{q\left(N_{\ell}-N_{\ell-1}\right)-s_{k\left(N_{\ell}-N_{\ell-1}\right)}}\right),
\end{aligned}
$$

where $1 \leq k(j) \leq k_{\ell}$ for $1 \leq j \leq N_{\ell}-N_{\ell-1}$. Hence referring to (3.11), let us now use these facts to show that if $\epsilon^{0}$ is sufficiently small and $\lambda^{0}$ is sufficiently large, then the rank of the matrix

$$
\int_{\gamma_{r, \ell}^{+}(\lambda)} \mathcal{B}^{(r, \ell)}\left(0, \xi^{\prime}, z\right)\left(\AA\left(0, \xi^{\prime}, z\right)-\lambda I_{N}\right)^{-1}\left(\begin{array}{c}
J^{(\ell)} \\
0
\end{array}\right) Z^{(\ell)}(z) d z
$$


is $N_{\ell}-N_{\ell-1}$. Indeed it follows from (3.22) that the expression (3.27) can be expressed as the sum of the expression (3.26) plus the expression

$$
\begin{gathered}
\rho_{\ell} \operatorname{diag}\left(\rho_{\ell}^{\sigma_{N_{\ell-1}+1}}, \ldots, \rho_{\ell}^{\sigma_{N_{\ell}}}\right)\left(\int_{\gamma_{r, \ell}^{+}} \widetilde{K}_{2}^{(\ell)}\left(\eta_{\ell}^{\prime}, \zeta_{\ell}, \mu_{\ell}, \rho_{\ell}\right) J^{(\ell)} Z^{(\ell)}\left(\zeta_{\ell}\right) d \zeta_{\ell}\right) \\
\times \operatorname{diag}\left(\rho_{\ell}^{q(1)-s_{k(1)}}, \ldots, \rho_{\ell}^{q\left(N_{\ell}-N_{\ell-1}\right)-s_{k\left(N_{\ell}-N_{\ell-1}\right)}}\right)
\end{gathered}
$$

where

$$
\begin{aligned}
\widetilde{K}_{2}^{(\ell)}\left(\eta_{\ell}^{\prime}, \zeta_{\ell}, \mu_{\ell}, \rho_{\ell}\right) & \left.=\left(\mathcal{B}_{\ell, 1}^{(r, \ell)}\left(0, \eta_{\ell}^{\prime}, \zeta_{\ell}\right)-\mathcal{B}_{11}^{(r, \ell)}\right)\left(0,0, \zeta_{\ell}\right)\right)\left(\mathcal{A}_{11}^{(\ell)}\left(0,0, \zeta_{\ell}\right)-\mu_{\ell} \widetilde{I}_{\ell, 0}\right)^{-1} \\
& +\mathcal{B}_{\ell, 1}^{(r, \ell)}\left(0, \eta_{\ell}^{\prime}, \zeta_{\ell}\right)\left(\mathcal{A}_{11}^{(\ell)}\left(0,0, \zeta_{\ell}\right)-\mu_{\ell} \widetilde{I}_{\ell, 0}\right)^{-1} H_{11}^{\sharp(\ell)}\left(\eta_{\ell}^{\prime}, \zeta_{\ell}, \mu_{\ell}, \rho_{\ell}\right) \\
& -\left(1-\delta_{\ell, d}\right)\left(\mu_{\ell} \rho_{\ell}^{\tilde{m}_{\ell}}\right)^{-1} \mathcal{B}_{\ell, 2}^{(r, \ell)}\left(0, \eta_{\ell}^{\prime}, \zeta_{\ell}\right) H_{21}^{\sharp(\ell)}\left(\eta_{\ell}^{\prime}, \zeta_{\ell}, \mu_{\ell}, \rho_{\ell}\right)
\end{aligned}
$$

is an $\left(N_{\ell}-N_{\ell-1}\right) \times\left(N_{\ell}-N_{\ell-1}\right)$ matrix function whose entry in the $j$-th row and $k$-th column, $k_{j k}^{(\ell)}\left(\eta_{\ell}^{\prime}, \zeta_{\ell}, \mu_{\ell}, \rho_{\ell}\right)$, is a rational function of its arguments and

$$
\begin{aligned}
& \left|k_{j k}^{(\ell)}\left(\eta_{\ell}^{\prime}, \zeta_{\ell}, \mu_{\ell}, \rho_{\ell}\right)\right| \leq \\
& \quad C\left(\delta_{r+1, \ell} \epsilon_{r+1}+|\lambda|^{\frac{1}{m_{\ell-1}}-\frac{1}{m_{\ell}}}+|\lambda|^{1-\frac{\widetilde{m}_{\ell-1}}{m_{\ell}}}+\left(1-\delta_{\ell, d}\right)|\lambda|^{\frac{\widetilde{m}_{\ell+1}}{m_{\ell}}-1}\right) .
\end{aligned}
$$

It follows immediately from these results that if we suppose that $\epsilon^{0}$ and $\lambda^{0}$ are sufficiently large and small, respectively, then the matrix (3.27) has rank $N_{\ell}-N_{\ell-1}$, and this completes the proof of the proposition.

In light of Proposition 3.11 and from what was said in the text preceding that proposition, we are now in a position to present some results pertaining to the solutions of (3.8). To this end let us remark for later use that the contour $\gamma_{r}^{+}$and the set $\Sigma_{r}$ for $r=0$ and $r=d$ are defined in an analogous manner to the way they were in the proof of Proposition 3.11, except that we now take $\Sigma_{0}=\left\{\left(\xi^{\prime}, \lambda\right) \in\right.$ $\left.\mathbb{R}^{n-1} \times \mathcal{L}:\left|\xi^{\prime}\right|^{2}+|\lambda|^{\frac{2}{m_{1}}}=1\right\}$. A similar remark holds for $\gamma_{0, \ell}^{+}$.

Proposition 3.12. Suppose that the hypotheses of Proposition 3.11 hold and that $\epsilon^{0}$ and $\lambda^{0}$ have been chosen small enough and large enough, respectively, so that the conclusions of that proposition hold. Suppose also that either

(i) $r=0$ and $\left|\xi^{\prime}\right| \leq \frac{7}{8} \epsilon_{1}|\lambda|^{1 / \widetilde{m}_{1}}$, or

(ii) $1 \leq r<d$ and $\frac{1}{8} \epsilon_{r}|\lambda|^{1 / \widetilde{m}_{r}} \leq\left|\xi^{\prime}\right| \leq \frac{7}{8} \epsilon_{r+1}|\lambda|^{1 / \widetilde{m}_{r+1}}$, or

(iii) $r=d$ and $\frac{1}{8} \epsilon_{d}|\lambda|^{1 / \widetilde{m}_{d}} \leq\left|\xi^{\prime}\right|<\infty$.

Then the differential equation (3.8) has $\tilde{N}$ linearly independent solutions, $\left\{w^{(r, \nu)}\left(\xi^{\prime}, x_{n}, \lambda\right)\right\}_{\nu=1}^{\widetilde{N}}$, which decay exponentially at $\infty$ and satisfy

$$
\mathcal{B}^{(1, \ell)}\left(0, \xi^{\prime}, D_{n}\right) W^{(0, \ell)}\left(\xi^{\prime}, 0, \lambda\right)=I_{N_{\ell}-N_{\ell-1}} \quad \text { for } \ell=1, \ldots, d,
$$

if $r=0$,

$$
\mathcal{B}^{(r, \ell)}\left(0, \xi^{\prime}, D_{n}\right) W^{(r, \ell)}\left(\xi^{\prime}, 0, \lambda\right)=I_{N_{\ell}-N_{r, \ell-1}^{\dagger}} \quad \text { for } \ell=r, \ldots, d,
$$

if $1 \leq r<d$,

$$
\mathcal{B}^{(d, d)}\left(0, \xi^{\prime}, D_{n}\right) W^{(d, d)}\left(\xi^{\prime}, 0, \lambda\right)=I_{N_{d}}
$$


R. DENK AND M. FAIERMAN

where $N_{r, \ell-1}^{\dagger}=\left(1-\delta_{\max \{r, 1\}, \ell}\right) N_{\ell-1}, W^{(0, \ell)}\left(\xi^{\prime}, x_{n}, \lambda\right)$ denotes the $N \times\left(N_{\ell}-N_{\ell-1}\right)$ matrix function whose columns are precisely the $w^{(0, \nu)}\left(\xi^{\prime}, x_{n}, \lambda\right)$ for $\nu=N_{\ell-1}+$ $1, \ldots, N_{\ell}$, while for $r>0, W^{(r, \ell)}\left(\xi^{\prime}, x_{n}, \lambda\right)$ denotes the $N \times\left(N_{\ell}-N_{r, \ell-1}^{\dagger}\right)$ matrix function whose columns are precisely the $w^{(r, \nu)}\left(\xi^{\prime}, x_{n}, \lambda\right)$ for $\nu=N_{r, \ell-1}^{\dagger}+1, \ldots, N_{\ell}$. Furthermore, we have the representations (here we let $r^{\dagger}=\max \{r, 1\}$ )

$$
\begin{gathered}
W^{\left(r, r^{\dagger}\right)}\left(\xi^{\prime}, x_{n}, \lambda\right)=\int_{\gamma_{r}^{+}\left(\xi^{\prime}, \lambda\right)} e^{i x_{n} z}\left(\AA\left(0, \xi^{\prime}, z\right)-\lambda I_{N}\right)^{-1} \bar{G}^{\left(r, r^{\dagger}\right)}\left(\xi^{\prime}, z, \lambda\right) d z, \\
W^{(r, \ell)}\left(\xi^{\prime}, x_{n}, \lambda\right)=\int_{\gamma_{r, \ell}^{+}(\lambda)} e^{i x_{n} z}\left(\stackrel{\AA}{A}\left(0, \xi^{\prime}, z\right)-\lambda I_{N}\right)^{-1} \bar{G}^{(r, \ell)}\left(\xi^{\prime}, z, \lambda\right) d z, \\
\text { for } \ell=r^{\dagger}+1, \ldots, d \text { if } r<d,
\end{gathered}
$$

where for $r^{\dagger} \leq \ell \leq d$

$$
\begin{aligned}
& \bar{G}^{(r, \ell)}\left(\xi^{\prime}, z, \lambda\right)=\left(\begin{array}{c}
\widetilde{G}^{(r, \ell)}\left(\eta_{r, \ell}^{\prime}, \zeta_{r, \ell}, \mu_{r, \ell}, \rho_{r, \ell}\right) \\
0 I_{N-k_{\ell}}
\end{array}\right), \\
& \widetilde{G}^{(r, \ell)}\left(\eta_{r, \ell}^{\prime}, \zeta_{r, \ell}, \mu_{r, \ell}, \rho_{r, \ell}\right)=\rho_{r, \ell}^{-1} \operatorname{diag}\left(\rho_{r, \ell}^{s_{1}}, \ldots, \rho_{r, \ell}^{s_{k_{\ell}}}\right) K^{(r, \ell)}\left(\eta_{r, \ell}^{\prime}, \zeta_{r, \ell}, \mu_{r, \ell}, \rho_{r, \ell}\right) \\
& \times \operatorname{diag}\left(\rho_{r, \ell}^{-\sigma_{N_{r, \ell-1}^{\dagger}+1}}, \ldots, \rho_{r, \ell}^{-\sigma_{N_{\ell}}}\right), \\
& K^{(r, \ell)}\left(\eta_{r, \ell}^{\prime}, \zeta_{r, \ell}, \mu_{r, \ell}, \rho_{r, \ell}\right)=\left(K_{j k}^{(r, \ell)}\left(\eta_{r, \ell}^{\prime}, \zeta_{r, \ell}, \mu_{r, \ell}, \rho_{r, \ell}\right)\right)_{\substack{j=1, \ldots, k_{\ell} \\
k=N_{r, \ell-1}^{\dagger}+1, \ldots, N_{\ell}}}^{j},
\end{aligned}
$$
and

such that for each pair $j, k, K_{j k}^{(r, \ell)}\left(\eta_{r, \ell}^{\prime}, \zeta_{r, \ell}, \mu_{r, \ell}, \rho_{r, \ell}\right)$ is a finite sum of a product of a power of $\zeta_{r, \ell}$ and an expression of rational type in $\eta_{r, \ell}^{\prime}, \zeta_{r, \ell}, \mu_{r, \ell}$ and $\rho_{r, \ell}$ (i.e., a rational function of terms which are integrals over $\delta_{r^{\dagger}, \ell} \gamma_{r}^{+}+\left(1-\delta_{r^{\dagger}, \ell}\right) \gamma_{r, \ell}^{+}$of rational functions of the components of $\eta_{r, \ell}^{\prime}, \zeta_{r, \ell}, \mu_{r, \ell}$ and $\left.\rho_{r, \ell}\right)$ and is bounded in modulus by a constant depending only upon $\eta_{r, r^{\dagger}}^{\prime}$ and $\mu_{r, r^{\dagger}}\left(\in \Sigma_{r}\right)$ if $\ell=r^{\dagger}$ and only upon $\rho_{r, \ell}\left(\in \mathcal{L}_{1}\right)$ if $\ell>r^{\dagger}$, and lastly the block $0 I_{N-k_{\ell}}$ is to be omitted when $\ell=d$.

Proof. We will only prove the proposition for the case $1 \leq r<d$ as the proofs of the other cases are similar. Accordingly, returning to the proof of Proposition 3.11 and employing the notation of that proposition, let $\Lambda\left(\xi^{\prime}, \lambda\right)$ denote the expression (3.19). Then we know from the proof of Proposition 3.11 that

$$
\begin{aligned}
\Lambda\left(\xi^{\prime}, \lambda\right) & =\rho \operatorname{diag}\left(\rho^{\sigma_{1}}, \ldots, \rho^{\sigma_{N_{r}}}\right)\left(K_{1}\left(\eta^{\prime}, \mu\right)+K_{2}\left(\eta^{\prime}, \mu, \rho\right)\right) \\
& \times \operatorname{diag}\left(\rho^{q(1)-s_{k(1)}}, \ldots, \rho^{q\left(N_{r}\right)-s_{k\left(N_{r}\right)}}\right),
\end{aligned}
$$

where $K_{2}\left(\eta^{\prime}, \mu, \rho\right)=\int_{\gamma_{r}^{+}} \widetilde{K}_{2}\left(\eta^{\prime}, \zeta, \mu, \rho\right) J Z(\zeta) d \zeta$ and where we refer to the text following (3.15) for notation. Hence bearing in mind (3.16) and (3.20), it is an immediate consequence of Proposition 3.11 that $\Lambda\left(\xi^{\prime}, \lambda\right)$ is invertible and

$$
\begin{aligned}
\Lambda\left(\xi^{\prime}, \lambda\right)^{-1}= & \rho^{-1} \operatorname{diag}\left(\rho^{s_{k(1)}-q(1)}, \ldots, \rho^{s_{k\left(N_{r}\right)}-q_{N(r)}}\right) \\
& \times\left(I_{N_{r}}+K_{3}\left(\eta^{\prime}, \mu, \rho\right) K_{1}\left(\eta^{\prime}, \mu\right)^{-1} \operatorname{diag}\left(\rho^{-\sigma_{1}}, \ldots, \rho^{-\sigma_{N_{r}}}\right),\right.
\end{aligned}
$$

where $K_{3}\left(\eta^{\prime}, \mu, \rho\right)$ is an $N_{r} \times N_{r}$ matrix function defined by

$$
I_{N_{r}}+K_{3}\left(\eta^{\prime}, \mu, \rho\right)=\left(I_{N_{r}}+K_{1}\left(\eta^{\prime}, \mu\right)^{-1} K_{2}\left(\eta^{\prime}, \mu, \rho\right)\right)^{-1}
$$


and where each entry of $K_{3}\left(\eta^{\prime}, \mu, \rho\right)$ is a function of its arguments of rational type and is bounded in modulus by the expression on the right-hand side of (3.20). Hence it follows that

$$
\begin{aligned}
& \int_{\gamma_{r}^{+}\left(\xi^{\prime}, \lambda\right)} \mathcal{B}^{(r, r)}\left(0, \xi^{\prime}, z\right)\left(\AA\left(0, \xi^{\prime}, z\right)-\lambda I_{N}\right)^{-1}\left(\begin{array}{c}
\widetilde{G}^{(1)}\left(\eta^{\prime}, \zeta, \mu, \rho\right)+\widetilde{G}^{(2)}\left(\eta^{\prime}, \zeta, \mu, \rho\right) \\
0 I_{N-k_{r}}
\end{array}\right) d z \\
& \quad=I_{N_{r}},
\end{aligned}
$$

where

$$
\begin{aligned}
& \widetilde{G}^{(1)}\left(\eta^{\prime}, \zeta, \mu, \rho\right)= \rho^{-1} \operatorname{diag}\left(\rho^{s_{1}}, \ldots, \rho^{s_{k_{r}}}\right) J Z(\zeta) K_{1}\left(\eta^{\prime}, \mu\right)^{-1} \operatorname{diag}\left(\rho^{-\sigma_{1}}, \ldots, \rho^{-\sigma_{N_{r}}}\right), \\
& \widetilde{G}^{(2)}\left(\eta^{\prime}, \zeta, \mu, \rho\right)=\rho^{-1} \operatorname{diag}\left(\rho^{s_{1}}, \ldots, \rho^{s_{k_{r}}}\right) J Z(\zeta) K_{3}\left(\eta^{\prime}, \mu, \rho\right) K_{1}\left(\eta^{\prime}, \mu\right)^{-1} \\
& \quad \times \operatorname{diag}\left(\rho^{-\sigma_{1}}, \ldots, \rho^{-\sigma_{N_{r}}}\right) .
\end{aligned}
$$

Suppose next that $r<\ell \leq d$ and let $\Lambda^{(\ell)}\left(\xi^{\prime}, \lambda\right)$ denote the expression (3.27). Then we know that

$$
\begin{aligned}
& \Lambda^{(\ell)}\left(\xi^{\prime}, \lambda\right)= \rho_{\ell} \\
& \operatorname{diag}\left(\rho_{\ell}^{\sigma_{N_{\ell-1}+1}}, \ldots, \rho_{\ell}^{\sigma_{N_{\ell}}}\right)\left(K_{1}^{(\ell)}\left(\mu_{\ell}\right)+K_{2}^{(\ell)}\left(\eta_{\ell}^{\prime}, \mu_{\ell}, \rho_{\ell}\right)\right) \\
& \times \operatorname{diag}\left(\rho_{\ell}^{q(1)-s_{k(1)}}, \ldots, \rho_{\ell}^{\left.q\left(N_{\ell}-N_{\ell-1}\right)-s_{k\left(N_{\ell}-N_{\ell-1}\right)}\right),}\right.
\end{aligned}
$$

where $K_{2}^{(\ell)}\left(\eta_{\ell}^{\prime}, \mu_{\ell}, \rho_{\ell}\right)=\int_{\gamma_{r, \ell}^{+}} \widetilde{K}_{2}^{(\ell)}\left(\eta_{\ell}, \zeta_{\ell}, \mu_{\ell}, \rho_{\ell}\right) J^{(\ell)} Z^{(\ell)}\left(\zeta_{\ell}\right) d \zeta_{\ell}$. Hence bearing in mind (3.24) and (3.28), it follows that $\Lambda^{(\ell)}\left(\xi^{\prime}, \lambda\right)$ is invertible and

$$
\begin{aligned}
& \Lambda^{(\ell)}\left(\xi^{\prime}, \lambda\right)^{-1}=\rho_{\ell}^{-1} \operatorname{diag}\left(\rho^{s(1)-q(1)}, \ldots, \rho_{\ell}^{s_{k\left(N_{\ell}-N_{\ell-1}\right)}-q\left(N_{\ell}-N_{\ell-1}\right)}\right) \\
& \quad \times\left(I_{N_{\ell}-N_{\ell-1}}+K_{3}^{(\ell)}\left(\eta_{\ell}^{\prime}, \mu_{\ell}, \rho_{\ell}\right)\right) K_{1}^{(\ell)}\left(\mu_{\ell}\right)^{-1} \operatorname{diag}\left(\rho_{\ell}^{-\sigma_{N_{\ell-1}+1}}, \ldots, \rho^{-\sigma_{N_{\ell}}}\right),
\end{aligned}
$$

where $K_{3}^{(\ell)}\left(\eta_{\ell}^{\prime}, \mu_{\ell}, \rho_{\ell}\right)$ is an $\left(N_{\ell}-N_{\ell-1}\right) \times\left(N_{\ell}-N_{\ell-1}\right)$ matrix function defined by

$$
I_{N_{\ell}-N_{\ell-1}}+K_{3}^{(\ell)}\left(\eta_{\ell}^{\prime}, \mu_{\ell}, \rho_{\ell}\right)=\left(I_{N_{\ell}-N_{\ell-1}}+K_{1}^{(\ell)}\left(\mu_{\ell}\right)^{-1} K_{2}^{(\ell)}\left(\eta_{\ell}^{\prime}, \mu_{\ell}, \rho_{\ell}\right)\right)^{-1}
$$

and each entry of $K_{3}^{(\ell)}\left(\eta_{\ell}^{\prime}, \mu_{\ell}, \rho_{\ell}\right)$ is a function of its arguments of rational type and is bounded in modulus by the expression on the right-hand side of (3.28). Hence it follows that

$$
\begin{aligned}
& \int_{\gamma_{r, \ell}^{+}(\lambda)} \mathcal{B}^{(r, \ell)}\left(0, \xi^{\prime}, z\right)\left(\AA\left(0, \xi^{\prime}, z\right)-\lambda I_{N}\right)^{-1}\left(\widetilde{G}^{(1, \ell)}\left(\zeta_{\ell}, \mu_{\ell}, \rho_{\ell}\right)+\widetilde{G}^{(2, \ell)}\left(\eta_{\ell}^{\prime}, \zeta_{\ell}, \mu_{\ell}, \rho_{\ell}\right)\right) d z \\
& \quad=I_{N_{\ell}-N_{\ell-1}}
\end{aligned}
$$

where

$$
\begin{aligned}
\widetilde{G}^{(1, \ell)}\left(\zeta_{\ell}, \mu_{\ell}, \rho_{\ell}\right) & =\rho_{\ell}^{-1} \operatorname{diag}\left(\rho_{\ell}^{s_{1}}, \ldots, \rho_{\ell}^{s_{k_{\ell}}}\right) J^{(\ell)} Z^{(\ell)}\left(\zeta_{\ell}\right) K_{1}^{(\ell)}\left(\mu_{\ell}\right)^{-1} \\
& \times \operatorname{diag}\left(\rho_{\ell}^{-\sigma_{N_{\ell-1}+1}}, \ldots, \rho_{\ell}^{-\sigma_{N_{\ell}}}\right), \\
\widetilde{G}^{(2, \ell)}\left(\eta_{\ell}^{\prime}, \zeta_{\ell}, \mu_{\ell}, \rho_{\ell}\right) & =\rho_{\ell}^{-1} \operatorname{diag}\left(\rho_{\ell}^{s_{1}}, \ldots, \rho_{\ell}^{s_{k_{\ell}}}\right) J^{(\ell)} Z^{(\ell)}\left(\zeta_{\ell}\right) K_{3}^{(\ell)}\left(\eta_{\ell}^{\prime}, \zeta_{\ell}, \mu_{\ell}, \rho_{\ell}\right) \\
& \times K_{1}^{(\ell)}\left(\mu_{\ell}\right)^{-1} \operatorname{diag}\left(\rho_{\ell}^{-\sigma_{N_{\ell-1}+1}}, \ldots, \rho_{\ell}^{-\sigma_{N_{\ell}}}\right) .
\end{aligned}
$$

Finally if we rewrite $\rho, \eta^{\prime}, \zeta$, and $\mu$ as $\rho_{r, r}, \eta_{r, r}^{\prime}, \zeta_{r, r}$, and $\mu_{r, r}$, respectively, and put

$$
K^{r, r}\left(\eta_{r, r}^{\prime}, \zeta_{r, r}, \mu_{r, r}, \rho_{r, r}\right)=J Z\left(\zeta_{r, r}\right)\left(I_{N_{r}}+K_{3}\left(\eta_{r, r}^{\prime}, \mu_{r, r}, \rho_{r, r}\right)\right) K_{1}\left(\eta_{r, r}^{\prime}, \mu_{r, r}\right)^{-1},
$$


while if for $\ell>r$ we rewrite $\rho_{\ell}, \eta_{\ell}^{\prime}, \zeta_{\ell}$, and $\mu_{\ell}$ as $\rho_{r, \ell}, \eta_{r, \ell}^{\prime}, \zeta_{r, \ell}$, and $\mu_{r, \ell}$, respectively, and put

$$
\begin{aligned}
& K^{(r, \ell)}\left(\eta_{r, \ell}^{\prime}, \zeta_{r, \ell}, \mu_{r, \ell}, \rho_{r, \ell}\right) \\
& \quad=J^{(\ell)} Z^{(\ell)}\left(\zeta_{r, \ell}\right)\left(I_{N_{\ell}-N_{\ell-1}}+K_{3}^{(\ell)}\left(\eta_{r, \ell}^{\prime}, \mu_{r, \ell}, \rho_{r, \ell}\right)\right) K_{1}^{(\ell)}\left(\mu_{r, \ell}\right)^{-1},
\end{aligned}
$$

then all the assertions of the proposition for the case under consideration here follow from the foregoing results.

Let us fix $r, 0 \leq r<d$, and referring again to Proposition 3.12 for notation, let us denote by $\bar{W}^{(r)}\left(\xi^{\prime}, x_{n}, \lambda\right)$ the $N \times N_{d}$ matrix function whose columns are precisely the $w^{(r, \nu)}\left(\xi^{\prime}, x_{n}, \lambda\right)$. For $r^{\dagger} \leq j \leq d$, let

$$
\begin{aligned}
& \mathcal{I}_{r, j}\left(\xi^{\prime}, \lambda\right)=\left(\mathcal{B}^{\left(r^{\dagger}, \ell\right)}\left(0, \xi^{\prime}, D_{n}\right) W^{\left(r, \ell_{1}\right)}\left(\xi^{\prime}, 0, \lambda\right)\right)_{\ell, \ell_{1}=j}^{d}, \\
& \widetilde{\mathcal{I}}_{r, j}\left(\xi^{\prime}, \lambda\right)=\left(\widetilde{\mathcal{B}}^{\left(r^{\dagger}, \ell\right)}\left(0, \xi^{\prime}, D_{n}\right) \widetilde{W}^{\left(r, \ell_{1}\right)}\left(\xi^{\prime}, 0, \lambda\right)\right)_{\ell, \ell_{1}=j}^{d},
\end{aligned}
$$

where

$$
\begin{gathered}
\widetilde{\mathcal{B}}^{\left(r^{\dagger}, \ell\right)}\left(0, \xi^{\prime}, D_{n}\right)=\operatorname{diag}\left(\rho_{r, \ell}^{-\sigma_{N_{r, \ell-1}^{\dagger}+1}^{\dagger}}, \ldots, \rho_{r, \ell}^{-\sigma_{N_{\ell}}}\right) \mathcal{B}^{\left(r^{\dagger}, \ell\right)}\left(0, \xi^{\prime}, D_{n}\right), \\
\widetilde{W}^{\left(r, \ell_{1}\right)}\left(\xi^{\prime}, 0, \lambda\right)=W^{\left(r, \ell_{1}\right)}\left(\xi^{\prime}, 0, \lambda\right) \operatorname{diag}\left(\rho_{r, \ell_{1}}^{\sigma_{r, \ell_{1}-1}^{+1}}, \ldots, \rho_{r, \ell_{1}}^{\sigma_{\ell_{\ell_{1}}}}\right) .
\end{gathered}
$$

Then we can write

$$
\begin{aligned}
& \stackrel{\circ}{B}\left(0, \xi^{\prime}, D_{n}\right) W^{(r)}\left(\xi^{\prime}, 0, \lambda\right)=\mathcal{I}_{r, r^{\dagger}}\left(\xi^{\prime}, \lambda\right) \\
&=\operatorname{diag}\left(\widetilde{\rho}_{1}^{\sigma_{1}}, \ldots, \widetilde{\rho}_{N_{d}}^{\sigma_{N_{d}}}\right) \widetilde{\mathcal{I}}_{r, r^{\dagger}}\left(\xi^{\prime}, \lambda\right) \operatorname{diag}\left(\widetilde{\rho}_{1}^{-\sigma_{1}}, \ldots, \widetilde{\rho}_{N_{d}}^{-\sigma_{N_{d}}}\right),
\end{aligned}
$$

where

$$
\widetilde{\rho}_{\nu}= \begin{cases}\rho_{r, r^{\dagger}} & \text { for } 1 \leq \nu \leq N_{r^{\dagger}} \\ \rho_{r, \ell} & \text { for } N_{\ell-1}<\nu \leq N_{\ell}, \ell=r^{\dagger}+1, \ldots, d\end{cases}
$$

We remark at this point that as a consequence of Proposition 3.14 below, it will be seen that for $\ell \neq \ell_{1}, \widetilde{\mathcal{B}}^{\left(r^{\dagger}, \ell\right)}\left(0, \xi^{\prime}, D_{n}\right) \widetilde{W}^{\left(r, \ell_{1}\right)}\left(\xi^{\prime}, 0, \lambda\right)$ is a $\left(N_{\ell}-N_{r, \ell-1}^{\dagger}\right) \times\left(N_{\ell_{1}}-\right.$ $\left.N_{r, \ell_{1}-1}^{\dagger}\right)$ matrix function whose entries are products of powers of $\rho_{r, \ell} / \rho_{r, \ell_{1}}$ and an expression of rational type in the components of $\eta_{r, \ell_{1}}^{\prime}, \mu_{r, \ell_{1}}$, and $\rho_{r, \ell_{1}}$.

Definition 3.13. Suppose that the boundary problem (1.1), (1.2) is parameterelliptic in $\mathcal{L}$ and that $\lambda \in \mathcal{L}$. Suppose also that $x^{0} \in \Gamma$ and that the boundary problem has been rewritten in terms of a fixed local coordinate system at $x^{0}$ (see the paragraph preceding Definition 2.3 and $[\mathrm{AV}$, p. 63]). In addition, suppose that with respect to this boundary problem all hypotheses of Proposition 3.12 hold. Then bearing in mind the definitions of the various terms introduced above, we say that the operators $A(x, D)$ and $B(x, D)=\left(B_{1}(x, D), \ldots, B_{\widetilde{N}}(x, D)\right)^{T}$ are compatible at $x^{0}$ if for each $r$ satisfying $0 \leq r<d$ and for each pair of integers $\ell, \ell_{1}$ satisfying $r^{\dagger} \leq \ell_{1}<d, \ell_{1}+1 \leq \ell \leq d$, each entry of the matrix $\widetilde{\mathcal{B}}^{(r, \ell)}\left(0, \xi^{\prime}, D_{n}\right) \widetilde{W}^{\left(r, \ell_{1}\right)}\left(\xi^{\prime}, 0, \lambda\right)$ is bounded in modulus by a constant depending only upon $\left(\eta_{r, r^{\dagger}}^{\prime}, \mu_{r, r^{\dagger}}\right) \in \Sigma_{r}$ and $\mu_{r, \ell} \in \mathcal{L}_{1}$ for $\ell>r$.

Proposition 3.14. Suppose that the hypotheses of Proposition 3.12 hold and that the operators $A(x, D)$ and $B(x, D)$ are compatible at $x^{0}$. Suppose also that $0 \leq$ $r<d$. Then for $\epsilon^{0}$ sufficiently small and $\lambda^{0}$ sufficiently large the matrix function 
$\widetilde{\mathcal{I}}_{r, r^{\dagger}}\left(\xi^{\prime}, \lambda\right)$ of (3.33), and hence the matrix function $\mathcal{I}_{r, r^{\dagger}}\left(\xi^{\prime}, \lambda\right)$ are invertible and we have

$$
\mathcal{I}_{r, r^{\dagger}}\left(\xi^{\prime}, \lambda\right)^{-1}=\operatorname{diag}\left(\widetilde{\rho}_{1}^{\sigma_{1}}, \ldots, \widetilde{\rho}_{N_{d}}^{\sigma_{N_{d}}}\right) \widetilde{\mathcal{I}}_{r, r^{\dagger}}\left(\xi^{\prime}, \lambda\right)^{-1} \operatorname{diag}\left(\widetilde{\rho}_{1}^{-\sigma_{1}}, \ldots, \widetilde{\rho}_{N_{d}}^{-\sigma_{N_{d}}}\right),
$$

where $\left|\operatorname{det} \widetilde{\mathcal{I}}_{r, r^{\dagger}}\left(\xi^{\prime}, \lambda\right)\right|>\frac{1}{2}$, while the entries of $\widetilde{\mathcal{I}}_{r, r^{\dagger}}\left(\xi^{\prime}, \lambda\right)^{-1}$ are rational functions of the $\rho_{r, \ell}$ and expressions of rational type in $\eta_{r, \ell}^{\prime}, \mu_{r, \ell}$, and $\rho_{r, \ell}$ for $\ell \geq r^{\dagger}$ and are bounded in modulus by a constant depending only upon $\left(\eta_{r, r^{\dagger}}^{\prime}, \mu_{r, r^{\dagger}}\right) \in \Sigma_{r}$ and $\mu_{r, \ell} \in$ $\mathcal{L}_{1}$ for $\ell>r$. Lastly, the operators $A(x, D)$ and $B(x, D)$ are always compatible at $x^{0}$ if

(i) the boundary conditions (1.2) are of Dirichlet type at $x^{0}$ or if

(ii) the operators $A(x, D)$ and $B(x, D)$ are both essentially upper triangular at $x^{0}$.

Proof. We will only prove the proposition for the case $1 \leq r<d$; the case $r=0$ can be similarly treated. Accordingly, let us fix our attention upon (3.32) and suppose that $\ell \neq \ell_{1}$.

Then it follows from the proofs of Proposition 3.11 and 3.12 that

$$
\begin{aligned}
\widetilde{\mathcal{B}}^{(r, \ell)}\left(0, \xi^{\prime}, D_{n}\right) \widetilde{W}^{(r, \ell)}\left(\xi^{\prime}, 0, \lambda\right)=\operatorname{diag}\left(\left(\frac{\rho_{r, \ell}}{\rho_{r, \ell_{1}}}\right)^{-\sigma_{N_{r, \ell-1}+1}^{\dagger}}, \ldots,\left(\frac{\rho_{r, \ell}}{\rho_{r, \ell_{1}}}\right)^{-\sigma_{N_{\ell}}}\right) \\
\times \int_{\widetilde{\gamma}_{r, \ell_{1}}}\left[\mathcal{B}_{\ell_{1}, 1}^{(r, \ell)}\left(\eta_{r, \ell_{1}}^{\prime}, \zeta_{r, \ell_{1}}\right)\left(\widetilde{a}_{j k}^{\left(r, \ell_{1}\right)}\left(\eta_{r, \ell_{1}}^{\prime}, \zeta_{r, \ell_{1}}, \mu_{r, \ell_{1}}\right)\right)_{j, k=1}^{k_{\ell_{1}}}\right. \\
\times\left(I_{k_{\ell_{1}}}+H_{11}^{\sharp\left(r, \ell_{1}\right)}\left(\eta_{r, \ell_{1}}^{\prime}, \zeta_{r, \ell_{1}}, \mu_{r, \ell_{1}}, \rho_{r, \ell_{1}}\right)\right) \\
-\left(\mu_{r, \ell_{1}} \rho_{r, \ell_{1}}^{\widetilde{m}_{\ell_{1}}}\right)^{-1} \mathcal{B}_{\ell_{1}, 2}^{(r, \ell)}\left(\eta_{r, \ell_{1}}^{\prime}, \zeta_{r, \ell_{1}}\right) \operatorname{diag}\left(\rho_{r, \ell_{1}+1}^{m_{\ell_{1}+1}}, \ldots, \rho_{r, \ell_{1}}^{m_{N}}\right) \\
\left.\times H_{21}^{\sharp\left(r, \ell_{1}\right)}\left(\eta_{r, \ell_{1}}^{\prime}, \zeta_{r, \ell_{1}}, \mu_{r, \ell_{1}}, \rho_{r, \ell_{1}}\right)\right] K^{\left(r, \ell_{1}\right)}\left(\eta_{r, \ell_{1}}^{\prime}, \zeta_{r, \ell_{1}}, \mu_{r, \ell_{1}}, \rho_{r, \ell_{1}}\right) d \zeta_{r, \ell_{1}},
\end{aligned}
$$

where, referring to Proposition 3.11 for notation, $\widetilde{\gamma}_{r, \ell_{1}}^{+}$denotes $\gamma_{r}^{+}$if $\ell_{1}=r$ and $\gamma_{r, \ell_{1}}^{+}$ otherwise, $\widetilde{a}_{j k}^{\left(r, \ell_{1}\right)}\left(\eta_{r, \ell_{1}}^{\prime}, \zeta_{r, \ell_{1}}, \mu_{r, \ell_{1}}\right)$ denotes $\widetilde{a}_{j k}\left(\eta^{\prime}, \zeta, \mu\right)$ if $\ell_{1}=r$ and $\widetilde{a}_{j k}^{\left(\ell_{1}\right)}\left(\zeta_{\ell_{1}}, \mu_{\ell_{1}}\right)$ otherwise, and $H_{j k}^{\sharp\left(r, \ell_{1}\right)}\left(\eta_{r, \ell_{1}}^{\prime}, \zeta_{r, \ell_{1}}, \mu_{r, \ell_{1}}, \rho_{r, \ell_{1}}\right)$ denotes $H_{j k}^{\sharp}\left(\eta^{\prime}, \zeta, \mu, \rho\right)$ if $\ell_{1}=r$ and $H_{j k}^{\sharp\left(\ell_{1}\right)}\left(\eta_{\ell_{1}}^{\prime}, \zeta_{\ell_{1}}, \mu_{\ell_{1}}, \rho_{\ell_{1}}\right)$ otherwise.

Suppose that $\ell>\ell_{1}$. Then by hypothesis the entries of $\widetilde{\mathcal{B}}^{(r, \ell)}\left(0, \xi^{\prime}, D_{n}\right)$

$\widetilde{W}^{\left(r, \ell_{1}\right)}\left(\xi^{\prime}, 0, \lambda\right)$ are bounded in modulus by a constant depending only on $\left(\eta_{r, r}^{\prime}, \mu_{r, \ell}\right)$ $\in \Sigma_{r}$ and $\mu_{r, \ell} \in \mathcal{L}_{1}$ for $\ell>r$. Let us now show that this boundedness condition is always satisfied if the boundary conditions (1.2) are of Dirichlet type at $x^{0}$. Indeed, if this latter condition holds, then every entry of $\mathcal{B}_{\ell_{1}, 1}^{(r, \ell)}\left(\eta_{r, \ell_{1}}^{\prime}, \zeta_{r, \ell_{1}}\right)$ is 0 , while the only non-zero entries of $\mathcal{B}_{\ell_{1}, 2}^{(r, \ell)}\left(\eta_{r, \ell_{1}}^{\prime}, \zeta_{r, \ell_{1}}\right)$ are those lying in rows $N_{\ell}-\nu$, $\nu=0, \ldots, N_{\ell}-N_{\ell-1}-1$, and columns $k_{\ell}-\widetilde{\nu}, \widetilde{\nu}=0, \ldots, k_{\ell}-k_{\ell-1}-1$. Then recalling from Section 1 that we now have $s_{j}=t_{j}=t_{j}^{\prime}$ for $j=1, \ldots, N$, it follows from the foregoing results and (3.34) that the entry in the $\left(N_{\ell}-\nu\right)$-th row and the $\left(k_{\ell}-\widetilde{\nu}\right)$-th column of $\widetilde{\mathcal{B}}^{(r, \ell)}\left(0, \xi^{\prime}, D_{n}\right) \widetilde{W}^{\left(r, \ell_{1}\right)}\left(\xi^{\prime}, 0, \lambda\right)$ is bounded in modulus by

$$
C|\lambda|^{-\frac{1}{2}-\frac{t_{k_{\ell}}^{\prime}}{t_{k_{\ell_{1}}}^{\prime}}-\frac{\nu^{\dagger}}{2}\left(\frac{1}{t_{k_{\ell}}^{\prime}}-\frac{1}{t_{k_{\ell_{1}}}^{\prime}}\right)}\left(|\lambda|^{\frac{t_{t_{\ell_{\ell}}}^{\prime}}{t_{\ell_{\ell_{1}}}^{\prime}}}+\delta_{r, \ell_{1}} \epsilon_{r+1}\right)
$$


where $0 \leq \nu^{\dagger} \leq t_{k_{\ell}}^{\prime}-1$ and the constant $C$ depends only upon $\left(\eta_{r, r}^{\prime}, \mu_{r, r}\right) \in \Sigma_{r}$ and $\mu_{r, \ell} \in \mathcal{L}_{1}$. Our claim concerning Dirichlet boundary conditions are an immediate consequence of this last result.

Note also from (3.34) that when $A(x, D)$ and $B(x, D)$ are essentially upper triangular at $x^{0}$, then every entry of $\mathcal{B}_{\ell_{1}, 1}^{(r, \ell)}\left(\eta_{r, \ell_{1}}^{\prime}, \zeta_{r, \ell_{1}}\right)$ (resp. every entry of $\left.H_{21}^{\sharp\left(r, \ell_{1}\right)}\left(\eta_{r, \ell_{1}}^{\prime}, \zeta_{r, \ell_{1}}, \mu_{r, \ell_{1}}, \rho_{r, \ell_{1}}\right)\right)$ is 0 , and hence the entries of $\widetilde{\mathcal{B}}^{(r, \ell)}\left(0, \xi^{\prime}, D_{n}\right)$ $\widetilde{W}^{\left(r, \ell_{1}\right)}\left(\xi^{\prime}, 0, \lambda\right)$ are all 0 . Thus the boundedness condition also holds under the cited conditions concerning $A(x, D)$ and $B(x, D)$.

Let us now consider the case $\ell_{1}>\ell$. Then it is a simple matter to deduce that for this case each entry of (3.34) is bounded in modulus by

$$
C\left(|\lambda|^{\frac{1}{\bar{m}_{\ell}}-\frac{1}{m_{\ell_{1}}}}+\delta_{r+1, \ell_{1}} \epsilon_{r+1}+\left(1-\delta_{\ell_{1}, d}\right)|\lambda|^{\frac{\widetilde{m}_{\ell_{1}}+1}{m_{\ell_{1}}}-1}\right),
$$

where the constant $C$ depends only upon $\mu_{r, \ell} \in \mathcal{L}_{1}$.

Returning again to (3.32), let us next introduce the block row and column matrices

$$
\begin{aligned}
& \widetilde{\mathcal{I}}_{r, j}^{(1,2)}\left(\xi^{\prime}, \lambda\right)=\left(\widetilde{\mathcal{B}}^{(r, j)}\left(0, \xi^{\prime}, D_{n}\right) \widetilde{W}^{\left(r, \ell_{1}\right)}\left(\xi^{\prime}, 0, \lambda\right)\right)_{\ell_{1}=j+1}^{d}, \\
& \widetilde{\mathcal{I}}_{r, j}^{(2,1)}\left(\xi^{\prime}, \lambda\right)=\left(\widetilde{\mathcal{B}}^{(r, \ell)}\left(0, \xi^{\prime}, D_{n}\right) \widetilde{W}^{(r, j)}\left(\xi^{\prime}, 0, \lambda\right)\right)_{\ell=j+1}^{d},
\end{aligned}
$$

respectively. Then a factorization in the sense of Schur gives

$$
\begin{aligned}
\widetilde{\mathcal{I}}_{r, j}\left(\xi^{\prime}, \lambda\right) & =\left(\begin{array}{cc}
I_{N_{j}-N_{r, j-1}^{\dagger}} & 0 \\
\widetilde{\mathcal{I}}_{r, j}^{(2,1)}\left(\xi^{\prime}, \lambda\right) & I_{N_{d}-N_{j}}
\end{array}\right) \\
& \times\left(\begin{array}{cc}
I_{N_{j}-N_{r, j-1}^{\dagger}} & \widetilde{\mathcal{I}}_{r, j+1}\left(\xi^{\prime}, \lambda\right)-\widetilde{\mathcal{I}}_{r, j}^{(2,1)}\left(\xi^{\prime}, \lambda\right) \widetilde{\mathcal{I}}_{r, j}^{(1,2)}\left(\xi^{\prime}, \lambda\right) \\
0 & \widetilde{\mathcal{I}}_{r, j}^{(1,2)}\left(\xi^{\prime}, \lambda\right)
\end{array}\right) . \\
& \times\left(\begin{array}{cc}
I_{N_{j}-N_{r, j-1}^{\dagger}} & I_{N_{d}-N_{j}}
\end{array}\right) .
\end{aligned}
$$

Since we already know that each entry of $\widetilde{\mathcal{I}}_{r, j}^{(2,1)}\left(\xi^{\prime}, \lambda\right)$ is bounded in modulus by a constant depending only upon $\left(\eta_{r, r}^{\prime}, \mu_{r, r}\right) \in \Sigma_{r}$, that each entry of $\widetilde{\mathcal{I}}_{r, j}^{(1,2)}\left(\xi^{\prime}, \lambda\right)$ is bounded in modulus by the quantity (3.35), and that $\widetilde{\mathcal{I}}_{r, d}\left(\xi^{\prime}, \lambda\right)=I_{N_{d}-N_{r, d-1}^{\dagger}}$, an inductive argument involving Schur factorizations shows that we may choose $\epsilon^{0}$ sufficiently small and $\lambda^{0}$ sufficiently large so that all the assertions of the proposition concerning $\widetilde{\mathcal{I}}_{r, r}\left(\xi^{\prime}, \lambda\right)$ hold. This completes the proof of the proposition for the case under consideration.

As a consequence of Proposition 3.14, we are now in a position to present the last result required for the proof of Proposition 3.6. To this end we require some further notation. Accordingly, for $0 \leq r \leq d$ and $\lambda \in \mathbb{C} \backslash\{0\}$ let $\chi_{\lambda}^{(r)} \in C^{\infty}\left(\mathbb{R}^{n-1}\right)$ such that $0 \leq \chi_{\lambda}^{(r)}\left(\xi^{\prime}\right) \leq 1, \chi_{\lambda}^{(r)}=1$ for $0 \leq\left|\xi^{\prime}\right| \leq \frac{3}{4} \epsilon_{1}|\lambda|^{1 / \tilde{m}_{1}}$ and vanishes for $\left|\xi^{\prime}\right| \geq \frac{7}{8} \epsilon_{1}|\lambda|^{1 / \widetilde{m}_{1}}$ if $r=0, \chi_{\lambda}^{(r)}\left(\xi^{\prime}\right)=1$ for $\frac{1}{4} \epsilon_{r}|\lambda|^{1 / \widetilde{m}_{r}} \leq\left|\xi^{\prime}\right| \leq \frac{3}{4} \epsilon_{r+1}|\lambda|^{1 / \widetilde{m}_{r+1}}$ and vanishes for $\left|\xi^{\prime}\right| \leq \frac{1}{8} \epsilon_{r}|\lambda|^{1 / \widetilde{m}_{r}}$ and for $\left|\xi^{\prime}\right| \geq \frac{7}{8} \epsilon_{r+1}|\lambda|^{1 / \tilde{m}_{r+1}}$ if $1 \leq r<d, \chi_{\lambda}^{(r)}\left(\xi^{\prime}\right)=1$ for $\frac{1}{4} \epsilon_{d}|\lambda|^{1 / \widetilde{m}_{d}} \leq\left|\xi^{\prime}\right|<\infty$ and vanishes for $\left|\xi^{\prime}\right| \leq \frac{1}{8} \epsilon_{d}|\lambda|^{1 / \widetilde{m}_{d}}$ if $r=d$, while $\chi_{\lambda}^{(r)}\left(\xi^{\prime}\right)$ is a Fourier multiplier of type $(p, p)$ whose norm is bounded by a constant not 
depending upon $\lambda, \epsilon_{r}$, and $\epsilon_{r+1}$. We also let $\mathcal{S}\left(\mathbb{R}^{n-1}\right)$ denote the Schwartz space of rapidly decreasing functions on $\mathbb{R}^{n-1}$ and $F^{\prime}$ the Fourier transform in $\mathbb{R}^{n-1}$ $\left(x^{\prime} \mapsto \xi^{\prime}\right)$.

Proposition 3.15. Suppose that the boundary problem (1.1)-(1.2) is parameterelliptic in $\mathcal{L}$. Suppose that $x^{0} \in \Gamma$ and that the boundary problem (1.1), (1.2) is rewritten in terms of the local coordinates at $x^{0}$. In addition, suppose that the operators $A(x, D)$ and $B(x, D)$ are compatible at $x^{0}$ and for $g \in \mathcal{S}\left(\mathbb{R}^{n-1}\right)^{\tilde{N}}$ and $0 \leq r \leq d$, let $g^{(r)}\left(x^{\prime}\right)=\left(F^{\prime-1} \psi_{r}\left(\xi^{\prime}\right) F^{\prime} g\right)\left(x^{\prime}\right)$. Then there exists a $\lambda_{0}=\lambda_{0}(p)>0$ such that for $\lambda \in \mathcal{L}$ with $|\lambda| \geq \lambda_{0}$, the boundary problem

$$
\begin{aligned}
\stackrel{\circ}{A}(0, D) u(x)-\lambda u(x) & =0 \quad \text { for } x_{n}>0, \\
\stackrel{\circ}{B}(0, D) u(x) & =g^{(r)}\left(x^{\prime}\right) \quad \text { at } x_{n}=0,
\end{aligned}
$$

has a solution $u^{(r)}(x)=\left(u_{1}^{(r)}(x), \ldots, u_{N}^{(r)}(x)\right)^{T} \in \prod_{j=1}^{N} W_{p}^{t_{j}}\left(\mathbb{R}_{+}^{n}\right)$ and the a priori estimate

$$
\sum_{j=1}^{N}\left\|u_{j}^{(r)}\right\|_{t_{j}, p, \mathbb{R}_{+}^{n}}^{\left(\pi_{1}(j)\right)} \leq C \sum_{j=1}^{\widetilde{N}}\left\|g_{j}^{(r)}\right\|_{-\sigma_{j}-\frac{1}{p}, p, \mathbb{R}^{n-1}}^{\left(\pi_{2}(j)\right)}
$$

holds, where the constant $C$ does not depend upon $g$ and $\lambda$.

Proof. We will only prove the proposition for the case $1 \leq r<d$; the remaining cases can be treated similarly. Accordingly, under Fourier transform with respect to $x^{\prime}$, the boundary problem $(3.36),(3.37)$ is transformed into the boundary problem: $(3.8)$,

$$
\stackrel{\circ}{B}\left(0, \xi^{\prime}, D_{n}\right) F^{\prime} u\left(\xi^{\prime}, x_{n}\right)=F^{\prime} g^{(r)}\left(\xi^{\prime}\right) \quad \text { at } x_{n}=0 .
$$

Then assuming that $\epsilon^{0}$ and $\lambda^{0}$ have been chosen so that the conclusions of Propositions 3.12 and 3.14 hold, it follows from these propositions that for a fixed $\lambda \in \mathcal{L}$ with $|\lambda| \geq \lambda_{0}$, this boundary problem has a solution in the space of vector functions decaying exponentially at $\infty, \widetilde{u}^{(r)}\left(\xi^{\prime}, x_{n}, \lambda\right)$, which is unique for $\frac{1}{8} \epsilon_{r}|\lambda|^{1 / \widetilde{m}_{r}} \leq\left|\xi^{\prime}\right| \leq \frac{7}{8} \epsilon_{r+1}|\lambda|^{1 / \widetilde{m}_{r+1}}$ and is given in block row matrix form by

$$
\begin{aligned}
\widetilde{u}^{(r)}\left(\xi^{\prime}, x_{n}, \lambda\right)= & \sum_{\ell=r}^{d} \operatorname{diag}\left(\rho_{r, \ell}^{-t_{1}}, \ldots, \rho_{r, \ell}^{-t_{N}}\right) \\
& \times\left(\int_{\gamma_{r, \ell}^{+}} e^{i \rho_{r, \ell} x_{n} \zeta_{r, \ell}} \mathcal{G}^{(r, \ell)}\left(\underline{\eta}_{r}^{\prime}, \zeta_{r, \ell}, \underline{\mu}_{r}, \underline{\rho}_{r}\right) d \zeta_{r, \ell}\right) \\
& \times \operatorname{diag}\left(\widetilde{\rho}_{1}^{-\sigma_{1}}, \ldots, \widetilde{\rho}_{N_{d}}^{-\sigma_{N_{d}}}\right) g^{(r)}\left(\xi^{\prime}\right),
\end{aligned}
$$

where $\underline{\eta}_{r}^{\prime}=\left(\eta_{r, r}^{\prime}, \ldots, \eta_{r, d}^{\prime}\right), \underline{\mu}_{r}=\left(\mu_{r, r}, \ldots, \mu_{r, d}\right), \underline{\rho}_{r}=\left(\rho_{r, r}, \ldots, \rho_{r, d}\right)$,

$$
\begin{aligned}
\mathcal{G}^{(r, \ell)}\left(\underline{\eta}_{r}^{\prime}, \zeta_{r, \ell}, \underline{\mu}_{r}, \underline{\rho}_{r}\right)= & \left(\begin{array}{c}
\left(\widetilde{a}_{j, k}^{(r, \ell)}\left(\eta_{r, \ell}^{\prime}, \zeta_{r, \ell}, \mu_{r, \ell}\right)\right)_{j, k=1}^{k_{\ell}}\left(I_{k_{\ell}}+H_{11}^{\sharp(r, \ell)}(\cdot) K^{(r, \ell)}(\cdot)\right) \\
-\lambda^{-1} H_{21}^{\sharp(r, \ell)}(\cdot) K^{(r, \ell)}(\cdot)
\end{array}\right) \\
& \times \widetilde{\mathcal{I}}_{r, r}^{(\ell)}\left(\underline{\eta}_{r}^{\prime}, \underline{\mu}_{r}, \underline{\rho}_{r}\right)^{-1},
\end{aligned}
$$

$\widetilde{\mathcal{I}}_{r, r}^{(\ell)}\left(\underline{\eta}_{r}^{\prime}, \underline{\mu}_{r}, \underline{\rho}_{r}\right)^{-1}$ denotes the matrix whose rows are precisely rows $N_{r, \ell-1}^{\dagger}+1$ to $N_{r, \ell}$ of $\tilde{\mathcal{I}}_{r, r}\left(\underline{\eta}_{r}^{\prime}, \underline{\mu}_{r}, \underline{\rho}_{r}\right)^{-1}$, and for brevity we have written $(\cdot)$ for $\left(\eta_{r, \ell}^{\prime}, \zeta_{r, \ell}, \mu_{r, \ell}, \rho_{r, \ell}\right)$.

Referring again to the texts preceding (3.15) and (3.23), respectively, for notation, let $S_{r}\left(\epsilon_{r}\right)=\left\{\widetilde{\eta}^{\prime} \in \mathbb{R}^{n-1} \mid\left(\widetilde{\eta}_{r}, \widetilde{\mu}\right) \in \widetilde{\Sigma}_{r}\right.$ for $\left.\widetilde{\mu} \in \mathcal{L}_{1}\right\}$ and let $\left(\widetilde{\eta}_{0}^{\prime}, \mu_{0}\right)$ denote an arbitrary point of $S_{r}\left(\epsilon_{r}\right) \times \mathcal{L}_{1}$. Then it follows from Definition 2.6 that 
there is a neighbourhood of $\left(\widetilde{\eta}_{0}^{\prime}, \mu_{0}\right)$ in $\mathbb{R}^{n-1} \times \mathbb{C}$, say $U\left(\widetilde{\eta}_{0}^{\prime}, \mu_{0}\right)$, such that for $\left(\widetilde{\eta}^{\prime}, \mu\right) \in U\left(\widetilde{\eta}_{0}^{\prime}, \mu_{0}\right) \cap\left(S_{r}\left(\epsilon_{r}\right) \times \mathcal{L}_{1}\right),\left(\widetilde{\eta}^{\prime}, \widetilde{\mu}\right) \in \Sigma_{r}$ and all assertions concerning (3.15)(3.18) hold with $\widetilde{\mu}=\left(1-\left|\widetilde{\eta}^{\prime}\right|^{2}\right)^{\tilde{m}_{r} / 2} \mu, \xi^{\prime}=\rho_{r, r} \widetilde{\eta}^{\prime}, z=\rho_{r, r} \zeta$ and $\mu=\rho_{r, r}^{\tilde{m}_{r}} \widetilde{\mu}$, while for $r<\ell \leq d$, all assertions concerning (3.23)-(3.26) hold with $\widetilde{\mu}=\mu, z=\rho_{r, \ell} \zeta$, and $\lambda=\rho_{r, \ell} \mu$. Since $S_{r}\left(\epsilon_{r}\right) \times \mathcal{L}_{1}$ is compact, it is covered by a finite number of such opens sets, say $\left\{U_{j}\right\}_{j=1}^{M}$, and we let $\left\{\phi_{j}\left(\widetilde{\eta}^{\prime}, \mu\right)\right\}_{j=1}^{M}$ denote a partition of unity subordinate to this covering of $S_{r}\left(\epsilon_{r}\right) \times \mathcal{L}_{1}$.

Let $\widetilde{S}_{r}\left(\epsilon_{r}\right)=\left\{\widetilde{\eta}^{\prime} \in S_{r}\left(\epsilon_{r}\right)|| \widetilde{\eta}^{\prime} \mid<1\right\}$ and $\Sigma\left(\lambda^{0}\right)=\left\{\left(\xi^{\prime}, \lambda\right) \in \mathbb{R}^{n-1} \times \mathcal{L}|| \lambda \mid \geq\right.$ $\left.\lambda^{0}, \frac{1}{8} \epsilon_{r}|\lambda|^{1 / \widetilde{m}_{r}} \leq\left|\xi^{\prime}\right| \leq \frac{7}{8} \epsilon_{r+1}|\lambda|^{1 / \widetilde{m}_{r+1}}\right\}$. Then we observe that each pair $\left(\xi^{\prime}, \lambda\right) \in$ $\Sigma\left(\lambda^{0}\right)$ uniquely determines the point $\left(\widetilde{\eta}_{r, r}^{\prime}, \mu\right) \in U_{j} \cap\left(\widetilde{S}_{r}\left(\epsilon_{r}\right) \cap \mathcal{L}_{1}\right)$ for some $j$, where $\widetilde{\eta}_{r, r}^{\prime}=\rho_{r, r}^{-1} \xi^{\prime}$,

$$
\left|\widetilde{\eta}_{r, r}^{\prime}\right| \leq \frac{\frac{7}{8} \epsilon_{r+1}}{\left(\left(\frac{7}{8} \epsilon_{r+1}\right)^{2}+|\lambda|^{\left.2 / \widetilde{m}_{r}-2 / \widetilde{m}_{r+1}\right)^{1 / 2}}\right.}
$$

$\mu=\frac{\lambda}{|\lambda|}$, and $\rho_{r, r}$ is defined as before. Conversely, the set of all such pairs $\left(\xi^{\prime}, \lambda\right)$ is contained in $\bigcup_{j=1}^{M} \widetilde{U}_{j}$, where

$$
\begin{aligned}
& \widetilde{U}_{j}=\left\{\left(\xi^{\prime}, \lambda\right) \in \mathbb{R}^{n-1} \times \mathcal{L}\left|\xi^{\prime}=\tau \widetilde{\eta}^{\prime},\right| \lambda \mid=\tau^{\widetilde{m}_{r}}\left(1-\left|\widetilde{\eta}^{\prime}\right|^{2}\right)^{\widetilde{m}_{r} / 2} \mu\right. \\
& \left.\quad \text { for } \tau \geq\left(\frac{\lambda^{0}}{\left(1-\left|\widetilde{\eta}^{\prime}\right|^{2}\right)^{\tilde{m}_{r} / 2}}\right)^{1 / \widetilde{m}_{r}}, \text { and }\left(\widetilde{\eta}^{\prime}, \mu\right) \in U_{j} \cap\left(\widetilde{S}_{r}\left(\epsilon_{r}\right) \times \mathcal{L}_{1}\right)\right\} .
\end{aligned}
$$

An immediate consequence of these considerations is that if we henceforth agree to replace $\mathcal{G}^{(r, \ell)}(\cdot)$ in $(3.40)$ by $\mathcal{G}_{j}^{(r, \ell)}(\cdot)$ whenever $\left(\xi^{\prime}, \lambda\right) \in \Sigma\left(\lambda^{0}\right) \cap \widetilde{U}_{j}$, and let $G^{(r, \ell)}(\cdot)=\sum_{j=1}^{M} \phi_{j}\left(\eta_{r, r}^{\prime}, \mu_{r, r+1}\right) \mathcal{G}_{j}^{(r, \ell)}(\cdot) \chi_{\lambda}^{(r)}\left(\xi^{\prime}\right)$, where for brevity we have written (.) for $\left(\underline{\eta}_{r}^{\prime}, \zeta_{r, \ell}, \underline{\mu}_{r}, \underline{\rho}_{r}\right)$, then we obtain a solution

$$
\begin{aligned}
u^{(r)}\left(\xi^{\prime}, x_{n}, \lambda\right)=\sum_{\ell=r}^{d} & \operatorname{diag}\left(\rho_{r, \ell}^{-t_{1}}, \ldots, \rho_{r, \ell}^{-t_{N}}\right)\left(\int_{\gamma_{r, \ell}^{+}} e^{i \rho_{r, \ell} x_{n} \zeta_{r, \ell}} G^{(r, \ell)}\left(\underline{\eta}_{r}^{\prime}, \zeta_{r, \ell} \underline{\mu}_{r}, \underline{\rho}_{r}\right) d \zeta_{r, \ell}\right) \\
& \times \operatorname{diag}\left(\widetilde{\rho}_{1}^{-\sigma_{1}}, \ldots, \widetilde{\rho}_{N_{d}}^{-\sigma_{N_{d}}}\right) g^{(r)}\left(\xi^{\prime}\right)
\end{aligned}
$$

of (3.8), (3.39) for all values of $\xi^{\prime} \in \mathbb{R}^{n-1}$ and $\lambda \in \mathcal{L}_{1}$ with $|\lambda| \geq \lambda^{0}$ with the property that each entry of

$$
G^{(r, \ell)}\left(\underline{\eta}_{r}^{\prime}, \zeta_{r, \ell}, \underline{\mu}_{r}, \underline{\rho}_{r}\right)=\left(G_{j k}^{(r, \ell)}\left(\underline{\eta}_{r}^{\prime}, \zeta_{r, \ell}, \underline{\mu}_{r}, \underline{\rho}_{r}\right)\right)_{\substack{j=1, \ldots, N \\ k=1, \ldots, N_{d}}}
$$

has derivatives of all orders with respect to the components of $\xi^{\prime}$ which are continuous in $\xi^{\prime}$ and $\lambda$, and, in addition, for any multi-index $\alpha^{\prime}$ whose entries are either 0 or 1 ,

$$
\left|\xi^{\prime \alpha^{\prime}} D_{\xi^{\prime}}^{\prime \alpha^{\prime}} G_{j, k}\left(\underline{\eta}_{r}^{\prime}, \zeta_{r, \ell}, \underline{\mu}_{r}, \underline{\rho}_{r}\right)\right| \leq C \quad\left(\text { resp. }|\lambda|^{-1} C\right) \quad \text { if } j \leq k_{\ell} \quad\left(\text { resp. } j>k_{\ell}\right),
$$

where the constant $C$ does not depend upon $\xi^{\prime}, \lambda$, and $\alpha^{\prime}$.

In light of (3.41) we see that the proposition will be proved for the case under consideration here if we can show that $\left\|u_{j, k, \ell}^{(r)}\right\|_{t_{j}, p, \mathbb{R}_{+}^{n}}^{\left(\pi_{1}(j)\right)}$ is bounded by the expression on the right-hand side of (3.38) for every $j, k$, and $\ell$ satisfying $1 \leq j \leq N, 1 \leq k \leq$ $N_{d}$, and $r \leq \ell \leq d$ with $\pi_{2}(k)=\ell$, where

$$
u_{j, k, \ell}^{(r)}\left(\xi^{\prime}, x_{n}, \lambda\right)=\rho_{r, \ell}^{-t_{j}}\left(\int_{\gamma_{r, \ell}^{+}} e^{i \rho_{r, \ell} x_{n} \zeta_{r, \ell}} G_{j, k}^{(r, \ell)}\left(\underline{\eta}_{r}^{\prime}, \zeta_{r, \ell}, \underline{\mu}_{r}, \underline{\rho}_{r}\right) d \zeta_{r, \ell}\right) \rho_{r, \ell}^{-\sigma_{k}} g_{k}^{(r)}\left(\xi^{\prime}\right) \text {. }
$$


To this end let us observe that

$$
\begin{aligned}
\left\|u_{j, k, \ell}^{(r)}\right\|_{t_{j}, p, \mathbb{R}_{+}^{n}}^{\left(\pi_{1}(j)\right)} \leq & C \sum_{m=0}^{t_{j}} \| F^{\prime-1}\left\langle\xi^{\prime}, \lambda\right\rangle_{\pi_{1}(j)}^{m} \rho^{-\sigma_{k}-m} \\
& \times\left(\int_{\gamma^{+}} e^{i x_{n} \rho \zeta} \zeta^{t_{j}-m} G_{j, k}^{(r, \ell)}\left(\underline{\eta}_{r}^{\prime}, \zeta, \underline{\mu}_{r}, \underline{\rho}_{r}\right) d \zeta\right) F^{\prime} g_{k}^{(r)}\left(\xi^{\prime}\right) \|_{0, p, \mathbb{R}_{+}^{n}}
\end{aligned}
$$

where for brevity we have put $\rho=\rho_{r, \ell}, \zeta=\zeta_{r, \ell}, \gamma^{+}=\gamma_{r, \ell}^{+}$, and where $C$ denotes a constant not depending upon $g^{(r)}$ and $\lambda$. Hence if we put $\Lambda\left(\xi^{\prime}, \lambda\right)=\left\langle\xi^{\prime}\right\rangle$ if $\ell=r$, $\Lambda\left(\xi^{\prime}, \lambda\right)=\left\langle\xi^{\prime}, \lambda\right\rangle_{\ell}$ if $\ell>r$, and $h(x, \lambda)=\left(F^{\prime-1} e^{-\Lambda\left(\xi^{\prime}, \lambda\right) x_{n}} F^{\prime} g_{k}^{(r)}\right)(x)$ for $x \in \mathbb{R}_{+}^{n}$ (see [ADF, Proposition 2.3]), argue as in [V, Section 5], and appeal to Minkowski's inequality, then we deduce that

$$
\left\|u_{j, k, \ell}^{(r)}\right\|_{t_{j}, p, \mathbb{R}_{+}^{n}}^{\left(\pi_{1}(j)\right)} \leq C^{\prime} \sum_{\nu=1}^{2}\left[\int_{x_{n}=0}^{\infty} d x_{n}\left(\int_{\tau=0}^{\infty} J_{\nu}\left(x_{n}, \tau, \lambda\right) d \tau\right)^{p}\right]^{1 / p}
$$

where

$$
\begin{aligned}
& J_{1}\left(x_{n}, \tau, \lambda\right)=\left(x_{n}+\tau\right)^{-1}\left\|F^{\prime-1} \rho^{-\sigma_{k}} F^{\prime} h\left(s^{\prime}, \tau\right) \widetilde{J}_{1}\left(x_{n}, \tau, \xi^{\prime}, \lambda\right)\right\|_{0, p, \mathbb{R}^{n-1}}, \\
& J_{2}\left(x_{n}, \tau, \lambda\right)=\left(x_{n}+\tau\right)^{-1}\left\|F^{\prime-1} \rho^{-\sigma_{k}-1} F^{\prime} D_{\tau} h\left(s^{\prime}, \tau\right) \widetilde{J}_{2}\left(x_{n}, \tau, \xi^{\prime}, \lambda\right)\right\|_{0, p, \mathbb{R}^{n-1},},{ }^{\prime}{ }^{\prime}\left(x_{n}, \tau, \xi^{\prime}, \lambda\right) \\
& =\int_{\gamma^{+}} \rho \zeta\left(x_{n}+\tau\right) e^{i \rho \zeta\left(x_{n}+\tau\right)}\left\langle\xi^{\prime}, \lambda\right\rangle_{\pi_{1}(j)}^{m} \rho^{-m} G_{j k}^{(r, \ell)}\left(\underline{\eta}_{r}^{\prime}, \zeta, \underline{\mu}_{r}, \underline{\rho}_{r}\right) \zeta^{t_{j}-m} d \zeta, \\
& \widetilde{J}_{2}\left(x_{n}, \tau, \xi^{\prime}, \lambda\right) \\
& =\int_{\gamma^{+}} \rho \zeta\left(x_{n}+\tau\right) e^{i \rho \zeta\left(x_{n}+\tau\right)}\left\langle\xi^{\prime}, \lambda\right\rangle_{\pi_{1}(j)}^{m} \rho^{-m} G_{j k}^{(r, \ell)}\left(\underline{\eta}_{r}^{\prime}, \zeta, \underline{\mu}_{r}, \underline{\rho}_{r}\right) \zeta^{t_{j}-1-m} d \zeta,
\end{aligned}
$$

and the constant $C^{\prime}$ does not depend upon $g^{(r)}$ and $\lambda$.

Let us firstly fix our attention upon the case where $\pi_{2}(k) \leq r$, so that now we have $\ell=r$ in (3.42)-(3.44). Then with the use of the Mikhlin-Lizorkin multiplier theorem we can show that for $1 \leq \nu \leq 2$,

$$
\left(\frac{\rho}{\left\langle\xi^{\prime}\right\rangle}\right)^{-\sigma_{k}-\nu+1} \widetilde{J}_{\nu}\left(x_{n}, \tau, \xi^{\prime}, \lambda\right) \chi_{\lambda}^{(r)}\left(\xi^{\prime}\right)
$$

is a Fourier multiplier of type $(p, p)$ with norm bounded by a constant not depending upon $g^{(r)}, x_{n}, \tau$, and $\lambda$. Hence it follows from (3.42)-(3.44) and [ADF, Proposition 2.3] that

$$
\left\|u_{j, k, \ell}^{(r)}\right\|_{t_{j}, p, \mathbb{R}_{+}^{n}}^{\pi_{1}(j)} \leq C_{r}\left\|g_{k}^{(r)}\right\|_{-\sigma_{k}-\frac{1}{p}, p, \mathbb{R}^{n-1}} \leq C_{r}\left\|g_{k}^{(r)}\right\|_{-\sigma_{k}-\frac{1}{p}, p, \mathbb{R}^{n-1}}^{\pi_{2}(k)},
$$

where the constant $C_{r}$ does not depend upon $g^{(r)}$ and $\lambda$.

Turning next to the case where $\pi_{2}(k)=\ell>r$, we can again show that for $1 \leq \nu \leq 2$

$$
\left(\frac{\rho}{\left\langle\xi^{\prime}, \lambda\right\rangle_{\pi_{2}(k)}}\right)^{-\sigma_{k}-\nu+1} \widetilde{J}_{\nu}\left(x_{n}, \tau, \xi^{\prime}, \lambda\right) \chi_{\lambda}^{(r)}\left(\xi^{\prime}\right)
$$

is a Fourier multiplier of type $(p, p)$ with norm bounded by a constant not depending upon $g^{(r)}, x_{n}, \tau$, and $\lambda$. Thus it follows from (3.42)-(3.44) and [ADF, Proposition 
2.3] that

$$
\left\|u_{j, k, \ell}^{(r)}\right\|_{t_{j}, p, \mathbb{R}_{+}^{n}}^{\pi_{1}(j)} \leq C_{\ell}\left\|g_{k}^{(r)}\right\|_{-\sigma_{k}-\frac{1}{p}, p, \mathbb{R}^{n-1}}^{\pi_{2}(k)},
$$

and the constant $C_{\ell}$ has the same properties as the constant $C$ above; and this completes the proof of the proposition.

Proof of Proposition 3.6. In proving the proposition there is no loss of generality in supposing that $g \in \mathcal{S}\left(\mathbb{R}^{n-1}\right)^{\tilde{N}}$ since the result for $g \in \prod_{j=1}^{\tilde{N}} W_{p}^{-\sigma_{j}-\frac{1}{p}}\left(\mathbb{R}^{n-1}\right)$ will then follow from a standard approximation procedure. Accordingly, under a Fourier transformation with respect to $x^{\prime}\left(x^{\prime} \mapsto \xi^{\prime}\right)$, the boundary problem $(3.3),(3.4)$ is transformed into the boundary problem (3.8), (3.39). Then assuming that $\epsilon^{0}$ and $\lambda^{0}$ have been chosen so that the conclusions of Propositions 3.12 and 3.14 hold, it follows from [DV, Theorem 3.5] and the above considerations that for a fixed $\lambda \in \mathcal{L}$ with $|\lambda| \geq \lambda_{0}$ this boundary problem has a unique solution $u\left(\xi^{\prime}, x_{n}, \lambda\right)$ in the space of vector functions decaying exponentially at $\infty$, and hence $u\left(\xi^{\prime}, x_{n}, \lambda\right)=$ $\sum_{r=0}^{d} u^{(r)}\left(\xi^{\prime}, x_{n}, \lambda\right)$. The assertions of the proposition follow directly from the results of Proposition 3.15 and the Mikhlin-Lizorkin multiplier theorem.

\section{Proof of Theorem 2.8}

We suppose henceforth that the hypotheses of Theorem 2.8 hold. Moreover, by employing a standard extension procedure we can also assume that the $a_{\alpha}^{j k}(x)$ and $b_{\alpha}^{j k}(x)$ of $(2.1)$ are defined on all of $\mathbb{R}^{n}$, are compactly supported, and satisfy the same smoothness assumptions as asserted in Assumption 2.2 for $\bar{\Omega}$ and $\Gamma$, respectively. Lastly for $x^{0} \in \bar{\Omega}$ and $\delta>0$, let $B_{\delta}\left(x^{0}\right)$ denote the open ball in $\mathbb{R}^{n}$ with centre $x^{0}$ and radius $\delta$. Then in order to prove the theorem we require some further results.

Proposition 4.1. For any $\epsilon>0$ and $x^{0} \in \bar{\Omega}$ there exists a $\delta>0$ and $a \lambda^{0}>0$ such that for $\lambda \in \mathcal{L}$ with $|\lambda| \geq \lambda^{0}$

$$
\sum_{j=1}^{N}\|\| \sum_{k=1}^{N}\left(A_{j k}(x, D)-\AA_{j k}\left(x^{0}, D\right)\right) u_{k}\|\|_{-s_{j}, p, \Omega}^{\left(\pi_{1}(j)\right)} \leq \epsilon \sum_{j=1}^{N}\left\|u_{j}\right\|_{t_{j}, p, \Omega}
$$

for every $u \in \prod_{j=1}^{N} W_{p}^{t_{j}}(\Omega)$ such that supp $u \subset B_{\delta}\left(x^{0}\right) \cap \bar{\Omega}$.

Proof. Let us fix a $j, 1 \leq j \leq N$, and suppose firstly that $s_{j}>0$. Then we have two cases to consider: (1) $x^{0} \in \Omega$ and $(2) x^{0} \in \Gamma$.

Let $x^{0} \in \Omega$ and suppose that $B_{\delta}\left(x^{0}\right) \subset \Omega$. Then for $1 \leq k \leq N$ we have

$$
\begin{aligned}
\left(A_{j k}(x, D)-\AA_{j k}\left(x^{0}, D\right)\right) u_{k} & =\sum_{|\alpha|=s_{j}+t_{k}}\left(a_{\alpha}^{j k}(x)-a_{\alpha}^{j k}\left(x^{0}\right)\right) D^{\alpha} u_{k} \\
& +\sum_{|\alpha|<s_{j}+t_{k}} a_{\alpha}^{j k}(x) D^{\alpha} u_{k} .
\end{aligned}
$$

It is clear that for a fixed $\alpha,\left(a_{\alpha}^{j k}(x)-a_{\alpha}^{j k}\left(x^{0}\right)\right) D^{\alpha} u_{k}$ (resp. $\left.a_{\alpha}^{j k}(x) D^{\alpha} u_{k}\right)$ is a distribution on $\mathbb{R}^{n}$ of order $s_{j}$ (resp. $\leq s_{j}$ ) whose support is contained in that of $u_{k}$. Hence it follows from [GK, Section 1], [T, Theorem 2.6.1, p.198] that for 


$$
\begin{aligned}
& |\alpha|=s_{j}+t_{k}, \\
& \qquad \begin{aligned}
\left\|\left(a_{\alpha}^{j k}(x)-a_{\alpha}^{j k}\left(x^{0}\right)\right) D^{\alpha} u_{k}\right\|_{-s_{j}, p, \Omega}^{\left(\pi_{1}(j)\right)} & \leq\left\|a_{\alpha}^{j k}(x)-a_{\alpha}^{j k}\left(x^{0}\right) D^{\alpha} u_{k}\right\|_{-s_{j}, p, \mathbb{R}^{n}}^{\left(\pi_{1}(j)\right)} \\
& =\sup _{\zeta}\left|\left\langle\left(a_{\alpha}^{j k}(x)-a_{\alpha}^{j k}\left(x^{0}\right)\right) D^{\alpha} u_{k}, \zeta\right\rangle\right| \\
& =\sup _{\zeta}\left|\left\langle D^{\alpha-\beta} u_{k}, D^{\beta}\left(a_{\alpha}^{j k}(x)-a_{\alpha}^{j k}\left(x^{0}\right)\right) \zeta\right\rangle\right|,
\end{aligned}
\end{aligned}
$$

where $\langle\cdot, \cdot\rangle$ denotes the pairing between $H_{p}^{-s_{j}}\left(\mathbb{R}^{n}\right)$ and its dual, the supremum is taken over all $\zeta \in C_{0}^{\infty}\left(\mathbb{R}^{n}\right)$ satisfying $\|\zeta\|_{s_{j}, p^{\prime}, \mathbb{R}^{n}}^{\left(\pi_{1}(j)\right.} \leq 1$, and $|\beta|=s_{j}$. Note that here duality between the spaces indicated is to be understood in the sense that the spaces are now equipped not with their standard norms, but with equivalent norms, namely their parameter dependent norms $\|\cdot\|_{-s_{j}, p, \mathbb{R}^{n}}^{\left(\pi_{1}(j)\right)}$ and $\|\cdot\|_{s_{j}, p^{\prime}, \mathbb{R}^{n}}^{\left(\pi_{1}(j)\right)}$, respectively (see [GK, Eqn. (1.9)]). We conclude from (4.3) that the expression on the left side of this equation does not exceed $\left\|u_{k}\right\|_{t_{k}, p, \mathbb{R}^{n}}\left(\rho\|\zeta\|_{s_{j}, p^{\prime}, \mathbb{R}^{n}}+C\|\zeta\|_{s_{j}-1, p^{\prime}, \mathbb{R}^{n}}\right)$, where $\rho=\sup _{x \in B_{\delta}\left(x^{0}\right)}\left|a_{\alpha}^{j k}(x)-a_{\alpha}^{j k}\left(x^{0}\right)\right|$, and here and below $C$ denotes a generic constant that does not depend upon $u_{k}, \lambda$, and $\zeta$. Thus it follows that the expression on the left side of (4.3) does not exceed $\left(C|\lambda|^{-\frac{1}{\bar{m}_{\pi_{1}(j)}}}+\rho\right)\left\|u_{k}\right\|_{t_{k}, p, \mathbb{R}^{n}}$. On the other hand if $|\alpha|<s_{j}+t_{k}$, then similar arguments show that $\left\|a_{\alpha}^{j k}(x) D^{\alpha} u_{k}\right\|_{-s_{j}, p, \Omega}^{\left(\pi_{1}(j)\right)} \leq$ $C|\lambda|^{-\frac{1}{\bar{m}_{\pi_{1}(j)}}}\left\|u_{k}\right\|_{t_{k}, p, \mathbb{R}^{n}}$.

Suppose next that $x^{0} \in \Gamma$ and let $\{U, \phi\}$ be a chart on $\Gamma$ such that $x^{0} \in$ $U, \phi\left(x^{0}\right)=0$, and $\phi$ is a diffeomorphism of class $C^{\kappa_{0}-1,1} \cap C^{s_{1}}$ mapping $U$ onto an open set in $\mathbb{R}^{n}$ with $\phi(U \cap \Omega) \subset \mathbb{R}_{+}^{n}$ and $\phi(U \cap \Gamma) \subset \mathbb{R}^{n-1}$. We suppose henceforth that $B_{2 \delta}\left(x^{0}\right) \subset U$. Then for $1 \leq k \leq N$ we have the representation (4.2), from which it follows that for a fixed $\alpha,\left(a_{\alpha}^{j k}(x)-a_{\alpha}^{j k}\left(x^{0}\right)\right) D^{\alpha} u_{k}\left(\right.$ resp. $\left.a_{\alpha}^{j k}(x) D^{\alpha} u_{k}\right)$ is a distribution on $\Omega$ of order $s_{j} \quad\left(\right.$ resp. $\leq s_{j}$ ) whose support is contained in that of $u_{k}$. Furthermore, it is clear from the definition that $\|v\|_{-s_{j}, p, \Omega}^{\left(\pi_{1}(j)\right)} \geq\|v\|_{-s_{j}, p, \Omega \cap U}^{\left(\pi_{1}(j)\right)}$ for both (1) $v=\left(a_{\alpha}^{j k}(x)-a_{\alpha}^{j k}\left(x^{0}\right)\right) D^{\alpha} u_{k}$ and (2) $v=a_{\alpha}^{j k}(x) D^{\alpha} u_{k}$. To obtain a reverse estimate, let us firstly fix our attention upon the $v$ of case (1), choose $w \in H_{p}^{-s_{j}}\left(\mathbb{R}^{n}\right)$ such that $\left.w\right|_{\Omega \cap U}=v$ and $\|w\|_{-s_{j}, p, \mathbb{R}^{n}}^{\left(\pi_{1}(j)\right)} \leq 2\|v\|_{-s_{j}, p, \Omega \cap U}^{\left(\pi_{1}(j)\right)}$, and let $\psi \in C_{0}^{\infty}\left(\mathbb{R}^{n}\right)$ such that $\psi(x)=1$ for $x \in B_{\delta}\left(x^{0}\right)$ and $\operatorname{supp} \psi \subset U$. The $\psi w \in H_{p}^{-s_{j}}\left(\mathbb{R}^{n}\right),\left.\psi w\right|_{\Omega}=v$, and hence

$$
\|v\|_{-s_{j}, p, \Omega}^{\left(\pi_{1}(j)\right)} \leq\|\psi w\|_{-s_{j}, p, \mathbb{R}^{n}}^{\left(\pi_{1}(j)\right)} \leq C\|w\|_{-s_{j}, p, \mathbb{R}^{n}}^{\left(\pi_{1}(j)\right)} \leq 2 C\|v\|_{-s_{j}, p, \Omega \cap U}^{\left(\pi_{1}(j)\right)},
$$

where we have used an argument similar to that used in (4.3). Likewise we can show that $\left\|a_{\alpha}^{j k}(x) D^{\alpha} u_{k}\right\|_{-s_{j}, p, \Omega}^{\left(\pi_{1}(j)\right)} \leq C\left\|a_{\alpha}^{j k}(x) D^{\alpha} u_{k}\right\|_{-s_{j}, p, \Omega \cap U}^{\left(\pi_{1}(j)\right)}$.

By means of the mapping $\phi$ we can now pass to the local coordinates at $x^{0}$, and in these local coordinates $\left(a_{\alpha}^{j k}(x)-a_{\alpha}^{j k}\left(x^{0}\right)\right) D^{\alpha} u_{k}$ can be written as

$$
\phi_{*}\left(\left(a_{\alpha}^{j k}(x)-a_{\alpha}^{j k}\left(x^{0}\right)\right) D^{\alpha} u_{k}\right)=\left(\widetilde{a}_{\alpha}^{j k}(y)-\widetilde{a}_{\alpha}^{j k}(0)\right) D_{y}^{\alpha} \widetilde{u}_{k}+\sum_{0<|\beta|<|\alpha|} \widetilde{a}_{\alpha}^{j k}(y) D_{y}^{\beta} \widetilde{u}_{k},
$$

where $y=\phi(x)$. Furthermore, from the results given in [GK, Section 1], [T, Theorem 4.8.1, p.332] and the proofs of Lemma 4.2 and Theorems 4.7.2 and 4.8.2 
of [T, pp.310-311 and 332-333] it is not difficult to deduce that

$$
\begin{aligned}
& \left\|\left(a_{\alpha}^{j k}(x)-a_{\alpha}^{j k}\left(x^{0}\right)\right) D^{\alpha} u_{k}\right\|_{-s_{j}, p, \Omega \cap U}^{\left(\pi_{1}(j)\right)} \\
& \leq C\left\|\phi_{*}\left(\left(a_{\alpha}^{j k}(x)-a_{\alpha}^{h j k}\left(x^{0}\right)\right) D^{\alpha} u_{k}\right)\right\|_{-s_{j}, p, \phi(\Omega \cap U)}^{\left(\pi_{1}(j)\right)} \\
& \leq C\left\|\phi_{*}\left(\left(a_{\alpha}^{j k}(x)-a_{\alpha}^{j k}\left(x^{0}\right)\right) D^{\alpha} u_{k}\right)\right\|_{-s_{j}, p, \mathbb{R}_{+}^{n}}^{\left(\pi_{1}(j)\right)} \\
& \leq C\left(\sup _{\zeta}\left|\left\langle D_{y}^{\alpha} \widetilde{u}_{k},\left(\widetilde{a}_{\alpha}^{j k}(y)-\widetilde{a}_{\alpha}^{j k}(0)\right) \widetilde{\psi} \zeta\right\rangle\right|+\sum_{0<|\beta|<|\alpha|} \sup _{\zeta}\left|\left\langle D_{y}^{\beta} \widetilde{u}_{k}, \widetilde{a}_{\beta}^{j k}(y) \widetilde{\psi} \zeta\right\rangle\right|\right),
\end{aligned}
$$

where the constant $C$ does not depend upon $u_{k},\langle\cdot, \cdot\rangle$ denotes the pairing between $H_{p}^{-s_{j}}\left(\mathbb{R}_{+}^{n}\right)$ and its dual $\stackrel{\circ}{W}_{p^{\prime}}^{s_{j}}\left(\mathbb{R}_{+}^{n}\right)$, equipped with their parameter dependent norms $\|\cdot\|_{-s_{j}, p, \mathbb{R}_{+}^{n}}^{\left(\pi_{1}(j)\right)}$ and $\|\cdot\|_{s_{j}, p^{\prime}, \mathbb{R}_{+}^{n}}^{\left(\pi_{1}(j)\right)}$, respectively, the supremum is taken over the set $\left\{\zeta \in C_{0}^{\infty}\left(\mathbb{R}_{+}^{n}\right) \mid\|\zeta\|_{s_{j}, p^{\prime}, \mathbb{R}_{+}^{n}}^{\left(\pi_{1}(j)\right.} \leq 1\right\}, \widetilde{\psi}=\phi_{*}(\psi)$, and $\psi \in C_{0}^{\infty}\left(\mathbb{R}^{n}\right)$ such that $\psi(x)=1$ for $x \in B_{\delta}\left(x^{0}\right), \operatorname{supp} \psi \subset B_{2 \delta}\left(x^{0}\right)$. From this last result it is not difficult to deduce that the expression on the left side of (4.4) does not exceed $C\left(\rho+C_{1}|\lambda|^{-\frac{1}{m_{\pi_{1}(j)}}}\right)\left\|u_{k}\right\|_{t_{k}, p, \Omega \cap U}$, where $\rho=\sup _{x \in B_{2 \delta}\left(x^{0}\right)}\left|\left(a_{\alpha}^{j k}(x)-a_{\alpha}^{j k}\left(x^{0}\right)\right)\right|$ and the constant $C_{1}$ does not depend upon $u_{k}$ and $\lambda$. Since a similar result holds for $\left\|a_{\alpha}^{j k}(x) D^{\alpha} u_{k}\right\|_{-s_{j}, p, \Omega \cap U}^{\left(\pi_{1}(j)\right)}$ for $|\alpha|<s_{j}+t_{k}$, the proof of the proposition is complete for the case $s_{j}>0$; the proof for the case $s_{j}=0$ follows from a standard argument.

Arguments similar to those used in the proof of Proposition 4.1 can also be used to prove the following two results.

Proposition 4.2. For any $\epsilon>0$ and $x^{0} \in \Omega$ there exits a $\delta, 0<\delta<\operatorname{dist}\left\{x^{0}, \Gamma\right\}$, and $a \lambda^{0}>0$ such that if $\operatorname{supp}\left(a_{\alpha}^{j k}(x)-a_{\alpha}^{j k}\left(x^{0}\right)\right) \subset B_{\delta}\left(x^{0}\right)$ for $|\alpha|=s_{j}+t_{k}, j, k=$ $1, \ldots, N$, and $\lambda \in \mathcal{L}$ with $|\lambda| \geq \lambda^{0}$, then the estimate

$$
\sum_{j=1}^{N} \sum_{k=1}^{N}\left\|\left(\AA_{j k}(x, D)-\AA_{j k}\left(x^{0}, D\right)\right) u_{k}\right\|_{-s_{j}, p, \mathbb{R}^{n}}^{\left(\pi_{1}(j)\right)} \leq \epsilon \sum_{j=1}^{N}\left\|u_{k}\right\|_{t_{k}, p, \mathbb{R}^{n}}
$$

holds for every $u \in \prod_{j=1}^{N} W_{p}^{t_{j}}\left(\mathbb{R}^{n}\right)$.

We wish now to consider the analogue of Proposition 4.2 for the case $x^{0} \in$ $\Gamma$. Accordingly, let us turn again to the chart $\{U, \phi\}$ introduced in the proof of Proposition 4.1. Then by means of the mapping $\phi$ we can now pass to local coordinates at $x^{0}$, and in the local coordinates each of the operators $A_{j k}(x, D)$ can be written as $\widetilde{A}_{j k}(y, D)=\sum_{|\alpha| \leq s_{j}+t_{k}} \widetilde{a}_{\alpha}^{j k}(y) D_{y}^{\alpha}$ for $y \in \phi(U)$. We are interested in the case where for some ball $\overline{B_{\delta}(0)} \subset \phi(U), \widetilde{a}_{\alpha}^{j k}(y)=\widetilde{a}_{\alpha}^{j k}(0)$ for $y \in \phi(U) \backslash B_{\delta}(0)$ and for all $\alpha$ satisfying $|\alpha|=s_{j}+t_{k}$. Because of this assumption we can now extend the operator $\stackrel{o}{A}_{j k}\left(y, D_{y}\right)$, the principal part of $\widetilde{A}_{j k}\left(y, D_{y}\right)$, to an operator on all of $\mathbb{R}^{n}$ by putting $\widetilde{a}_{\alpha}^{j k}(y)=\widetilde{a}_{\alpha}^{j k}(0)$ for $y \in \mathbb{R}^{n} \backslash \phi(U)$ and $|\alpha|=s_{j}+t_{k}$.

Proposition 4.3. For any $\epsilon>0$ there exists a $\delta, 0<\delta<\operatorname{dist}\{0, \partial \phi(U)\}$, and $a$ $\lambda^{0}>0$ such that if $\operatorname{supp}\left(\widetilde{a}_{\alpha}^{j k}(y)-\widetilde{a}_{\alpha}^{j k}(0)\right) \subset B_{\delta}(0)$ for $|\alpha|=s_{j}+t_{k}, j, k=1, \ldots, N$ 
and $\lambda \in \mathcal{L}$ with $|\lambda| \geq \lambda^{0}$, then the estimate

$$
\sum_{j=1}^{N} \sum_{k=1}^{N}\left\|\left(\stackrel{o}{\widetilde{A}}\left(y, D_{y}\right)-\stackrel{o}{\widetilde{A}}\left(0, D_{y}\right)\right) u_{k}\right\|_{-s_{j}, p, \mathbb{R}_{+}^{n}}^{\left(\pi_{1}(j)\right)} \leq \epsilon \sum_{j=1}^{N}\left\|u_{k}\right\|_{t_{k}, p, \mathbb{R}_{+}^{n}}
$$

holds for every $u \in \prod_{j=1}^{N} W_{p}^{t_{j}}\left(\mathbb{R}_{+}^{n}\right)$.

Finally, the proof of the theorem follows directly from the results of Section 3, Propositions 4.1 - 4.3, and the arguments used in the proofs of Theorems 4.1 and 5.1 of $[\mathrm{AV}]$.

\section{REFERENCES}

[ADN] S. Agmon, R. Douglis, and L. Nirenberg: Estimates near the boundary for solutions of elliptic partial differential equations satisfying general boundary conditions II. Comm. Pure Appl. Math. 17 (1964), 35-92.

[ADF] M.S. Agranovich, R. Denk, and M. Faierman: Weakly smooth nonselfadjoint elliptic boundary problems. In: Advances in partial differential equations: Spectral theory, microlocal analysis, singular manifolds, Math. Top. 14 (1997), 138-199.

[AV] M.S. Agranovich and M.I. Vishik: Elliptic problems with a parameter and parabolic problems of general form. Russ. Math. Surveys 19 (1964), 53-157.

[DFM] R. Denk, M. Faierman, and M. Möller: An elliptic boundary problem for a system involving a discontinuous weight. Manuscripta Math. 108 (2001), 289-317.

[DMV] R. Denk, R. Mennicken, and L. Volevich: The Newton polygon and elliptic problems with parameter. Math. Nachr. 192 (1998), 125-157.

[DV] R. Denk and L. Volevich: Elliptic boundary value problems with large parameter for mixed order systems. Amer. Math. Soc. Transl.(2) 206 (2002), 29-64.

[F] M. Faierman: Eigenvalue asymptotics for a boundary problem involving an elliptic system. Math. Nachr. 279 (2006), 1159-1184.

[Gr] P. Grisvard: Elliptic problems in nonsmooth domains.Pitman, London, 1992.

[G] G. Grubb: Functional calculus of pseudodifferential boundary problems 2nd edn.. Birkhäuser, Boston, 1996.

[GK] G. Grubb and N.J. Kokholm: A global calculus of parameter-dependent pseudodifferential boundary problems in $L_{p}$ Sobolev spaces. Acta Math. 171 (1993), 1-100.

[K1] A.N. Kozhevnikov: Spectral problems for pseudodifferential systems elliptic in the DouglisNirenberg sense, and their applications. Math. USSR Sobornik 21 (1973), 63-90.

[K2] A.N. Kozhevnikov: Parameter-ellipticity for mixed order boundary problems. C. R. Acad. Sci. Paris Sér. I Math. 324 (1997), 1361-1366.

[K3] A.N. Kozhevnikov: Parameter-ellipticity for mixed order systems in the sense of Petrovskii. Commun. Appl. Anal. 5 (2001), 277-291.

[T] H. Triebel: Interpolation theory, function spaces, differential operators. North-Holland, Amsterdam, 1978.

[VL] M.I. Vishik and L. A. Lyusternik: Regular degeneration and boundary layer for linear differential equations with small parameter. Amer. Math. Soc. Transl.(2) 20 (1962), 239-264

[V] L.R. Volevich: Solvability of boundary value problems for general elliptic systems. Amer. Math. Soc. Transl.(2), 67 (1968), 182-225.

Department of Mathematics and Statistics, University of Konstanz, D-78457 KonSTANZ, GERMANY

E-mail address: robert.denk@uni-konstanz.de

School of Mathematics and Statistics, The University of New South Wales, UnSW SYDNeY, NSW 2052, Australia

E-mail address: ams11@maths.unsw.edu.au 